\title{
The surprisingly carbon-rich environment of the S-type star W AqI $\mathbf{I}^{\star \star \star}$
}

\author{
E. De Beck and H. Olofsson
}

\begin{abstract}
Department of Space, Earth and Environment, Chalmers University of Technology, Onsala Space Observatory, 43992 Onsala, Sweden e-mail: elvire.debeck@chalmers.se
\end{abstract}

Received 4 May 2020 / Accepted 3 July 2020

\begin{abstract}
Context. W Aql is an asymptotic giant branch (AGB) star with an atmospheric elemental abundance ratio $\mathrm{C} / \mathrm{O} \approx 0.98$. It has previously been reported to have circumstellar molecular abundances intermediate between those of M-type and C-type AGB stars, which respectively have $\mathrm{C} / \mathrm{O}<1$ and $\mathrm{C} / \mathrm{O}>1$. This intermediate status is considered typical for S-type stars, although our understanding of the chemical content of their circumstellar envelopes is currently rather limited.

Aims. We aim to assess the reported intermediate status of $\mathrm{W}$ Aql by analysing the line emission of molecules that have never before been observed towards this star.

Methods. We performed observations in the frequency range $159-268 \mathrm{GHz}$ with the SEPIA/B5 and PI230 instruments on the APEX telescope. We made abundance estimates through direct comparison to available spectra towards a number of well-studied AGB stars and based on rotational diagram analysis in the case of one molecule.

Results. From a compilation of our abundance estimates and those found in the literature for two M-type (R Dor, IK Tau), two S-type ( $\chi$ Cyg, W Aql), and two C-type stars (V Aql, IRC +10216), we conclude that the circumstellar environment of W Aql appears considerably closer to that of a C-type AGB star than to that of an M-type AGB star. In particular, we detect emission from $\mathrm{C}_{2} \mathrm{H}, \mathrm{SiC}_{2}$, $\mathrm{SiN}$, and $\mathrm{HC}_{3} \mathrm{~N}$, molecules previously only detected towards the circumstellar environment of C-type stars. This conclusion, based on the chemistry of the gaseous component of the circumstellar environment, is further supported by reports in the literature on the presence of atmospheric molecular bands and spectral features of dust species which are typical for C-type AGB stars. Although our observations mainly trace species in the outer regions of the circumstellar environment, our conclusion matches closely that based on recent chemical equilibrium models for the inner wind of S-type stars: the atmospheric and circumstellar chemistry of S-type stars likely resembles that of C-type AGB stars much more closely than that of M-type AGB stars.

Conclusions. Further observational investigation of the gaseous circumstellar chemistry of S-type stars is required to characterise its dependence on the atmospheric C/O. Non-equilibrium chemical models of the circumstellar environment of AGB stars need to address the particular class of S-type stars and the chemical variety that is induced by the range in atmospheric C/O.
\end{abstract}

Key words. stars: AGB and post-AGB - stars: mass-loss - astrochemistry - stars: individual: W Aql

\section{Introduction}

Evolved stars on the asymptotic giant branch (AGB) are typically classified according to the abundance ratio of carbon and oxygen atoms, $\mathrm{C} / \mathrm{O}$, in combination with a set of signatures pertaining to molecular bands. Strong molecular bands of titanium monoxide, TiO, are seen in the atmospheres of M-type AGB stars $(\mathrm{C} / \mathrm{O}<1)$; zirconium oxide, $\mathrm{ZrO}$, bands are seen for S-type stars $(\mathrm{C} / \mathrm{O} \approx 1)$; and bands of carbon compounds, such as $\mathrm{CN}$, are indicative of C-type (C/O > 1) stars (Habing \& Olofsson 2004).

The atmospheric content of S-type stars, and in particular the abundance ratio $\mathrm{TiO} / \mathrm{ZrO}$, is critically linked to the value of the $\mathrm{C} / \mathrm{O}$ abundance ratio (e.g. Smolders et al. 2012) and one can expect a similar link to exist for the contents of the circumstellar envelopes (CSEs) of these stars, built up by the stellar mass loss.

\footnotetext{
${ }^{\star}$ The FITS file containing the fully reduced spectrum presented in this paper is available at the CDS via anonymous ftp to cdsarc.u-strasbg.fr (130.79.128.5) or via http://cdsarc. u-strasbg.fr/viz-bin/cat/J/A+A/642/A20

$\star \star$ This publication is based on data acquired with the Atacama Pathfinder Experiment (APEX). APEX is a collaboration between the Max-Planck-Institut für Radioastronomie, the European Southern Observatory, and the Onsala Space Observatory.
}

Hony et al. (2009) and Smolders et al. (2010) showed that the dust around S-type stars can show a mixture of dust species typical for M-type and C-type stars, including silicates, $\mathrm{MgS}$, and possibly even polycyclic aromatic hydrocarbons (PAHs).

Chemical models of the gas in AGB CSEs reported in the literature focus primarily on M-type and C-type stars (e.g. Agúndez \& Cernicharo 2006; Agúndez et al. 2012; Cherchneff 2006, 2012; Cordiner \& Millar 2009; Gobrecht et al. 2016; Van de Sande et al. 2018; Willacy \& Millar 1997). Recently, Agúndez et al. (2020) presented chemical equilibrium models for S-type stars, but non-equilibrium chemical models for S-type stars with a similar coverage of molecular species are currently lacking. Additionally, the available chemical models assume high massloss rates $\left(\approx 10^{-5} M_{\odot} \mathrm{yr}^{-1}\right.$; most often using the M-type IK Tau and the C-type IRC +10216 as reference stars) and leave a clear gap in the description of the chemical content of CSEs of lowto intermediate-mass-loss rate stars $\left(10^{-8}-10^{-6} M_{\odot} \mathrm{yr}^{-1}\right)$.

Sample studies of the molecular content of the CSEs of S-type stars are currently limited to targeted observations of standard molecules, such as CO, $\mathrm{SiO}, \mathrm{SiS}, \mathrm{HCN}$, and CS (Danilovich et al. 2018; Ramstedt \& Olofsson 2014; Ramstedt et al. 2009; Schöier et al. 2013). Sample sizes vary from six to forty across these studies. The gas chemical content of the CSEs of the 


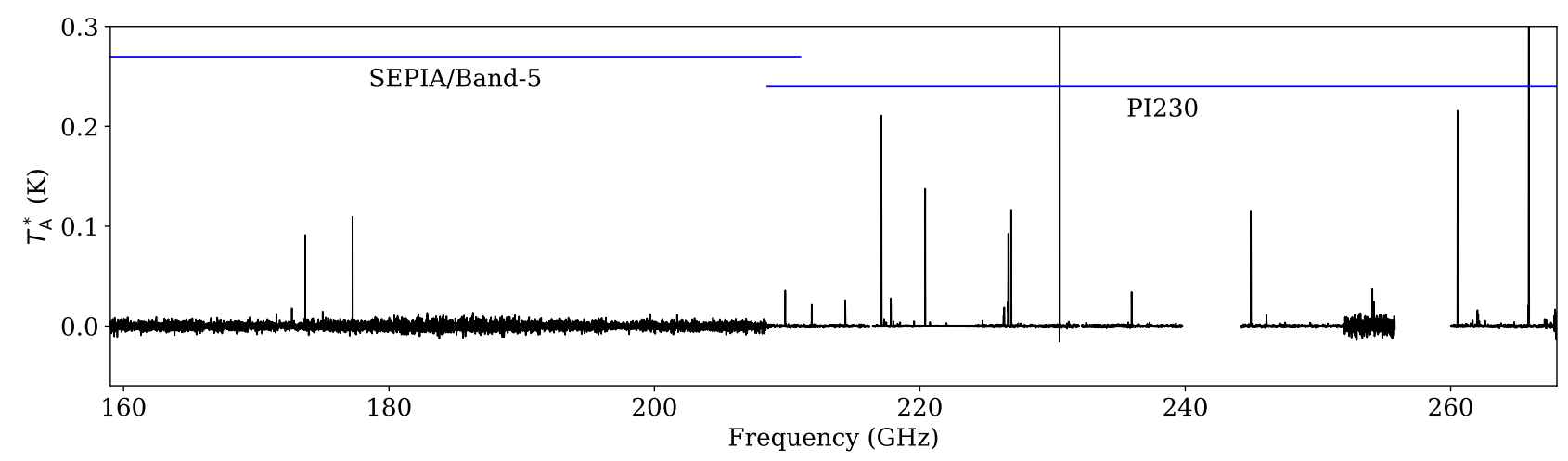

Fig. 1. APEX observations towards W Aql. The frequency ranges covered by the SEPIA/B5 and PI230 instruments are marked with blue horizontal lines.

S-type AGB stars W Aql and $\chi$ Cyg was studied in more detail by Decin et al. (2008), Schöier et al. (2011), Danilovich et al. (2014), and Brunner et al. (2018) using ALMA and Herschel observations, adding $\mathrm{H}_{2} \mathrm{O}, \mathrm{NH}_{3}$, and $\mathrm{CN}$ to the list of molecules detected towards S-type AGB stars. Unbiased observations of the CSEs of S-type stars, not targeting particular molecules but covering large bandwidths to also detect "unexpected" species, have not yet been presented in the literature.

We present observations at long wavelengths $(1-2 \mathrm{~mm})$ of molecular line emission from the CSE of the S-type AGB star W Aql. This star has a reported $\mathrm{C} / \mathrm{O} \approx 0.98$ (Keenan \& Boeshaar 1980; Danilovich et al. 2015) and shows a mix of M-type and Ctype signatures in its dust spectrum (Hony et al. 2009). W Aql is a Mira-type variable with a pulsation period of 490 days (Alfonso-Garzón et al. 2012), is located at an estimated distance of $395 \mathrm{pc}$ (Danilovich et al. 2014, and references therein), and has a mass-loss rate of $3 \times 10^{-6} M_{\odot} \mathrm{yr}^{-1}$ (Ramstedt et al. 2017).

We discuss the molecular footprint of W Aql's CSE, how this compares to what we know about its atmosphere and dust, and put this in relation to what is known about CSEs of "prototypical" M-type (R Dor and IK Tau) and C-type (IRC +10 216, V Aql) stars.

\section{Observations}

In the framework of a broader project in which the effect of AGB wind density on the circumstellar chemistry is studied, we carried out a spectral survey of $\mathrm{W}$ Aql in the range 159-211 GHz using Band 5 of the Swedish-ESO PI Instrument (SEPIA; Billade et al. 2012; Belitsky et al. 2018) on the Atacama Pathfinder Experiment telescope (APEX; Güsten et al. 2006). The observations were carried out on 9 May 2017 in very good weather, at $0.8-1.5 \mathrm{~mm}$ of precipitable water vapour (PWV). We obtained follow-up observations using the APEX/PI230 instrument $^{1}$ on 17 and 21 May, 21 and 22 August, and 21 October 2018 with a PWV in the range $0.7-1.0 \mathrm{~mm}$, and on $2,3,7$, and 11 July 2019 with a PWV in the range $0.9-5.8 \mathrm{~mm}$.

All observations were carried out in wobbler-switching mode using the standard $50^{\prime \prime}$ beam throw. The size of the main beam is $30-39^{\prime \prime}$ at $159-211 \mathrm{GHz}$ (SEPIA/B5) and $27-30^{\prime \prime}$ at 209-236 GHz and 23-25" at 244-272 GHz (PI230).

We process the fully calibrated data with the GILDAS/ CLASS $^{2}$ package. After removing bad channels, which are

1 PI230 is a collaboration between the European Southern Observatory (ESO) and the Max-Planck-Institut für Radioastronomie (MPIfR).

2 http://www.iram.fr/IRAMFR/GILDAS/
Table 1. Noise characteristics.

\begin{tabular}{lccc}
\hline Instrument & $\begin{array}{c}\text { Frequency range } \\
(\mathrm{GHz})\end{array}$ & $\begin{array}{c}\mathrm{Rms} \\
(\mathrm{mK})\end{array}$ & $\begin{array}{c}\Delta v \\
(\mathrm{MHz})\end{array}$ \\
\hline SEPIA/B5 & $159.0-178.0$ & 3.5 & 3.2 \\
& $178.0-188.0$ & 5.0 & 3.2 \\
PI230 & $188.0-208.4$ & 3.5 & 3.2 \\
& $208.4-252.0$ & 0.5 & 4.3 \\
& $252.0-255.8$ & 5.5 & 5.1 \\
& $260.0-267.8$ & 0.6 & 5.4 \\
\hline
\end{tabular}

Notes. We list approximate rms noise across different frequency intervals in our data for frequency resolution $\Delta v$.

typically present at $<50 \mathrm{MHz}$ from the band edges, and masking relevant spectral features we remove a first-degree polynomial baseline from the spectra before averaging. We show the resulting spectra in $T_{\mathrm{A}}^{*}$, the antenna temperature corrected for spillover and atmospheric losses, throughout the paper. A snapshot overview is shown in Fig. 1; for detailed spectra we refer to Figs. A.1 (SEPIA/B5) and A.2 (PI230). We refer to Appendix A for a brief discussion on the occurrence of a small number of features in the spectra that originate from sideband contamination.

Noise characteristics vary significantly throughout the covered spectral range and are summarised in Table 1. These values are approximate, as the rms noise can vary somewhat depending on the exact location in the spectrum as a consequence of different observing conditions (see above). The increase in rms noise in the SEPIA//B5 range is caused by the interference of atmospheric water around $183 \mathrm{GHz}$. The high noise in the range $252.0-255.8 \mathrm{GHz}$ is a consequence of the short integration time at this frequency, connected to tuning problems. We include the data as they include several detected lines. The frequency resolution at which the rms noise values in Table 1 are measured translates to a velocity resolution in the range $5.5-6.5 \mathrm{~km} \mathrm{~s}^{-1}$, allowing for detection of circumstellar spectral lines towards W Aql (see Sect. 3).

We adopt a systemic velocity $v_{\text {sys }}=-22.8 \mathrm{~km} \mathrm{~s}^{-1}$ for $\mathrm{W}$ Aql and all velocities $v$ reported here are relative to this value within the local standard of rest (LSR) framework, that is $v=v_{\mathrm{LSR}}-v_{\text {sys }}$.

\section{Results}

We show the entire data set in Figs. A.1 (SEPIA/B5) and A.2 (PI230) and provide an overview of all measured emission 
Table 2. Molecules identified in our survey towards W Aql.

\begin{tabular}{|c|c|c|}
\hline Molecule & $\begin{array}{l}\text { Number } \\
\text { of lines }\end{array}$ & Line numbers \\
\hline${ }^{13} \mathrm{CN}^{(\ddagger)}$ & 3 & $24,25,26$ \\
\hline${ }^{13} \mathrm{CO}$ & 1 & 33 \\
\hline${ }^{13} \mathrm{CS} \stackrel{(\ddagger)}{(1)}$ & 1 & 43 \\
\hline${ }^{29} \mathrm{SiO}$ & 2 & 4,22 \\
\hline${ }^{29} \mathrm{SiS}^{(\ddagger)}$ & 3 & $21,60,83$ \\
\hline${ }^{30} \mathrm{SiO}^{(\ddagger)}$ & 2 & 17,63 \\
\hline${ }^{30} \mathrm{SiO}^{(\dagger)}(\ddagger)$ & 1 & 3 \\
\hline${ }^{30} \mathrm{SiS}$ & 3 & $16,41,76$ \\
\hline${ }^{30} \mathrm{SiS}^{(\dagger)}$ & 1 & 53 \\
\hline $\mathrm{C}^{17} \mathrm{O}$ & 1 & 36 \\
\hline $\mathrm{C}^{18} \mathrm{O}$ & 1 & 31 \\
\hline $\mathrm{C}^{34} \mathrm{~S}^{(\dagger)}(\dagger)$ & 1 & 12 \\
\hline $\mathrm{C}_{2} \mathrm{H}^{(\ddagger)}$ & 2 & 72,73 \\
\hline $\mathrm{C}_{2} \mathrm{H}^{(\dagger)}(\ddagger)$ & 3 & $8,9,75$ \\
\hline $\mathrm{CN}$ & 3 & $37,38,39$ \\
\hline $\mathrm{CO}$ & 1 & 42 \\
\hline $\mathrm{CS}$ & 1 & 52 \\
\hline $\mathrm{H}^{13} \mathrm{CN}$ & 1 & 5 \\
\hline $\mathrm{HC}_{3} \mathrm{~N}^{(\dagger)}(\ddagger)$ & 7 & $6,14,29,40,48,54,77$ \\
\hline $\mathrm{HCN}$ & 2 & 10,80 \\
\hline $\mathrm{HCN}, v_{2}=1$ & 2 & 79,82 \\
\hline $\mathrm{HCO}^{+}(\dagger)(\ddagger)$ & 2 & 11,84 \\
\hline $\mathrm{HN}^{13} \mathrm{C}^{(\ddagger)}$ & 1 & 68 \\
\hline $\mathrm{NaCl}^{(\dagger)(\ddagger)}$ & 2 & 57,65 \\
\hline $\mathrm{Si}^{17} \mathrm{O}^{(\ddagger)}$ & 1 & 61 \\
\hline $\mathrm{Si}^{34} \mathrm{~S}^{(\ddagger)}$ & 3 & $18,56,78$ \\
\hline $\mathrm{SiC}_{2}(\ddagger)$ & 13 & $\begin{array}{l}19,20,34,35,44,45,46,49 \\
50,58,67,69,71\end{array}$ \\
\hline $\mathrm{SiC}_{2}(\dagger)(\ddagger)$ & 1 & 64 \\
\hline $\mathrm{SiN}^{(\ddagger)}$ & 4 & $28,30,70,74$ \\
\hline $\mathrm{SiO}$ & 3 & $7,23,66$ \\
\hline $\mathrm{SiS}$ & 4 & $13,27,47,62$ \\
\hline $\mathrm{SiS}^{(\dagger)}$ & 1 & 2 \\
\hline $\mathrm{u}$ & 2 & 32,59 \\
\hline
\end{tabular}

Notes. Line numbers listed in Col. 3 correspond to those in Col. 1 of Table A.2. We mark both new and tentative detections for clarity. ${ }^{(\dagger)}$ Tentative detection or identification. ${ }^{(\dot{*})}$ Species not previously reported towards W Aql.

features and their main characteristics in Table A.2. We summarise the identified molecules in Table 2.

We retain spectral features if the signal in at least three out of six consecutive channels of width $\Delta v$ exceeds $3 \sigma$ with $\sigma$ and $\Delta v$ equal to the rms and frequency resolution listed in Table 1. This allows us to recover spectral lines over a total velocity interval of roughly two times the terminal velocity of $\mathrm{W}$ Aql, $v_{\infty} \approx 16.5 \mathrm{~km} \mathrm{~s}^{-1}$ (Danilovich et al. 2014).

All line identifications stem from the Cologne Database for Molecular Spectroscopy ${ }^{3}$ (CDMS; Müller et al. 2001, 2005). We also used the catalogue for molecular line spectroscopy hosted by the Jet Propulsion Laboratory ${ }^{4}$ (JPL; Pickett et al. 1998) and Splatalogue ${ }^{5}$ as additional resources. Some features are reported

\footnotetext{
3 https://cdms.astro.uni-koeln.de/

4 http://spec.jpl.nasa.gov/

5 http://www.cv.nrao.edu/php/splat/advanced.php
}

as tentative detections although they may not strictly fulfill the above criterion. This is the case if we expect their presence based on other transitions or hyperfine components of the same transition of a given molecule and we find a plausible signal at a lower signal-to-noise ratio $(\mathrm{S} / \mathrm{N})$. Moreover, the combination of tentative detections of multiple transitions of a given molecule can lead to a firm detection of that molecule, as we discuss in Sect. 3.1.2 in the case of $\mathrm{HC}_{3} \mathrm{~N}$.

In Sect. 3.1 we summarise which molecules we identify in our observations, how our results compare to earlier reports on CSEs of M-, S-, and C-type AGB stars in general, and on the CSE of W Aql in particular. In Sect. 3.2 we shortly discuss the detection or non-detection of different isotopologues for some of the main elements.

\subsection{Molecular inventory}

We detect emission from transitions of $\mathrm{CO}, \mathrm{SiO}, \mathrm{HCN}, \mathrm{CS}, \mathrm{SiS}$, and $\mathrm{CN}$ as expected for the CSE of an S-type AGB star and consistent with previous reports for W Aql (e.g. Brunner et al. 2018; Danilovich et al. 2014, 2018; Schöier et al. 2011). We additionally detect emission from species considered to be typical for, or until now only detected in, the CSEs of carbon-rich AGB stars: $\mathrm{SiC}_{2}, \mathrm{SiN}, \mathrm{C}_{2} \mathrm{H}$, and the cyanopolyyne $\mathrm{HC}_{3} \mathrm{~N}$. We give an overview of these detections and provide some first abundance estimates to relate our findings to those made for M-type and C-type CSEs. We briefly address some tentative detections and unidentified emission features.

\subsubsection{Previously detected: $\mathrm{CO}, \mathrm{SiO}, \mathrm{HCN}, \mathrm{CS}, \mathrm{SiS}, \mathrm{CN}$}

$\mathrm{CO}, \mathrm{SiO}, \mathrm{HCN}$, and $\mathrm{CS}$ are expected to form in relatively high abundances in the inner winds of all chemical types of AGB stars (Cherchneff 2006; Agúndez et al. 2020). SiS emission is also commonly detected towards CSEs around all chemical types of AGB stars (Schöier et al. 2013). We refer to the literature for detailed discussions on these molecules in the CSE of W Aql: Ramstedt \& Olofsson (2014) and Danilovich et al. (2014) present radiative-transfer models of $\mathrm{CO}$ emission and Brunner et al. (2018) present models of $\mathrm{SiO}, \mathrm{HCN}, \mathrm{CS}$, and SiS.

CO. We detect emission from ${ }^{12} \mathrm{CO}(J=2-1)$ and ${ }^{13} \mathrm{CO}(J=2-1)$ (see Fig. B.1), in agreement with the lines published by De Beck et al. (2010) and Ramstedt \& Olofsson (2014) and with the calibration spectra made available by the APEX observatory ${ }^{6}$.

SiO. We detect three emission lines from the main $\mathrm{SiO}$ isotopologue $(J=4-3,5-4,6-5)$ and one from $\mathrm{Si}^{17} \mathrm{O}(J=6-5)$ at $250.7 \mathrm{GHz}$. We do not detect emission from $\mathrm{Si}^{18} \mathrm{O}$, in line with the intensity of the $\mathrm{Si}^{17} \mathrm{O}$ emission and the sensitivity reached in the spectra covering the relevant transitions of $\mathrm{Si}^{18} \mathrm{O}$ assuming ${ }^{17} \mathrm{O} /{ }^{18} \mathrm{O}=1.2$ (De Nutte et al. 2017). We detect the ${ }^{29} \mathrm{SiO}(J=4-3,5-4)$ and ${ }^{30} \mathrm{SiO}(J=4-3,5-4,6-5)$ lines. An overview is shown in Fig. B.3.

$H C N$. We detect emission from $\mathrm{HCN}$ in the vibrational ground state $v=0 \quad(J=2-1,3-2)$, from both $l$-type doubling components of $J=3-2$ in the excited bending mode $v_{2}=1$, and from $\mathrm{H}^{13} \mathrm{CN}(J=2-1)$; see Fig. B.2.

CS. We detect emission from $\mathrm{CS}(J=5-4)$ at $244.9 \mathrm{GHz}$ with a peak intensity of $115 \mathrm{mK}(\approx 4.5 \mathrm{Jy})$; see Fig. B.5. We do

\footnotetext{
6 Spectra can be retrieved from http://www.apex-telescope. org/heterodyne/shfi/calibration/database
} 

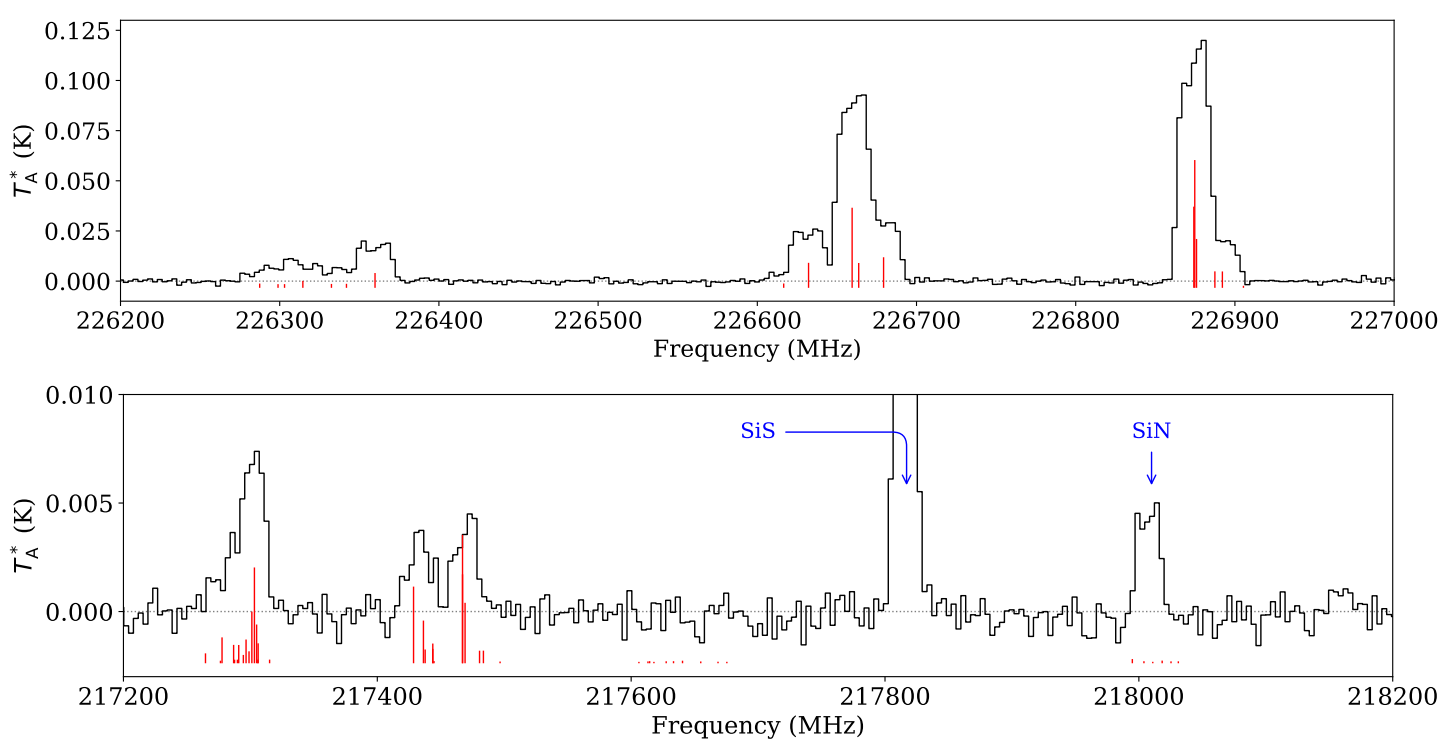

Fig. 2. $\mathrm{CN}$ emission; top: ${ }^{12} \mathrm{CN}$, bottom: ${ }^{13} \mathrm{CN}$. Vertical red lines indicate the position and relative strength of the hyperfine structure components of the rotational transition $2-1$.

not detect emission from $\mathrm{CS}(J=4-3)$ at $196.0 \mathrm{GHz}$ and can set an upper limit to the peak intensity of $12 \mathrm{mK}(\approx 0.5 \mathrm{Jy})$. The $J=5-4$ intensity is roughly in line with the observed intensities of the $J=3-2,6-5,7-6$ lines reported by Brunner et al. (2018), whereas the $J=4-3$ upper limit lies far below the expectations. Cernicharo et al. (2014) report that low-J CS emission lines show only small variability over time in the case of IRC +10216 , so we believe that time variability in the line excitation does not explain the observed discrepancy. Since we do not perform radiative transfer modelling for this paper, we wish to not hypothesise on the reason for the non-detection of the $J=4-3$ line.

We detect emission from ${ }^{13} \mathrm{CS}(J=5-4)$ at $231.2 \mathrm{GHz}$ at a peak intensity of $\approx 5 \mathrm{mK}$ and can set an upper limit of $7 \mathrm{mK}$ to the peak intensity of ${ }^{13} \mathrm{CS}(J=4-3)$ at $185.0 \mathrm{GHz}$. We also detect emission from $\mathrm{C}^{34} \mathrm{~S}(J=4-3)$ at $192.8 \mathrm{GHz}$.

SiS. We detect emission from the SiS transitions $J=9-8$, $11-10,12-11,13-12,14-13$ but do not detect $\operatorname{SiS}(J=10-9)$ at $181.5 \mathrm{GHz}$ owing to the higher noise in this region of the SEPIA/B5 spectrum; see Figs. A.1 and A.2 and Table 1. We also detect emission from the isotopologues ${ }^{29} \mathrm{SiS}(J=12-11$, $14-13,15-14),{ }^{30} \mathrm{SiS}(12-11,13-12,14-13,15-14)$, and $\mathrm{Si}^{34} \mathrm{~S}$ $(J=12-11,14-13,15-14)$; see Fig. B.4.

$C N$. We detect emission over the full hyperfine structure of $\mathrm{CN} N=2-1$ between 226.2 and $227.0 \mathrm{GHz}$ and of ${ }^{13} \mathrm{CN}$ $N=2-1$ between 217.2 and $217.5 \mathrm{GHz}$ (Fig. 2). Whereas we report the first detection of ${ }^{13} \mathrm{CN}$ towards $\mathrm{W}$ Aql, emission from $\mathrm{CN}(N=1-0,2-1)$ has been detected before with the IRAM30m telescope (Bachiller et al. 1997), but at lower S/N than attained in the APEX data. Using Kelvin-to-Jansky conversion factors of $38 \mathrm{Jy} \mathrm{K}^{-1}$ for SEPIA/B5 (De Beck \& Olofsson 2018, and references therein) and $7.2 \mathrm{Jy} \mathrm{K}^{-1}$ for the IRAM observations, in combination with a main-beam efficiency $\eta_{\mathrm{MB}}$ of 0.40 for the IRAM observations (Bachiller et al. 1997), we find that the peak fluxes for the strongest $N=2-1$ features in Fig. 2 reach $\approx 3.8-4.6 \mathrm{Jy}$, whereas those measured by Bachiller et al. (1997) reach values of only $\approx 1.2-1.4 \mathrm{Jy}$. Without performing any further modelling, we hypothesise here that stellar variability might be responsible for this factor of approximately three difference in intensity, based on the conclusion by Bachiller et al. (1997) that radiative pumping through optical and near-infrared (NIR) bands likely dominates the $\mathrm{CN}$ excitation (calibration and/or pointing errors in the IRAM data cannot be excluded). The radiativetransfer models presented by Danilovich et al. (2014) for W Aql show an increase of $\mathrm{CN}$ at the photodissociation radius of $\mathrm{HCN}$, in agreement with $\mathrm{CN}$ being produced as a photodissociation product of HCN. However, those models are only constrained by the $N=1-0$ and $N=2-1$ transitions and do not necessarily trace regions closer to the star or any possible variability of the emission over time.

\subsubsection{New detections: $\mathrm{SiC}_{2}, \mathrm{SiN}, \mathrm{C}_{2} \mathrm{H}, \mathrm{HC}_{3} \mathrm{~N}$}

$\mathrm{SiC}_{2}$. We detect fourteen emission lines pertaining to $\mathrm{SiC}_{2}$ (Fig. 3). We measure similar intensities for, for example, the $9_{4,6}-8_{4,5}(213.2 \mathrm{GHz})$ and $9_{4,5}-8_{4,4}(213.3 \mathrm{GHz})$ lines and for the $11_{4,8}-10_{4,7}(261.2 \mathrm{GHz})$ and $11_{4,7}-10_{4,6}(261.5 \mathrm{GHz})$ lines. This is in line with the two transitions in each pair having almost identical intrinsic strengths and upper-level energies. The pairwise match in intensities confirms the identification of $\mathrm{SiC}_{2}$ in our spectra.

We construct a rotational temperature diagram for $\mathrm{SiC}_{2}$ based on the measured emission of 12 out of 14 detected transitions (Fig. 4). We leave out two transitions: $11_{2,10}-10_{2,9}$ at $254.981 \mathrm{GHz}$ because of the large uncertainty owing to very high rms noise in this spectral region and $12_{0,12}-11_{0,11}$ at $261.990 \mathrm{GHz}$ which is blended with a much stronger signal from $\mathrm{C}_{2} \mathrm{H}$. We assume equal integrated intensity for the fully blended emission of the $10_{6,5}-9_{6,4}$ and $10_{6,4}-9_{6,3}$ transitions as they have identical transition probabilities (Einstein- $A$ coefficients). Under the assumption of optically thin emission excited under LTE conditions, we find a rotational temperature $T_{\text {rot }}=76 \pm 28 \mathrm{~K}$. We find a source-averaged column density of $(2.1 \pm 0.9) \times 10^{14} \mathrm{~cm}^{-2}$ and an abundance $\mathrm{SiC}_{2} / \mathrm{H}_{2} \approx 5 \times 10^{-7}$ when assuming that the emission arises in a spherical shell located at $1-2^{\prime \prime}$ in radius, that is, at a distance of $\sim 400-800 \mathrm{AU}$ from the star. The assumption of a peak in the $\mathrm{SiC}_{2}$ abundance at roughly $600 \mathrm{AU}$ from the star is based on (1) the fact that one rotational temperature seems to represent all detected emission in our data reasonably 

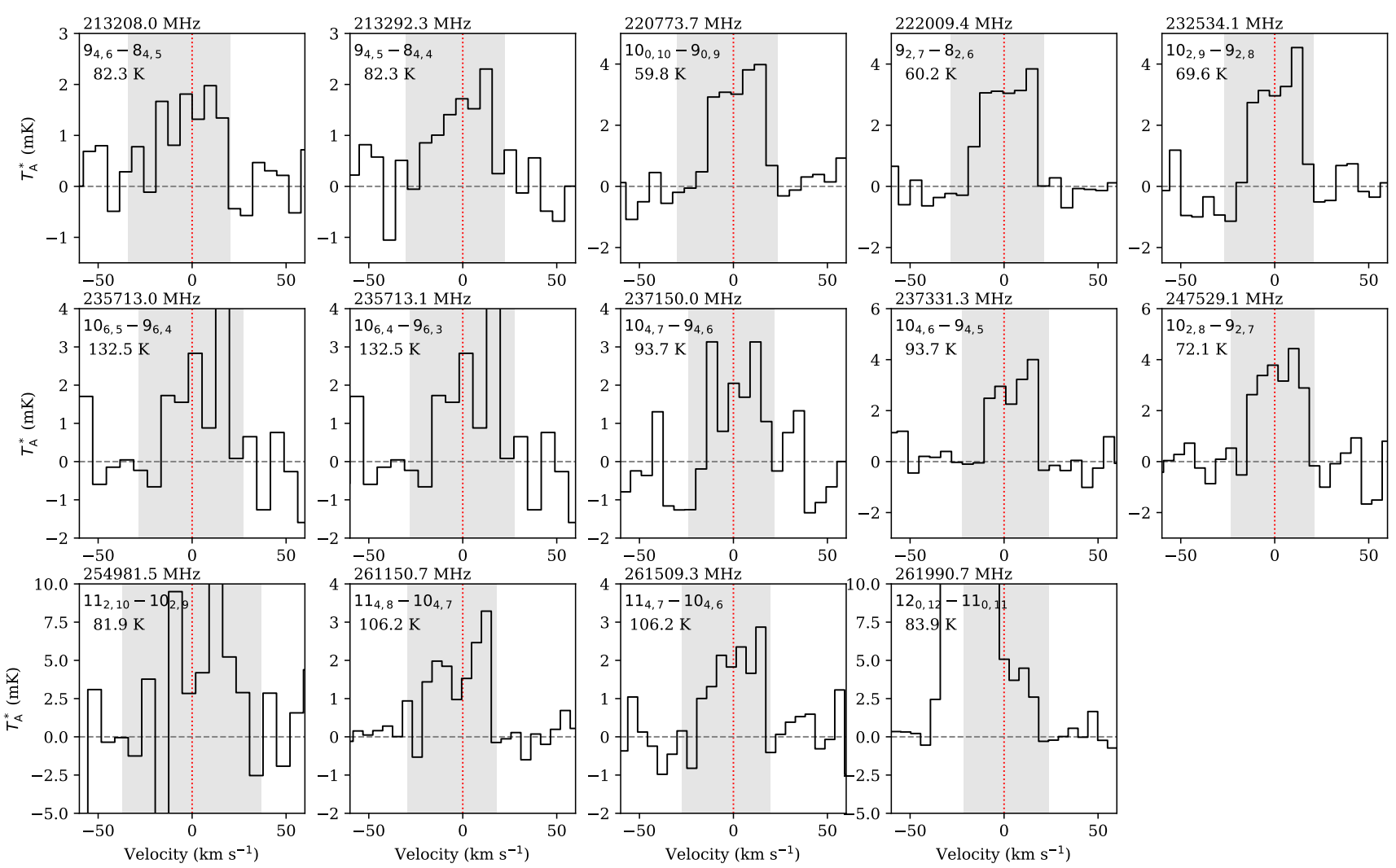

Fig. 3. $\mathrm{SiC}_{2}$ emission. Shaded areas indicate the part of the spectrum integrated to obtain the intensities used to construct the rotational diagram in Fig. 4, and vertical red lines correspond to the rest frequency, centred to a velocity of $0 \mathrm{~km} \mathrm{~s}^{-1}$. We give the rest frequency, quantum numbers, and upper level energy for each transition. We note that (i) the rest frequencies of the $10_{6,5}-9_{6,4}$ and $10_{6,4}-9_{6,3}$ lines at $235.7 \mathrm{GHz}$ differ by only $0.1 \mathrm{MHz}$ and (ii) the emission from the $12_{0,12}-11_{0,11}$ transition at $261.991 \mathrm{GHz}$ (last panel) is blended with intense $\mathrm{C}_{2} \mathrm{H}$ emission (Fig. 5) and is therefore omitted from the rotational diagram analysis, as is the $11_{2,10}-10_{2,9}$ transition which is tentatively detected in a region of high rms noise.

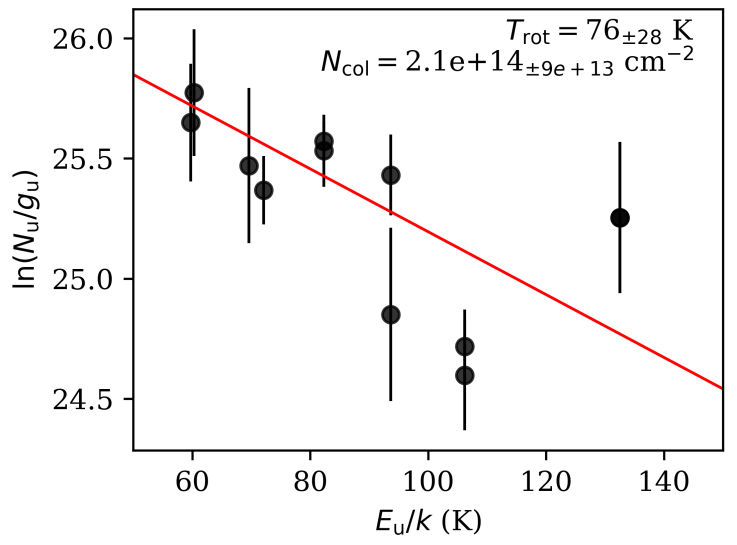

Fig. 4. Rotational temperature diagram for $\mathrm{SiC}_{2}$.

well, (2) the location in the CSE where the derived rotational temperature is reached according to the temperature profile presented by Danilovich et al. (2014) is roughly $600 \mathrm{AU}$, and (3) the fact that $\mathrm{SiC}_{2}$ is also observed in a shell around IRC +10 216 (Velilla-Prieto et al. 2019). However, the $\mathrm{SiC}_{2}$ emission towards IRC +10216 is located in a shell at $\sim 14-16^{\prime \prime}$ from the central star, which is at roughly three times larger physical separation from the central star compared to what we assume for W Aql. Assuming the equivalent spatial distribution for $\mathrm{SiC}_{2}$ in $\mathrm{W}$ Aql's $\mathrm{CSE}$, we obtain a slightly higher abundance $\mathrm{SiC}_{2} / \mathrm{H}_{2} \approx 8 \times 10^{-7}$, which is still well within the uncertainties of our simple approach here ${ }^{7}$. The difference in spatial occurrence of $\mathrm{SiC}_{2}$ could be a consequence of the roughly three times higher mass-loss rate of IRC +10216 , and hence the correspondingly higher densities in the CSE. Comparing our estimated abundance to the results presented by Massalkhi et al. (2018, see their Fig. 6) for a sample of $25 \mathrm{C}$-rich envelopes, we find that the combination of the $\mathrm{SiC}_{2}$ abundance and wind density $\dot{M} / v_{\infty}$ of $\mathrm{W}$ Aql fits well within the distribution of the C-rich envelopes, with W Aql's $\mathrm{SiC}_{2}$ abundance being on the lower end of the presented sample. Accounting for the uncertainties inherent to our approach, we conclude that this result indicates a possible similarity in chemistry between the CSE of the S-type AGB star W Aql and C-type AGB stars.

SiN. We detect emission from the strongest components of the $\operatorname{SiN}(N=5-4)$ transition at 218.01 and $218.51 \mathrm{GHz}$ and of $N=6-5$ at 261.65 and $262.15 \mathrm{GHz}$ (Figs. 5 and B.6; these components have $\Delta F=\Delta J$ ). Assuming similar line strengths, the non-detection of the $N=4-3$ lines at 174.36 and $174.86 \mathrm{GHz}$ is in line with the much higher rms-noise in that region. The hyperfine structure of the SiN transition is not resolved and has a very limited contribution of only about $1 \mathrm{MHz}$ to the broadening of the overall profile.

\footnotetext{
7 The uncertainties on the abundance estimate stem from multiple simplifying assumptions, including optically thin emission, LTE excitation, and the match between rotational temperature and excitation temperature. The uncertainties therefore amount to more than the numerical uncertainties quoted for $T_{\text {rot }}$ and $N_{\text {col }}$, which are based only on uncertainties introduced by the data quality.
} 


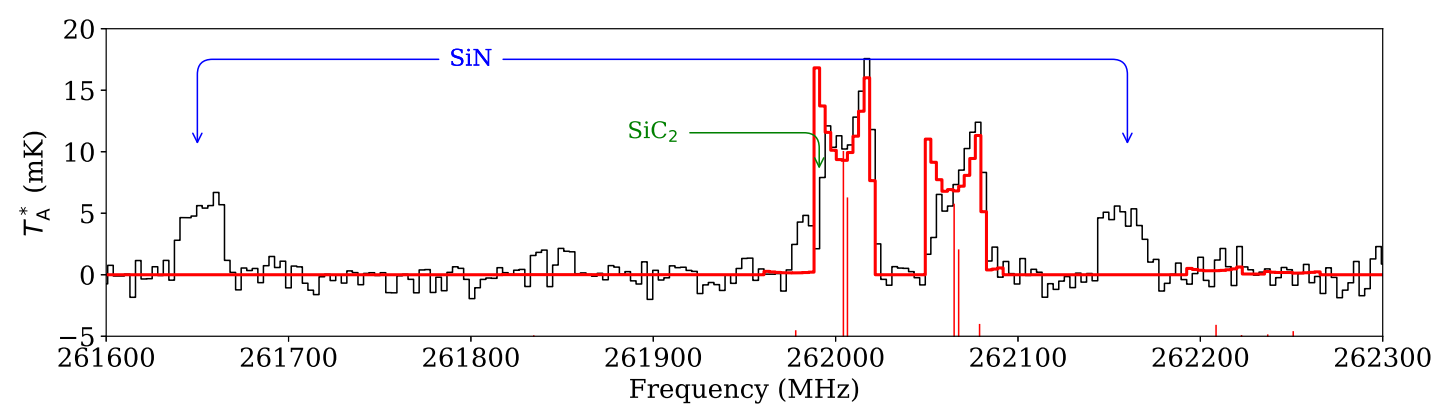

Fig. 5. $\mathrm{C}_{2} \mathrm{H}$ and SiN emission. High-signal-to-noise ratio detection of $\mathrm{C}_{2} \mathrm{H}(3-2)$ in the PI230 data, plotted at $3.0 \mathrm{MHz}\left(\approx 3.5 \mathrm{~km} \mathrm{~s}{ }^{-1}\right)$ spectral resolution. Vertical red lines indicate the position and relative strength of the hyperfine structure components of the rotational transition of $\mathrm{C}_{2} \mathrm{H}$. In red, we overplot a synthetic spectrum of $\mathrm{C}_{2} \mathrm{H}(3-2)$ using a "shell" profile as described in the GILDAS/CLASS manual, fitted to the data by eye, using the intrinsic component strengths. In blue we indicate the location of the strongest components of the $\operatorname{SiN}(N=6-5)$ transition; see Fig. B.6 for all detected $\mathrm{SiN}$ emission. We mark the rest frequency of $\mathrm{SiC}_{2}\left(12_{0,12}-11_{0,11}\right)$ in green.

We can directly compare our APEX observations of $\operatorname{SiN}(N=6-5)$ towards $\mathrm{W}$ Aql to the NRAO $12 \mathrm{~m}$ telescope observations of $\mathrm{SiN}(N=6-5)$ towards IRC +10216 (Turner 1992). Scaling the emission for mass-loss rate and distance (W Aql: $\dot{M}=3 \times 10^{-6} M_{\odot} \mathrm{yr}^{-1}, d=395 \mathrm{pc} ; \mathrm{IRC}+10216: \dot{M}=2 \times$ $10^{-5} M_{\odot} \mathrm{yr}^{-1}, d=130 \mathrm{pc}$; Brunner et al. 2018; Agúndez et al. 2012; Men'shchikov et al. 2001) we find that the SiN emission in $\mathrm{W}$ Aql is roughly five times as bright as the $\mathrm{SiN}$ emission in IRC +10216 (for $N=6-5$ ). Assuming optically thin emission and the same excitation conditions for $\mathrm{SiN}$ in both CSEs, this would imply $\left(\mathrm{SiN} / \mathrm{H}_{2}\right)_{\mathrm{W} \text { Aql }} \approx 5 \times\left(\mathrm{SiN} / \mathrm{H}_{2}\right)_{\mathrm{IRC}+10216}=4 \times 10^{-8}$ (Agúndez 2009).

$\mathrm{SiN}$ and $\mathrm{SiC}_{2}$ have both been detected towards the CSEs of C-type stars, but we are not aware of any reported detections of either molecule towards S-type or M-type AGB stars. Based on the angular resolution of 24-29" obtained in the APEX observations of $\mathrm{SiC}_{2}$ and $\mathrm{SiN}$ we cannot determine whether these molecules are present in the extended CSE of W Aql or only close to the star, but from the width of the line profiles we find that both the $\mathrm{SiC}_{2}$ and $\mathrm{SiN}$ emitting gas reach the terminal velocity of the wind, $v_{\infty} \approx 16.5 \mathrm{~km} \mathrm{~s}^{-1}$ (Danilovich et al. 2014), suggesting that both molecules are present beyond the wind-acceleration zone. Furthermore, in the case of $\mathrm{SiC}_{2}$, we determine a single rotational temperature low enough to be indicative of the presence of the molecule in the outer parts of the CSE (Danilovich et al. 2014, see their Figs. 3 and 4).

$\mathrm{C}_{2} \mathrm{H}$. We tentatively detect emission from $\mathrm{C}_{2} \mathrm{H}$ in the $N=2-1$ transition at $\sim 174.7 \mathrm{GHz}$, and detect emission at high signal-to-noise ratio for $N=3-2$ at $\sim 262.0 \mathrm{GHz}$ (Fig. 5). We produce a synthetic spectrum for the $N=3-2$ transition assuming a "shell" profile for each hyperfine component, as described in the GILDAS/CLASS manual, and using the tabulated intrinsic relative strengths and fit the sum of them to our data by eye (see Fig. 5). We find that the measured relative strengths of the hyperfine structure components agree very well with the intrinsic relative strengths tabulated in the CDMS (Müller et al. 2000).

The line profiles of the doublet components of $\mathrm{C}_{2} \mathrm{H}(N=3-2)$ are double-peaked, but we note that the peaks corresponding to the extreme velocities of the doublet components are not equal in strength, the blueshifted peaks being much stronger than the redshifted peaks in both components. The strong difference between the blue and red sides of the profiles hints at an asymmetry in the CSE with more $\mathrm{C}_{2} \mathrm{H}$ gas excited in the part of the CSE moving towards the observer. We cannot assess whether this would be caused by differences in excitation or in abundance distribution. Both of these could be a consequence of, for example, clumpiness in the CSE.

We also note that roughly the opposite is true in the $\mathrm{C}_{2} \mathrm{H}$ spectra towards IRC +10216 , where the redshifted peaks are stronger than the blueshifted peaks in the transitions $1-0, \ldots, 7-6$ presented by De Beck et al. (2012). In the case of IRC +10216 , the difference is likely a consequence of opticaldepth effects, where only the outermost and therefore colder $\mathrm{C}_{2} \mathrm{H}$ gas is visible in the front of the CSE, whereas we only see the emission from the warmer, inner $\mathrm{C}_{2} \mathrm{H}$ gas from the part of the CSE that moves away from the observer. In the case of IRC +10216 , we know that the $\mathrm{C}_{2} \mathrm{H}$ emission is spread over a thin, circular shell around the star. In the case of $\mathrm{W}$ Aql we lack direct information on the spatial distribution of the excited $\mathrm{C}_{2} \mathrm{H}$ gas, but the double-peaked spectrum suggests emission from a shell.

Scaling the measured $\mathrm{C}_{2} \mathrm{H}$ line emission for IRC +10216 to what one could expect at the distance of $\mathrm{W}$ Aql and assuming for simplicity that the emission is excited in a similar shelllike region around the star and that the line intensity scales linearly with $\dot{M}$, we expect the strongest components to peak at $\sim 10-15 \mathrm{mK}$ for $N=2-1$ and $N=3-2$ in our APEX data. Since the observed lines peak at $\sim 5-15 \mathrm{mK}$, we suggest that the abundance of $\mathrm{C}_{2} \mathrm{H}$ in the CSE of $\mathrm{W}$ Aql is roughly a factor of two lower than in the CSE of IRC +10216 , that is $\left(\mathrm{C}_{2} \mathrm{H} / \mathrm{H}_{2}\right)_{\mathrm{W} \text { Aql }} \approx 0.5 \times\left(\mathrm{C}_{2} \mathrm{H} / \mathrm{H}_{2}\right)_{\mathrm{IRC}+10216}=1 \times 10^{-5}(\mathrm{De}$ Beck et al. 2012). Cernicharo et al. (2014) note that the strength of the $\mathrm{C}_{2} \mathrm{H}$ emission in IRC +10216 is highly variable with time as a consequence of the strong effect of radiative pumping in the molecular excitation and the changes in the stellar radiation field owing to pulsations. However, since we consider here a low- $N$ rotational transition, this variability is likely limited to within the observational uncertainties (see their Fig. 2). We do not detect any emission from the longer-chain radicals $\mathrm{C}_{4} \mathrm{H}$ or $\mathrm{C}_{6} \mathrm{H}$ at the current sensitivity.

$\mathrm{HC}_{3} \mathrm{~N}$. We tentatively detect emission in multiple rotational transitions from $\mathrm{HC}_{3} \mathrm{~N}$. Figure 6 shows an overview of the spectral ranges where rotational transitions of $\mathrm{HC}_{3} \mathrm{~N}$ are covered by our data. Some transitions are not detected (marked ND) and some are tentatively detected (marked $\mathrm{T}$ ). We also show the result of stacking the data, aligned in velocity space, weighted with the ratio of the statistical weights $2 J+1$ of the upper levels of the respective transitions $J \rightarrow J-1$ and the rms noise in the spectrum close to the transition's rest frequency. We performed this stacking procedure for three sets of lines; we obtain $S / N>5$ 

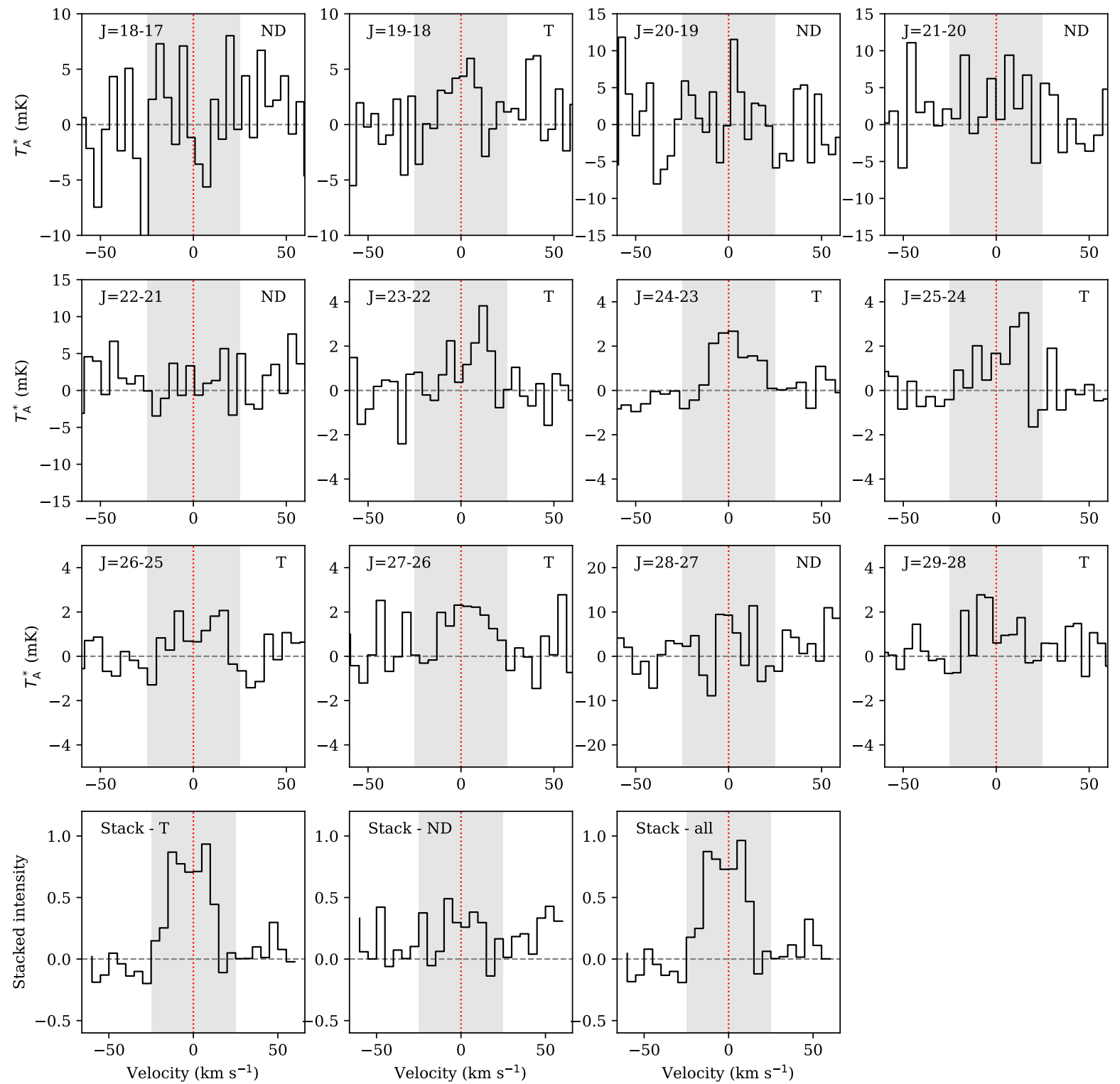

Fig. 6. Detection of $\mathrm{HC}_{3} \mathrm{~N}$ emission. Top three rows: spectra extracted around all frequencies of $\mathrm{HC}_{3} \mathrm{~N}$ rotational transitions in the vibrational ground state $v=0$ covered by our observations. Shaded areas indicate a velocity range $[-25,25] \mathrm{km} \mathrm{s}^{-1}$ around the rest frequency of the transition, and vertical red lines correspond to the rest frequency centred to a velocity of $0 \mathrm{~km} \mathrm{~s}^{-1}$. All panels are labelled with the transition and whether the line is considered tentatively detected (T) or not detected (ND). Bottom row: result of stacking the emission for selected transitions, aligned in velocity space: left: tentatively identified transitions (marked T), middle: transitions not detected (marked ND), right: all twelve transitions shown in the top three rows. We note that the intensity scale is normalised to the peak and has no physical meaning as such.

when stacking the tentative detections, do not find significant structure when stacking the non-detections, and find that the tentative detections weigh strong enough to give rise to line-like structure also in the total stacked profile. This is a direct result of the higher rms noise in the parts of the spectrum with the non-detections, making these less significant.

Based on the multiple tentative detections and the results from the stacking procedure we are confident that we detect $\mathrm{HC}_{3} \mathrm{~N}$ in the CSE of W Aql. To the best of our knowledge, it is the first time such a carbon-chain molecule has been seen in the CSE of an S-type star and it has not yet been detected in the CSE of an M-type AGB star. Comparing the line peak intensities to those reported by Tenenbaum et al. (2010) for IRC +10216 , we find that the emission is weaker in W Aql by a factor 10-15, accounting for the difference in distance and mass-loss rate. This implies that $\mathrm{HC}_{3} \mathrm{~N}$ is roughly one to two orders of magnitude less abundant in the CSE of W Aql than around IRC +10 216 and leads to a first estimate of a fractional abundance on the order $10^{-7}-10^{-8}$ (Agúndez 2009).

\subsubsection{Tentative detections}

HNC. We tentatively detect emission from $\mathrm{HN}^{13} \mathrm{C}(J=3-2)$ at $261.3 \mathrm{GHz}$ at a peak-signal-to-noise ratio (peak-S/N) of $\approx 3$; see Fig. 7a. For this tentative detection, and those of $\mathrm{NaCl}$ and $\mathrm{HCO}^{+}$discussed below, we quote $\mathrm{S} / \mathrm{N}$ values of line profile fits based on the "shell" profile as defined in the GILDAS/CLASS manual, using the rms noise in the line-free part of the spectra at $[-200 ;-50] \mathrm{km} \mathrm{s}^{-1}$.

We do not detect emission from $\mathrm{HN}^{13} \mathrm{C}(J=2-1)$ at $174.2 \mathrm{GHz}$ or $\mathrm{HNC}(J=2-1)$ at $181.3 \mathrm{GHz}$, in line with the 


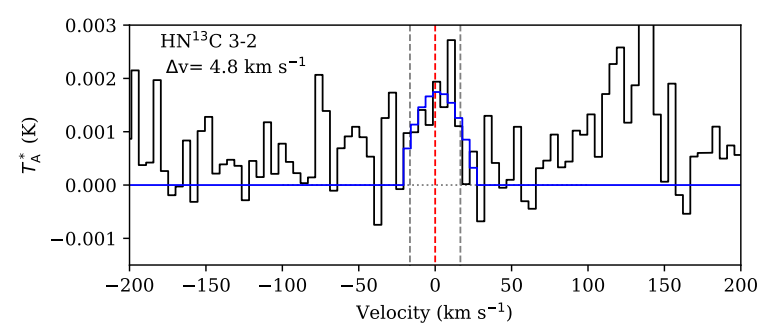

(a) $\mathrm{HN}^{13} \mathrm{C}$
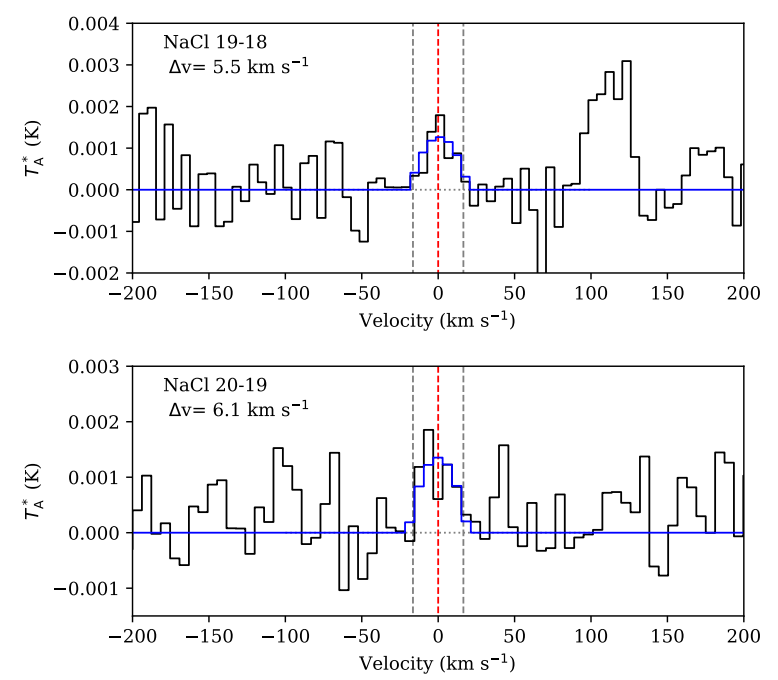

(b) $\mathrm{NaCl}$
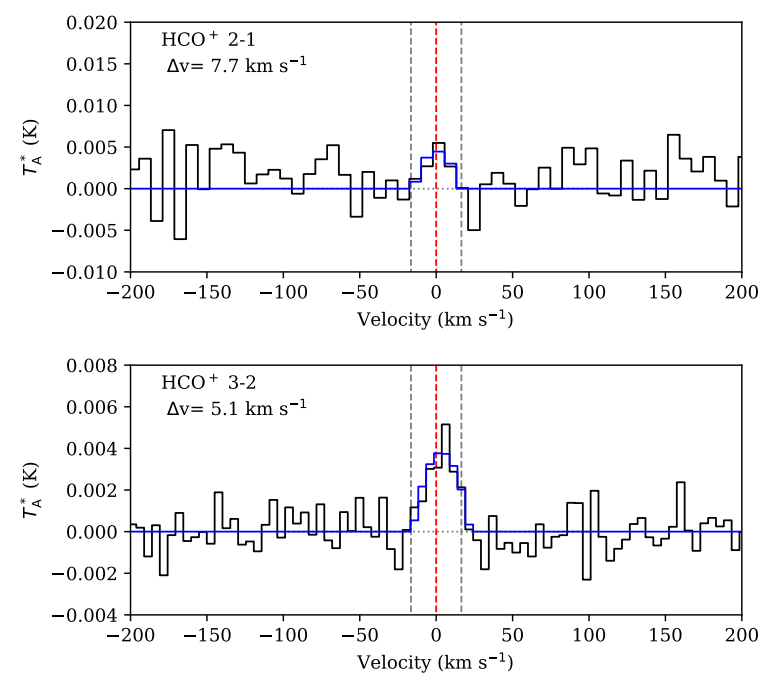

(c) $\mathrm{HCO}^{+}$

Fig. 7. Tentative detections. Red vertical lines correspond to the rest frequencies of the transitions; grey vertical lines indicate the $16.5 \mathrm{~km} \mathrm{~s}^{-1}$ expansion velocity of the CSE of W Aql ; blue fits were produced using the "shell" profile function as defined in the GILDAS/CLASS package. The velocity resolution at which the spectra are presented is listed in each panel.

sensitivity reached in our data set at the relevant frequencies. Assuming optically thin emission from both $\mathrm{H}^{13} \mathrm{CN}$ and $\mathrm{HN}^{13} \mathrm{C}$ we obtain an abundance ratio $\mathrm{HN}^{13} \mathrm{C} / \mathrm{H}^{13} \mathrm{CN}$ of the order $0.01-0.1$. Values reported in the literature for the abundance ratio $\mathrm{HNC} / \mathrm{HCN}$ are anywhere in the range 0.001 and 0.1 for the CSEs of the C-type AGB star IRC +10216 and the supergiant VY CMa (Johansson et al. 1984; Daniel et al. 2012;
Ziurys et al. 2009). For the remainder of the paper, we assume that $\mathrm{HNC} / \mathrm{H}_{2}=0.01 \times \mathrm{HCN} / \mathrm{H}_{2}=3.1 \times 10^{-8}$ (Danilovich et al. 2014) with one order of magnitude uncertainty in both directions, in agreement with our $\mathrm{HN}^{13} \mathrm{C}$ measurements and the above literature values.

$\mathrm{NaCl}$. We tentatively detect $\mathrm{NaCl}(J=19-18,20-19)$ at 247.2 and $260.2 \mathrm{GHz}$; see Fig. 7b. We obtain peak-S/N of $\approx 2$ and integrated-signal-to-noise ratios of $\approx 4$ for both transitions. The non-detections of $\mathrm{NaCl}$ emission in the SEPIAB5 data are consistent with the higher rms noise in those observations. Based on a comparison to the IK Tau spectrum presented by Velilla Prieto et al. (2017) we estimate that $\left(\mathrm{NaCl} / \mathrm{H}_{2}\right)_{\mathrm{W} \mathrm{Aql}} \approx 0.1\left(\mathrm{NaCl} / \mathrm{H}_{2}\right)_{\mathrm{IK} \text { Tau }}$; and based on the $\mathrm{NaCl}$ detections towards IRC +10216 (Agúndez et al. 2012) we estimate that $\left(\mathrm{NaCl} / \mathrm{H}_{2}\right)_{\mathrm{W} \mathrm{Aql}} \approx 4\left(\mathrm{NaCl} / \mathrm{H}_{2}\right)_{\mathrm{IRC}+10216}$. This leads to an estimate for $\left(\mathrm{NaCl} / \mathrm{H}_{2}\right)_{\mathrm{W} \text { Aql }}$ on the order of a few $10^{-8}$. The current data do not provide reliable information on which part of the CSE this emission originates from and follow-up observations at higher sensitivity are needed to draw any firm conclusions on the presence, abundance, and/or location of $\mathrm{NaCl}$ in the CSE. $\mathrm{NaCl}$ emission has been detected around both $\mathrm{M}$ type and C-type AGB stars (e.g. Agúndez et al. 2012; De Beck et al. 2015; De Beck \& Olofsson 2018), but we are not aware of any reported detection towards $\mathrm{S}$-type stars.

$\mathrm{HCO}^{+}$. We tentatively detect emission from $\mathrm{HCO}^{+}$ $(J=3-2)$ at $267.6 \mathrm{GHz}$ at a peak-signal-to-noise ratio of $\approx 4$; see Fig. 7c. There clearly is emission at this position in the spectrum, but we cannot firmly claim that $\mathrm{HCO}^{+}$is the carrier since we have no other detected transition towards W Aql to confirm this. However, based on the tentative identification, we also present the spectrum at $178.4 \mathrm{GHz}$, where the $\mathrm{HCO}^{+}(J=2-1)$ transition lies. We cannot claim a detection, but do observe structure in the spectrum suggestive of a low-intensity emission line at the correct position. Confirmation will require sensitive follow-up observations of multiple transitions.

$\mathrm{HCO}^{+}$has been detected in the CSEs of the M-type AGB stars IK Tau, TX Cam, and W Hya (Pulliam et al. 2011), in the bipolar lobes around the peculiar OH/IR star OH 231.8+4.2 (Sánchez Contreras et al. 2015), and around the supergiants VY CMa and NML Cyg (Ziurys et al. 2007, 2009; Pulliam et al. 2011). All of these CSEs display an oxygen-rich chemistry. Typical abundances $\mathrm{HCO}^{+} / \mathrm{H}_{2}$ range around $10^{-8}-10^{-7}$. $\mathrm{HCO}^{+}$ has also been detected in the CSE of IRC +10216 (Agúndez \& Cernicharo 2006; Pulliam et al. 2011, and references therein), but its abundance in that case is as low as a few $10^{-9}$. IRC +10216 is currently the only C-type AGB star for which such a detection has been reported. The emission (suspected) from $\mathrm{HCO}^{+}(3-2)$ towards W Aql is a factor of roughly 3, 7, and 40 stronger than those reported by Pulliam et al. (2011) towards TX Cam, IK Tau, and IRC +10216 , respectively, accounting for the massloss rates, distances, and difference in beam size between APEX and the SMT. We assume for the remainder of the paper that $\left(\mathrm{HCO}^{+} / \mathrm{H}_{2}\right)_{\mathrm{W} \text { Aql }} \lesssim 7 \times\left(\mathrm{HCO}^{+} / \mathrm{H}_{2}\right)_{\mathrm{IK} \text { Tau }}=7.0 \times 10^{-8}($ Velilla Prieto et al. 2017).

\subsubsection{Upper limits}

$\mathrm{SO}, \mathrm{SO}_{2}$. We do not detect emission from $\mathrm{SO}$ or $\mathrm{SO}_{2}$ in our spectra of W Aql, whereas these are dominant in the spectra of the M-type AGB stars R Dor and IK Tau (De Beck \& Olofsson 2018; Velilla Prieto et al. 2017). From a comparison with scaled 
spectra of R Dor and IK Tau in the SEPIA/B5 range (De Beck \& Olofsson 2018; Velilla Prieto et al. 2017, and De Beck et al., in prep.), accounting for the differences in distance and mass-loss rate $\left(d_{\mathrm{R} \text { Dor }}=59 \mathrm{pc}, \dot{M}_{\mathrm{R} \text { Dor }}=2 \times 10^{-7} M_{\odot} \mathrm{yr}^{-1}, d_{\mathrm{IK} \text { Tau }}=250 \mathrm{pc}\right.$, $\dot{M}_{\text {IK Tau }}=8 \times 10^{-6} M_{\odot} \mathrm{yr}^{-1}$; Maercker et al. 2016; Decin et al. 2010), we set the $\mathrm{SO}_{2}$ abundance of R Dor $\left(5 \times 10^{-6}\right)$ and the SO abundance of IK Tau $\left(1 \times 10^{-6}\right)$ as upper limits for the respective abundances in the CSE of W Aql. We discuss these differences and possible implications for the sulphur chemistry in Sect. 4.

$\mathrm{H}_{2} \mathrm{~S}$. We do not detect emission from $\mathrm{H}_{2} \mathrm{~S}$ in our spectra, although we obtained data at slightly higher sensitivity than Danilovich et al. (2017). From a direct comparison to the line emission from IK Tau reported by Danilovich et al. (2017), we set an upper limit $\left(\mathrm{H}_{2} \mathrm{~S} / \mathrm{H}_{2}\right)_{\mathrm{W} \mathrm{Aql}}<\frac{1}{3} \times\left(\mathrm{H}_{2} \mathrm{~S} / \mathrm{H}_{2}\right)_{\mathrm{IK} \text { Tau }}=5 \times 10^{-7}$ (Danilovich et al. 2017).

$P N, P O$. Similarly, we derive an upper limit $\left(\mathrm{PN} / \mathrm{H}_{2}\right)_{\mathrm{W} \text { Aql }}<$ $\left(\mathrm{PN} / \mathrm{H}_{2}\right)_{\text {IK Tau }}=3 \times 10^{-7}$ based on the comparison of our observations to the emission spectrum of $\mathrm{PN}(J=5-4)$ presented by Velilla Prieto et al. (2017). A comparison to the spectra presented by Milam et al. (2008) for IRC +10 216 does not set additional constraints as the scaled intensity lies far below the sensitivity reached in our observations. Unfortunately, we cannot derive an upper limit for the PO abundance towards W Aql as the sensitivity of the IK Tau spectrum obtained with SEPIA/B5 (scaled for $d$ and $\dot{M}$ ) is superior by a factor of approximately eight to that of the W Aql spectrum and the PO transition at $196 \mathrm{GHz}$ remains undetected in both spectra.

\subsubsection{Unidentified lines}

We find only two unidentified features in this rather broad spectral coverage. Figure B.7 shows emission features in our spectra that we cannot identify with any known carriers. We can rule out contamination of the spectrum through the image sideband for all of these. Two of the three features lie immediately adjacent to each other, are similar in intensity, and are located close to the ${ }^{29} \mathrm{SiS}(v=3, J=14-13)$ line. With the current sensitivity of the data we cannot rule out that these two features (at $249393 \pm 4$ and $\approx 249410 \mathrm{MHz}$ ) are actually part of the same emission line, centred at $249402 \pm 4 \mathrm{MHz}$.

Another unidentified feature is centred at $267073 \pm 4 \mathrm{MHz}$ and seems double-peaked. We cannot identify any possible carrier within $15 \mathrm{MHz}$ of the presumed central frequency.

\subsection{Isotopes}

Isotopic ratios can ideally be used to help constrain the initial mass of the star and its current evolutionary status. We present estimates of isotopic abundance ratios in Table 3 . These are assumed to be represented by the abundance ratios $R_{\mathrm{a} / \mathrm{b}}$ of isotopologues $a$ and $b$, estimated according to

$R_{\mathrm{a} / \mathrm{b}}=\frac{I_{\mathrm{a}\left(J-J^{\prime}\right)}}{I_{\mathrm{b}\left(J-J^{\prime}\right)}} \times\left(\frac{v_{\mathrm{a}\left(J-J^{\prime}\right)}}{v_{\mathrm{b}\left(J-J^{\prime}\right)}}\right)^{-3}$,

using the integrated line strengths $I$ of transitions $J-J^{\prime}$ for isotopologues $\mathrm{a}$ and $\mathrm{b}$ and taking into account the difference in intrinsic line strengths between the isotopologue transitions and telescope beamwidths at the rest frequencies. This simple method assumes that the same transition $J-J^{\prime}$ for two isotopologues is excited under similar conditions.
Table 3. Isotopologue ratios retrieved from frequency-corrected lineintensity ratios $R_{\mathrm{c}}$, as described in the text.

\begin{tabular}{ccrcc}
\hline \hline $\begin{array}{c}\text { Isotopologue } \\
\text { ratio }\end{array}$ & Transition & $R_{\mathrm{c}}$ & \multicolumn{2}{c}{$\begin{array}{c}\text { Solar } \\
\text { isotope ratio }\end{array}$} \\
\hline${ }^{12} \mathrm{CO} /{ }^{13} \mathrm{CO}$ & $2-1$ & $12.3 \pm 0.5$ & ${ }^{12} \mathrm{C} /{ }^{13} \mathrm{C}$ & 89 \\
$\mathrm{H}^{12} \mathrm{CN} / \mathrm{H}^{13} \mathrm{CN}$ & $2-1$ & $5.1 \pm 0.9$ & & \\
${ }^{12} \mathrm{CS} /{ }^{13} \mathrm{CS}$ & $5-4$ & $21.7 \pm 3.4$ & & \\
${ }^{12} \mathrm{CN} /{ }^{13} \mathrm{CN}$ & $2-1$ & $16.7 \pm 4.2$ & & \\
\hline${ }^{28} \mathrm{SiO} /{ }^{29} \mathrm{SiO}$ & $4-3$ & $7.5 \pm 1.3$ & ${ }^{28} \mathrm{Si} /{ }^{29} \mathrm{Si}$ & 20 \\
& $5-4$ & $7.4 \pm 0.6$ & & \\
${ }^{28} \mathrm{SiS} /{ }^{29} \mathrm{SiS}$ & $12-11$ & $20.4 \pm 6.8$ & & \\
& $14-13$ & $12.8 \pm 3.1$ & & \\
${ }^{28} \mathrm{SiO} /{ }^{30} \mathrm{SiO}$ & $4-3$ & $8.4 \pm 1.1$ & ${ }^{28} \mathrm{Si} /{ }^{30} \mathrm{Si}$ & 30 \\
& $6-5$ & $9.3 \pm 1.3$ & & \\
${ }^{28} \mathrm{SiS} /{ }^{30} \mathrm{SiS}$ & $12-11$ & $14.5 \pm 1.9$ & & \\
& $13-12$ & $12.3 \pm 3.1$ & & \\
${ }^{29} \mathrm{SiO} /{ }^{30} \mathrm{SiO}$ & $14-13$ & $28.2 \pm 8.0$ & & \\
${ }^{29} \mathrm{SiS} /{ }^{30} \mathrm{SiS}$ & $12-3$ & $1.1 \pm 0.2$ & ${ }^{29} \mathrm{Si} /{ }^{30} \mathrm{Si}$ & 1.5 \\
& $14-13$ & $0.7 \pm 0.2$ & & \\
& $15-14$ & $2.2 \pm 0.5$ & & \\
\hline${ }^{\mathrm{Si}}{ }^{32} \mathrm{~S} / \mathrm{Si}{ }^{34} \mathrm{~S}$ & $14-13$ & $10.3 \pm 0.1$ & & \\
\hline $\mathrm{C}^{17} \mathrm{O} / \mathrm{C}^{18} \mathrm{O}$ & $2-1$ & $1.0 \pm 0.4$ & ${ }^{17} \mathrm{O} /{ }^{18} \mathrm{O}$ & 0.19 \\
$\mathrm{C}^{16} \mathrm{O} / \mathrm{C}^{18} \mathrm{O}$ & $2-1$ & $355.7 \pm 112.0$ & ${ }^{16} \mathrm{O} /{ }^{18} \mathrm{O}$ & 499 \\
$\mathrm{Si}^{16} \mathrm{O} / \mathrm{Si}{ }^{17} \mathrm{O}$ & $6-5$ & $74.3 \pm 5.4$ & ${ }^{16} \mathrm{O} /{ }^{17} \mathrm{O}$ & 2632 \\
\hline & & & & \\
\hline
\end{tabular}

Notes. We note that the reported errors are formal errors derived from the errors on the respective integrated line strengths. We disregard ${ }^{30} \mathrm{SiO}(J=5-4)$ at $211.853 \mathrm{GHz}$ and $\mathrm{Si}^{34} \mathrm{~S}(J=12-11)$ at $211.854 \mathrm{GHz}$ in this table as they are fully blended. Solar values are taken from Asplund et al. (2009).

Carbon. From radiative transfer modelling of multiple emission lines from both ${ }^{12} \mathrm{CO}$ and ${ }^{13} \mathrm{CO}$, Ramstedt \& Olofsson (2014) and Danilovich et al. (2014) derived ${ }^{12} \mathrm{C} /{ }^{13} \mathrm{C}$ of 26 and 29 , respectively. We do not attempt such modelling here, but it is clear that the $\mathrm{CO}, \mathrm{HCN}, \mathrm{CS}$, and $\mathrm{CN}$ emission are affected by optical-depth effects since the relevant line intensity ratios are much lower than this (see Table 3 ).

Oxygen. The detections of $\mathrm{C}^{17} \mathrm{O}(J=2-1)$ and $\mathrm{C}^{18} \mathrm{O}$ $(J=2-1)$ lead to an estimate of $\mathrm{C}^{17} \mathrm{O} / \mathrm{C}^{18} \mathrm{O}=1.0 \pm 0.4$. We note that the $\mathrm{C}^{18} \mathrm{O}$ emission in our data appears blended with an unidentified source of emission, increasing the uncertainty on this estimate. This component is not visible in the spectra presented by De Nutte et al. (2017) owing to their higher noise. We detect emission from $\operatorname{Si}^{17} \mathrm{O}(J=6-5)$, but not from $\mathrm{Si}^{18} \mathrm{O}(J=6-5)$. This non-detection is in line with the achieved sensitivity, when considering the above results.

Silicon. We detect emission from multiple transitions of ${ }^{28} \mathrm{SiO},{ }^{29} \mathrm{SiO},{ }^{30} \mathrm{SiO}$ and ${ }^{28} \mathrm{SiS},{ }^{29} \mathrm{SiS},{ }^{30} \mathrm{SiS}$; see Figs. B. 3 and B.4. However, based on the ratios sampled by the different transitions, it is clear that optical-depth effects need to be accounted for in order to derive reliable isotopic ratios. For example, we find ${ }^{28} \mathrm{Si} /{ }^{29} \mathrm{Si}$ isotopologue line intensity ratios spanning the range $7-20$ and ${ }^{28} \mathrm{Si} /{ }^{30} \mathrm{Si}$ spanning the range $8-28$. However, detailed abundance modelling using non-local thermodynamical equilibrium (non-LTE) radiative-transfer models is beyond the scope of this paper. 
Sulphur. We detect $\mathrm{Si}^{32} \mathrm{~S}$ and $\mathrm{Si}^{34} \mathrm{~S}$, and $\mathrm{C}^{32} \mathrm{~S}$ and $\mathrm{C}^{34} \mathrm{~S}$. We do not detect any carriers of ${ }^{33} \mathrm{~S}$, the least abundant sulphur isotope. The uneven sampling of the spectrum of the different isotopologues prevents estimates of the isotopic ratios without detailed modelling.

Initial stellar mass. Danilovich et al. (2015) constrained the current mass of $\mathrm{W}$ Aql to be in the range 1.04-3 $M_{\odot}$. De Nutte et al. (2017) derived an initial mass $M_{\mathrm{i}}=1.59 \pm 0.08 M_{\odot}$ based on their analysis of $\mathrm{C}^{17} \mathrm{O}$ and $\mathrm{C}^{18} \mathrm{O}$ emission. Using our observations we derive an initial-mass estimate of $M_{\mathrm{i}}=1.6 \pm 0.2 M_{\odot}$. Both our estimate and the one of De Nutte et al. (2017) are based only on the relative emission strengths of the $J=2-1$ transitions of $\mathrm{C}^{17} \mathrm{O}$ and $\mathrm{C}^{18} \mathrm{O}$. The derived initial mass appears consistent with the fact that evolutionary models predict only stars with initial mass above $\sim 1.5 M_{\odot}$ (e.g. Hinkle et al. 2016) to undergo third dredge-up events, a necessary process to bring $s$-process elements to the surface and move the star from M-type to S-type. Hinkle et al. (2016) report that their model for a star with $M_{\mathrm{i}}=1.6 M_{\odot}$ reaches a $\mathrm{C} / \mathrm{O}=1$ when ${ }^{12} \mathrm{C} /{ }^{13} \mathrm{C}$ reaches 28 . The reported $\mathrm{C} / \mathrm{O}$ and ${ }^{12} \mathrm{C} /{ }^{13} \mathrm{C}$ and our derived initial mass therefore all fit into one consistent picture of $\mathrm{W} \mathrm{Aql} \mathrm{as} \mathrm{a} \mathrm{star} \mathrm{close} \mathrm{to}$ its transition from being oxygen-rich to becoming carbon-rich.

\section{Discussion}

Based on the classification scheme of Keenan \& Boeshaar (1980), Danilovich et al. (2015) reported a spectral type S6/6e and a $\mathrm{C} / \mathrm{O}=0.98$, making $\mathrm{W}$ Aql an S-type star that is close to having a carbon-rich atmosphere. On the other hand, based on the presence of emission from $\mathrm{SiN}, \mathrm{SiC}_{2}, \mathrm{C}_{2} \mathrm{H}$, and $\mathrm{HC}_{3} \mathrm{~N}$ in the CSE, one may classify the content of W Aql's CSE as (rather) carbon-rich. This apparent contrast requires further discussion. In this section we compare the appearance of the atmosphere of W Aql and the surrounding circumstellar gas and dust to what we know about other AGB stars.

\subsection{Atmosphere}

Hony et al. (2009) reported the presence of $\mathrm{HCN}, \mathrm{C}_{2} \mathrm{H}_{2}$, and $\mathrm{C}_{3}$ absorption bands towards $\mathrm{W}$ Aql in the mid-infrared ISO data, but did not discuss this. These molecules are typical for Ctype atmospheres, but their formation is maybe possible also in atmospheres of stars with $\mathrm{C} / \mathrm{O} \lesssim 1$ as a consequence of the nonequilibrium chemistry induced by shocks that travel through the atmosphere and can free atomic carbon due to the destruction of, mainly, CO. Although the models presented by Cherchneff (2006) indeed show an increase in $\mathrm{C}_{2} \mathrm{H}_{2}$ abundance in the presence of shocks compared to thermal equilibrium, they also show an approximately three orders of magnitude drop in the $\mathrm{C}_{2} \mathrm{H}_{2}$ abundance at $5 R_{\star}$ when varying $\mathrm{C} / \mathrm{O}$ from 1 down to 0.98 (see their Fig. 7). The predicted fractional abundance in the latter case, $\approx 10^{-9}$, is still more than five orders of magnitude higher than for stars with $\mathrm{C} / \mathrm{O}<0.9$. For reference, IRC +10216 has a derived $\mathrm{C}_{2} \mathrm{H}_{2}$ abundance of $8 \times 10^{-5}$ in the inner CSE (within 40 stellar radii; Fonfría et al. 2008, and references therein). The models presented by Agúndez et al. (2020) indicate that, even under the assumption of chemical equilibrium, the molecular gas around S-type stars with $\mathrm{C} / \mathrm{O} \approx 1$ could resemble that around C-type stars quite closely, with similar abundances of for example $\mathrm{C}_{2} \mathrm{H}_{2}$ and $\mathrm{HCN}$ in the atmosphere for $\mathrm{C} / \mathrm{O}$ in the range 0.98-1.02. The presence of $\mathrm{C}_{2} \mathrm{H}_{2}$ in the stellar atmosphere of W Aql explains the presence of $\mathrm{C}_{2} \mathrm{H}$ in its circumstellar environment, as the radical is the direct photodissociation product. The presence of both $\mathrm{C}_{2} \mathrm{H}_{2}$ and $\mathrm{HCN}$ in the atmosphere can explain the presence of $\mathrm{HC}_{3} \mathrm{~N}$ in the CSE according to the chemical models of Cordiner \& Millar (2009).

W Aql is a Mira star with a variability in the visual $\Delta V \approx$ 7 mag. Such stars populate region $C$ in the $(K-[12]$, [25] - [60]) colour-colour diagrams presented by Jorissen \& Knapp (1998). Van Eck et al. (2017) point out that strong variability can significantly affect the estimated atmospheric parameters such as the effective temperature, surface gravity, $\mathrm{C} / \mathrm{O}$, and s-process enrichment $[\mathrm{s} / \mathrm{Fe}]$. Keenan \& Boeshaar (1980) report observations of W Aql at only three different phases, making it difficult to assess this potential variability. It is beyond the scope of this paper to quantify the possible effect of photometric variability on the estimated $\mathrm{C} / \mathrm{O}$. However, we repeat that the absence of $\mathrm{TiO}$ lines and the presence of strong $\mathrm{Na} \mathrm{D}$ lines put $\mathrm{W}$ Aql close to the $\mathrm{C}$-type classification, whereas the presence of $\mathrm{ZrO}$ bands points at W Aql not yet being sufficiently enriched to be C-type.

\subsection{Circumstellar gas}

Figure 8 is central to our discussion on how the circumstellar chemistry of W Aql compares to that of other AGB CSEs. We show estimated peak fractional abundances for CSEs around a small collection of AGB stars: the two M-type stars R Dor and IK Tau, the two S-type stars $\chi$ Cyg and W Aql, and the two C-type stars V Aql and IRC +10216 . For all three chemical types the former star has a mass-loss rate in the range $0.1-0.7 \times$ $10^{-6} M_{\odot} \mathrm{yr}^{-1}$ and the latter in the range 3.0-20 $\times 10^{-6} M_{\odot} \mathrm{yr}^{-1}$. For a few molecules, we also show median values for different chemical types based on sample studies ${ }^{8}$ and list the used peak fractional abundances and the references to the relevant studies in Table C.1.

Danilovich et al. (2014) found that their derived circumstellar abundances of $\mathrm{HCN}, \mathrm{SiO}$, and $\mathrm{H}_{2} \mathrm{O}$ for $\mathrm{W}$ Aql are between those typical for M- and C-type AGB stars, from which they conclude that the abundances are consistent with an S-type classification. Schöier et al. (2013) reported that the HCN abundance in S-type CSEs is indeed intermediate between those in CSEs of M-type and C-type AGB stars.

Contrary to the fact that the CSE of W Aql seems to have chemical properties intermediate between the M-type and C-type classes when looking at the most common candidates such as $\mathrm{CS}, \mathrm{SiS}, \mathrm{HCN}$, and $\mathrm{SiO}$, we estimate high abundances of $\mathrm{C}_{2} \mathrm{H}, \mathrm{CN}, \mathrm{SiC}_{2}$, and $\mathrm{SiN}$, and likely also of $\mathrm{HC}_{3} \mathrm{~N}$, and HNC. All of these molecules are expected around C-type stars and are not seen around M-type stars. Given that $\mathrm{C} / \mathrm{O}<1$ for S-type stars like W Aql, one does not, at first sight, expect such a carbon-rich chemistry as we report in Sect. 3 and show in Fig. 8. For molecules where CSEs from M-type stars exhibit markedly higher abundances than those from C-type stars, for example $\mathrm{H}_{2} \mathrm{~S}, \mathrm{PN}, \mathrm{PO}, \mathrm{SO}$, and $\mathrm{SO}_{2}$, we have no estimates or only upper limits for the case of $\mathrm{W}$ Aql. From our results and the abundances reported in the literature and compiled in Fig. 8 it seems that W Aql's CSE is closer to that of IRC +10216 in its gas-phase chemistry than to that of IK Tau, whereas all three stars have roughly similar wind properties (expansion velocity and mass-loss rate). Only in the cases of $\mathrm{H}_{2} \mathrm{O}$ and $\mathrm{NH}_{3}$ are the abundances for W Aql closer to those for IK Tau than to those for IRC +10216 ; for $\mathrm{SiO}$, the reported abundance is exactly

\footnotetext{
8 We wish to remark, however, that some sample sizes are limited and reported values are in those cases likely biased towards high abundances. We refer to the papers in question for details on samples and biases.
} 


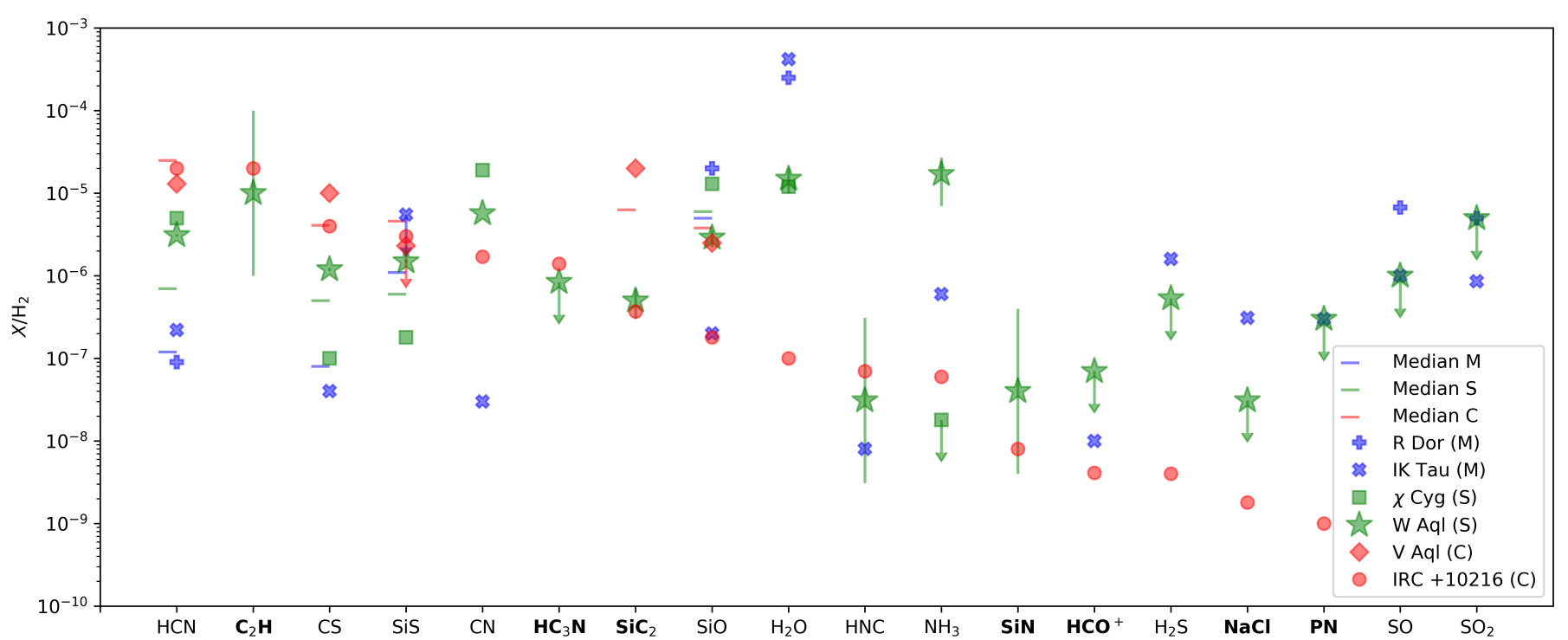

Fig. 8. Peak molecular abundances in the CSEs of AGB stars of different chemical types: M-type stars R Dor and IK Tau, S-type stars $\chi$ Cyg and W Aql, and C-type stars V Aql and IRC +10 216. We note that the high SiS abundance for IK Tau is only representative of a geometrically small component centred on the star, after which depletion is very efficient and the abundance drops roughly four orders of magnitude. See text for further discussion and references. We have included error bars only for W Aql for the sake of visibility. The error bars correspond to the values specified in Table C.1 and we note that they are often smaller than the plotting symbol. Upper limits are indicated with downwards arrows. Molecules that were not previously identified towards W Aql are highlighted in boldface font on the horizontal axis.

intermediate ${ }^{9}$. In the case of $\mathrm{NH}_{3}, \mathrm{~W}$ Aql's abundance exceeds that of IK Tau, removing it entirely from an intermediate status. We note, though, that the excitation of all three molecules $\left(\mathrm{H}_{2} \mathrm{O}\right.$, $\mathrm{NH}_{3}$, and $\mathrm{SiO}$ ) is predominantly through the strongly variable radiation field, possibly introducing considerable uncertainties on the derived abundances (for all objects).

Currently, it is difficult to assess the differences or similarities between the CSE of $\mathrm{W} A q 1(\mathrm{C} / \mathrm{O}=0.98)$ on the one hand, and that of $\chi \mathrm{Cyg}(\mathrm{C} / \mathrm{O} \leq 0.95$; based on the measurements and classification scheme presented by Keenan \& Boeshaar 1980) on the other, as broad, sensitive observations of $\chi$ Cyg are currently missing. The chemical equilibrium models of Agúndez et al. (2020) provide support for an inner-wind chemistry around W Aql closely resembling that of a C-type AGB star. However, the molecules we detect that are "typical for C-rich CSEs" are found in the outer CSE and there are currently no chemical models available that describe the chemical content of the CSEs of S-type AGB stars on that physical scale. Because of this and the lack of observational characterisation of the CSEs of S-type stars with different $\mathrm{C} / \mathrm{O}$, we cannot yet assess in a straightforward way how (un)typical the CSE of W Aql is. Chemical models must explore the impact of the atmospheric $\mathrm{C} / \mathrm{O}$ on the circumstellar abundances.

\subsection{Dust}

Hony et al. (2009) proposed the presence of non-stoichiometric silicates around S-type stars to explain the substantial spectral differences in the infrared between CSEs of S-type and M-type AGB stars, which is plausible since stars with $\mathrm{C} / \mathrm{O} \sim 1$ exhibit an increased $\mathrm{Mg} / \mathrm{SiO}_{4}$ abundance ratio, with less free oxygen available when dust forms. Additionally, the latter authors

9 We cannot make such a claim with certainty in the cases of $\mathrm{HCO}^{+}$and $\mathrm{NaCl}$, since our detections are tentative and our abundance estimates very rough. Differences within an order of magnitude are hence not significant at this point. reported the presence of the $30 \mu \mathrm{m}$ feature, attributed to $\mathrm{MgS}$, in the ISO spectrum of W Aql and $\pi^{1}$ Gru, another S-type star. This feature is typical for carbon-rich CSEs and is seen only for S-type AGB stars with a C/O $>0.96$ (Smolders et al. 2012, their group III stars). $\mathrm{MgS}$ is suggested to be present in mantles covering dust grains such as for example amorphous alumina. No estimate is available for the amount of $\mathrm{S}$ that would be bound in $\mathrm{MgS}$ dust. Hony et al. (2009) also mentioned the possibility of SiC dust in the spectrum of $\mathrm{W} \mathrm{Aql,} \mathrm{at} \sim 11$ and $\sim 17 \mu \mathrm{m}$, another dust species typical of C-rich CSEs. It is unclear whether the W Aql spectrum shows a contribution from amorphous carbon dust, which typically accounts for several tens of percent of the dust mass in carbon-rich CSEs. Furthermore, the W Aql spectrum lacks the amorphous silicate feature at $18 \mu \mathrm{m}$ and lacks a clear silicate feature at $20 \mu \mathrm{m}$. We are not aware of radiative transfer models of W Aql exploring the dust composition in detail.

\subsection{Influence from the binary companion?}

Danilovich et al. (2015) found that the binary companion is a main-sequence dwarf star of spectral type F8 - G0. It is unlikely that this dwarf star has a significant influence on the photochemistry in the CSE of the AGB star. Based on ALMA observations, Ramstedt et al. (2017) show that the binary companion has a rather weak but measurable effect on the CSE morphology. Locally increased densities could cause changes in the circumstellar chemistry. However, the emission lines reported here are detected with APEX, a single-dish telescope, and are most likely more representative of the overall CSE chemistry rather than of localised deviations.

\section{Conclusions}

We observed the CSE of the S-type AGB star W Aql with APEX at 159-211 GHz using the SEPIA/B5 instrument, and in the frequency windows 208.4-239.8, 244.2-255.8, and 
260.0-267.8 GHz using the PI230 instrument. We report a number of new detections towards this CSE, including $\mathrm{C}_{2} \mathrm{H}, \mathrm{SiN}$, $\mathrm{SiC}_{2}$, and $\mathrm{HC}_{3} \mathrm{~N}$, all of which are molecules previously reported only towards C-rich CSEs. We tentatively detect $\mathrm{HN}^{13} \mathrm{C}, \mathrm{NaCl}$, and $\mathrm{HCO}^{+}$. We made some first estimates of the molecular abundances, and compared these to abundances reported for CSEs of M-type and C-type AGB stars. We find that the CSE of W Aql is more similar to that of a C-type AGB star than to that of an M-type AGB star with similar wind properties, in agreement with the recent chemical equilibrium models of Agúndez et al. (2020). We cannot currently assess how typical the abundances in W Aql's CSE are for S-type CSEs: observations have not yet extensively covered the parameter space of S-type star CSEs, in terms of wind density, $\mathrm{C} / \mathrm{O}$, and chemical content, and chemical models of the outer CSE of S-type stars are lacking.

As the ISO observations of W Aql show signatures of $\mathrm{C}_{2} \mathrm{H}_{2}$ and $\mathrm{HCN}$ in the atmosphere and of $\mathrm{MgS}$ and $\mathrm{SiC}$ in the dust, our detections of molecular gas deemed typical for carbon-rich stars should perhaps not be entirely unexpected. However, previous abundance derivations for commonly targeted molecules such as $\mathrm{SiO}, \mathrm{SiS}, \mathrm{HCN}$, and $\mathrm{CS}$ have consistently led to the conclusion that W Aql appears to be a rather typical S-type star, with abundances intermediate to those characteristic for CSEs around C-type and M-type stars. It is only now, with the presented frequency coverage, that the possibly more carbonaceous nature of W Aql's molecular CSE becomes apparent.

We hope that our findings on W Aql can spark increased interest in the chemical diversity in the stellar CSEs that comes along with the chemical evolution of the AGB stars themselves. Broader inventories, both in terms of targets and frequency ranges, of the molecular content of CSEs around M-type and S-type stars in particular are essential to constrain comprehensive chemical models. Strong constraints on the gas content are also essential to understand the dust formation and dust growth taking place close to the star.

Acknowledgements. E.D.B. acknowledges financial support from the Swedish National Space Agency. H.O. acknowledges financial support from the Swedish Research Council. Based on observations with the Atacama Pathfinder EXperiment (APEX) telescope. APEX is a collaboration between the Max Planck Institute for Radio Astronomy, the European Southern Observatory, and the Onsala Space Observatory. Swedish observations on APEX are supported through Swedish Research Council grant No 2017-00648. The APEX observations were obtained under project numbers O-099.F-9306 (SEPIA/B5) and O-0101.F-9312, O-0102.F-9313, and O-0103.F-9319 (PI230).

\section{References}

Agúndez, M. 2009, PhD thesis, Departamento de Astrofisica, Madrid, Spain Agúndez, M., \& Cernicharo, J. 2006, ApJ, 650, 374

Agúndez, M., Fonfría, J. P., Cernicharo, J., et al. 2012, A\&A, 543, A48

Agúndez, M., Martinez, J. I., de Andres, P. L., Cernicharo, J., \& Martin-Gago, J. A. 2020, A\&A 637, A59

Alfonso-Garzón, J., Domingo, A., Mas-Hesse, J. M., \& Giménez, A. 2012, A\&A, 548, A79

Asplund, M., Grevesse, N., Sauval, A. J., \& Scott, P. 2009, ARA\&A, 47, 481

Bachiller, R., Fuente, A., Bujarrabal, V., et al. 1997, A\&A, 319, 235

Belitsky, V., Lapkin, I., Fredrixon, M., et al. 2018, A\&A, 612, A23

Billade, B., Nyström, O., Meledin, D., et al. 2012, IEEE Trans. Terahertz Sci. Technol., 2, 208

Brunner, M., Danilovich, T., Ramstedt, S., et al. 2018, A\&A, 617, A23

Cernicharo, J., Teyssier, D., Quintana-Lacaci, G., et al. 2014, ApJ, 796, L21
Cherchneff, I. 2006, A\&A, 456, 1001

Cherchneff, I. 2012, A\&A, 545, A12

Cordiner, M. A., \& Millar, T. J. 2009, ApJ, 697, 68

Daniel, F., Agúndez, M., Cernicharo, J., et al. 2012, A\&A, 542, A37

Danilovich, T., Bergman, P., Justtanont, K., et al. 2014, A\&A, 569, A76

Danilovich, T., Olofsson, G., Black, J. H., Justtanont, K., \& Olofsson, H. 2015, A\&A, 574, A23

Danilovich, T., De Beck, E., Black, J. H., Olofsson, H., \& Justtanont, K. 2016, A\&A, 588, A119

Danilovich, T., Van de Sande, M., De Beck, E., et al. 2017, A\&A, 606, A124

Danilovich, T., Ramstedt, S., Gobrecht, D., et al. 2018, A\&A, 617, A132

De Beck, E., \& Olofsson, H. 2018, A\&A, 615, A8

De Beck, E., Decin, L., de Koter, A., et al. 2010, A\&A, 523, A18

De Beck, E., Lombaert, R., Agúndez, M., et al. 2012, A\&A, 539, A108

De Beck, E., Kamiński, T., Patel, N. A., et al. 2013, A\&A, 558, A132

De Beck, E., Kamiński, T., Menten, K. M., et al. 2015, ASP Conf. Ser., 497, 73

Decin, L., Cherchneff, I., Hony, S., et al. 2008, A\&A, 480, 431

Decin, L., De Beck, E., Brünken, S., et al. 2010, A\&A, 516, A69

De Nutte, R., Decin, L., Olofsson, H., et al. 2017, A\&A, 600, A71

Fonfría, J. P., Cernicharo, J., Richter, M. J., \& Lacy, J. H. 2008, ApJ, 673, 445

Gobrecht, D., Cherchneff, I., Sarangi, A., Plane, J. M. C., \& Bromley, S. T. 2016, A\&A, 585, A6

González Delgado, D., Olofsson, H., Kerschbaum, F., et al. 2003, A\&A, 411, 123 Güsten, R., Nyman, L. Å., Schilke, P., et al. 2006, A\&A, 454, L13

Habing, H. J., \& Olofsson, H. 2004, Asymptotic Giant Branch Stars (New York: Springer)

Hinkle, K. H., Lebzelter, T., \& Straniero, O. 2016, ApJ, 825, 38

Hony, S., Heras, A. M., Molster, F. J., \& Smolders, K. 2009, A\&A, 501, 609

Humphreys, E. M. L., Immer, K., Gray, M. D., et al. 2017, A\&A, 603, A77

Johansson, L. E. B., Andersson, C., Ellder, J., et al. 1984, A\&A, 130, 227

Jorissen, A., \& Knapp, G. R. 1998, A\&AS, 129, 363

Keenan, P. C., \& Boeshaar, P. C. 1980, ApJS, 43, 379

Maercker, M., Danilovich, T., Olofsson, H., et al. 2016, A\&A, 591, A44

Massalkhi, S., Agúndez, M., Cernicharo, J., et al. 2018, A\&A, 611, A29

Massalkhi, S., Agúndez, M., \& Cernicharo, J. 2019, A\&A, 628, A62

Men'shchikov, A. B., Balega, Y., Blöcker, T., Osterbart, R., \& Weigelt, G. 2001, A\&A, 368, 497

Milam, S. N., Halfen, D. T., Tenenbaum, E. D., et al. 2008, ApJ, 684, 618

Müller, H. S. P., Klaus, T., \& Winnewisser, G. 2000, A\&A, 357, L65

Müller, H. S. P., Thorwirth, S., Roth, D. A., \& Winnewisser, G. 2001, A\&A, 370, L49

Müller, H. S. P., Schlöder, F., Stutzki, J., \& Winnewisser, G. 2005, J. Mol. Struct., 742,215

Pickett, H. M., Poynter, R. L., Cohen, E. A., et al. 1998, J. Quant. Spectr. Rad. Transf., 60, 883

Pulliam, R. L., Edwards, J. L., \& Ziurys, L. M. 2011, ApJ, 743, 36

Ramstedt, S., \& Olofsson, H. 2014, A\&A, 566, A145

Ramstedt, S., Schöier, F. L., \& Olofsson, H. 2009, A\&A, 499, 515

Ramstedt, S., Mohamed, S., Vlemmings, W. H. T, et al. 2017, A\&A, 605, A126

Sánchez Contreras, C., Velilla Prieto, L., Agúndez, M., et al. 2015, A\&A, 577, A52

Schmidt, M. R., He, J. H., Szczerba, R., et al. 2016, A\&A, 592, A131

Schöier, F. L., Olofsson, H., \& Lundgren, A. A. 2006, A\&A, 454, 247

Schöier, F. L., Maercker, M., Justtanont, K., et al. 2011, A\&A, 530, A83

Schöier, F. L., Ramstedt, S., Olofsson, H., et al. 2013, A\&A, 550, A78

Smolders, K., Acke, B., Verhoelst, T., et al. 2010, A\&A, 514, L1

Smolders, K., Neyskens, P., Blommaert, J. A. D. L., et al. 2012, A\&A, 540, A72

Tenenbaum, E. D., Dodd, J. L., Milam, S. N., Woolf, N. J., \& Ziurys, L. M. 2010, ApJS, 190, 348

Turner, B. E. 1992, ApJ, 388, L35

Van de Sande, M., Sundqvist, J. O., Millar, T. J., et al. 2018, A\&A, 616, A106

Van Eck, S., Neyskens, P., Jorissen, A., et al. 2017, A\&A, 601, A10

Velilla Prieto, L., Sánchez Contreras, C., Cernicharo, J., et al. 2017, A\&A, 597. A25

Velilla-Prieto, L., Cernicharo, J., Agúndez, M., et al. 2019, IAU Symp., 343, 535 Willacy, K., \& Millar, T. J. 1997, A\&A, 324, 237

Wong, K. T., Menten, K. M., Kamiński, T., et al. 2018, A\&A, 612, A48

Ziurys, L. M., Milam, S. N., Apponi, A. J., \& Woolf, N. J. 2007, Nature, 447, 1094

Ziurys, L. M., Tenenbaum, E. D., Pulliam, R. L., Woolf, N. J., \& Milam, S. N. 2009, ApJ, 695, 1604 
E. De Beck and H. Olofsson: The surprisingly carbon-rich environment of the S-type star W Aq1

\section{Appendix A: Data presentation}

\section{A.1. Data contamination}

We detect five features in our spectra that are leakage from strong lines in the image sideband of a given frequency setup; see Table A.1. In the SEPIA/B5-data the leakage corresponds to a suppression of the signal at $26 \mathrm{~dB}$, which is significantly better than the average $18.5 \mathrm{~dB}$ suppression reported for SEPIA/B5 (Humphreys et al. 2017; Belitsky et al. 2018). In the PI230 data we find image leakage at suppression levels between 24 and $36 \mathrm{~dB}$.

\section{A.2. Spectra}

We show the complete spectra obtained with SEPIA/B5 in Fig. A.1 and for PI230 in Fig. A.2. Both are displayed at a resolution of $7.5 \mathrm{~km} \mathrm{~s}^{-1}$.

\section{A.3. Line properties}

All features that have been detected in our observations and marked in Figs. A.1 and A.2 are listed in Table A.2. We report
Table A.1. Image sideband leakage from strong lines.

\begin{tabular}{cccc}
\hline \hline $\begin{array}{c}\text { Frequency } \\
(\mathrm{GHz})\end{array}$ & $\begin{array}{c}\text { Molecular } \\
\text { transition }\end{array}$ & $\begin{array}{c}\text { Fraction of signal } \\
\text { intensity }(\%)\end{array}$ & $\begin{array}{c}\text { Suppres- } \\
\text { sion }(\mathrm{dB})\end{array}$ \\
\hline 160.3 & $\mathrm{SiO}(4-3)$ & 5.0 & 26 \\
209.9 & $\mathrm{CO}(2-1)$ & 1.5 & 36 \\
239.3 & $\mathrm{SiO}(5-4)$ & 1.5 & 36 \\
246.1 & $\mathrm{HCN}(3-2)$ & 2.3 & 33 \\
267.1 & $\mathrm{CS}(5-4)$ & 6.2 & 24 \\
\hline
\end{tabular}

the extent of the line in velocity range $\left[v_{\min }, v_{\max }\right]$ and the integrated intensity across this range with a $2 \sigma$ error. Comments in the table relate to, for example, tentative detections, blends, or hyperfine structure. We note that the velocity ranges are not necessarily symmetric around $0 \mathrm{~km} \mathrm{~s}^{-1}$ since they were derived to account for weak wings of emission seen for some of the lines. A detailed study of the physical structures within the CSE traced by different molecular transitions is beyond the scope of this paper and would benefit from spatially resolved observations. 

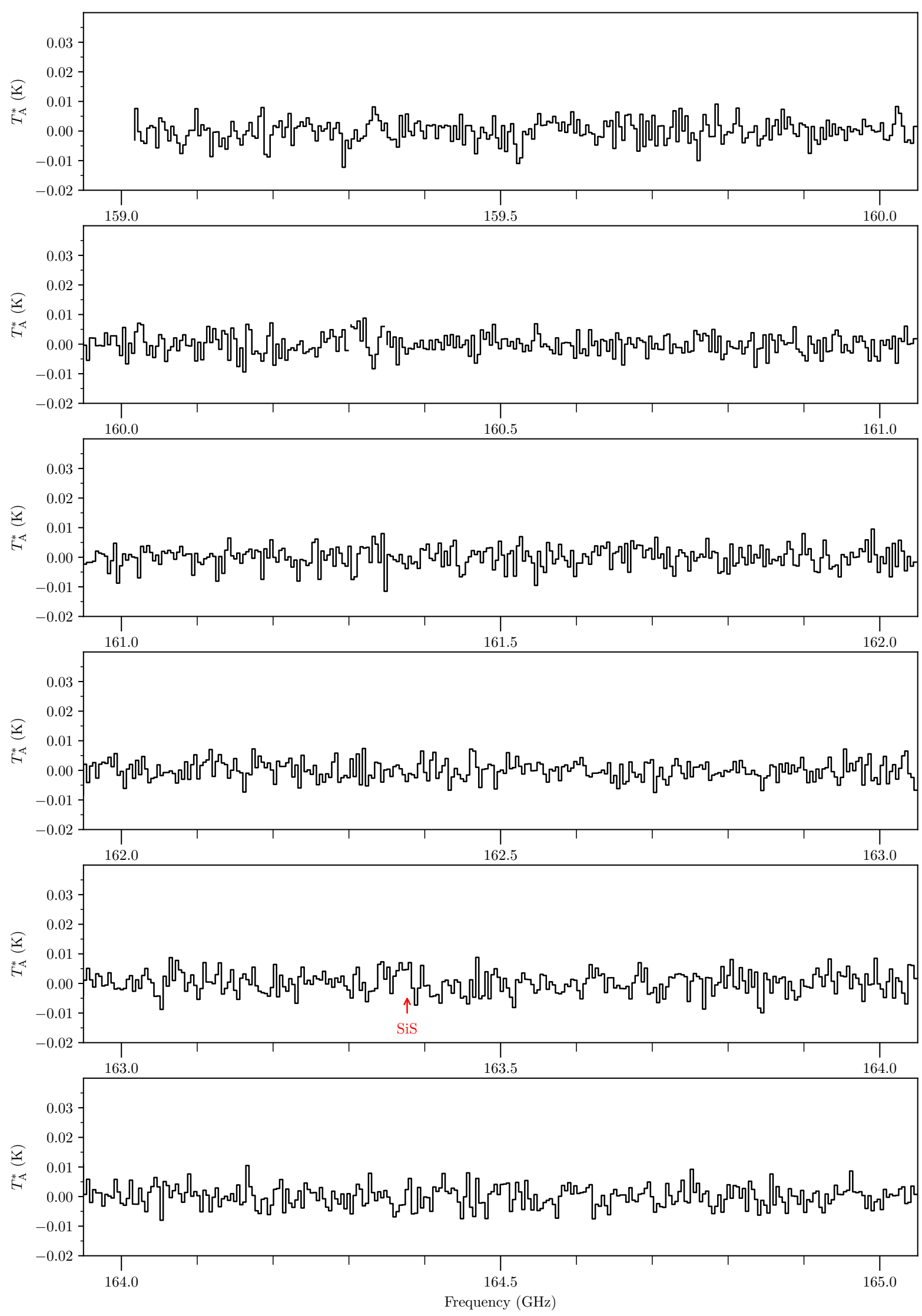

Fig. A.1. APEX SEPIA/B5 line survey of W Aql. Labels show the carrier molecule of the indicated emission. Red labels indicate tentative or unidentified detections. Purple labels $(\operatorname{Im}(\ldots))$ pertain to emission contaminating the signal from the image sideband (see Sect. 2). For visibility, some parts of the survey were rescaled to fit the vertical scale. The colour coding of the spectrum corresponds to the following scale factors: (black) 1; (green) $1 / 5$. We note that the labelling at frequencies above $209.0 \mathrm{GHz}$ is based on identifications in the PI 230 spectrum and is shown here only for reference. 
E. De Beck and H. Olofsson: The surprisingly carbon-rich environment of the S-type star W Aq1
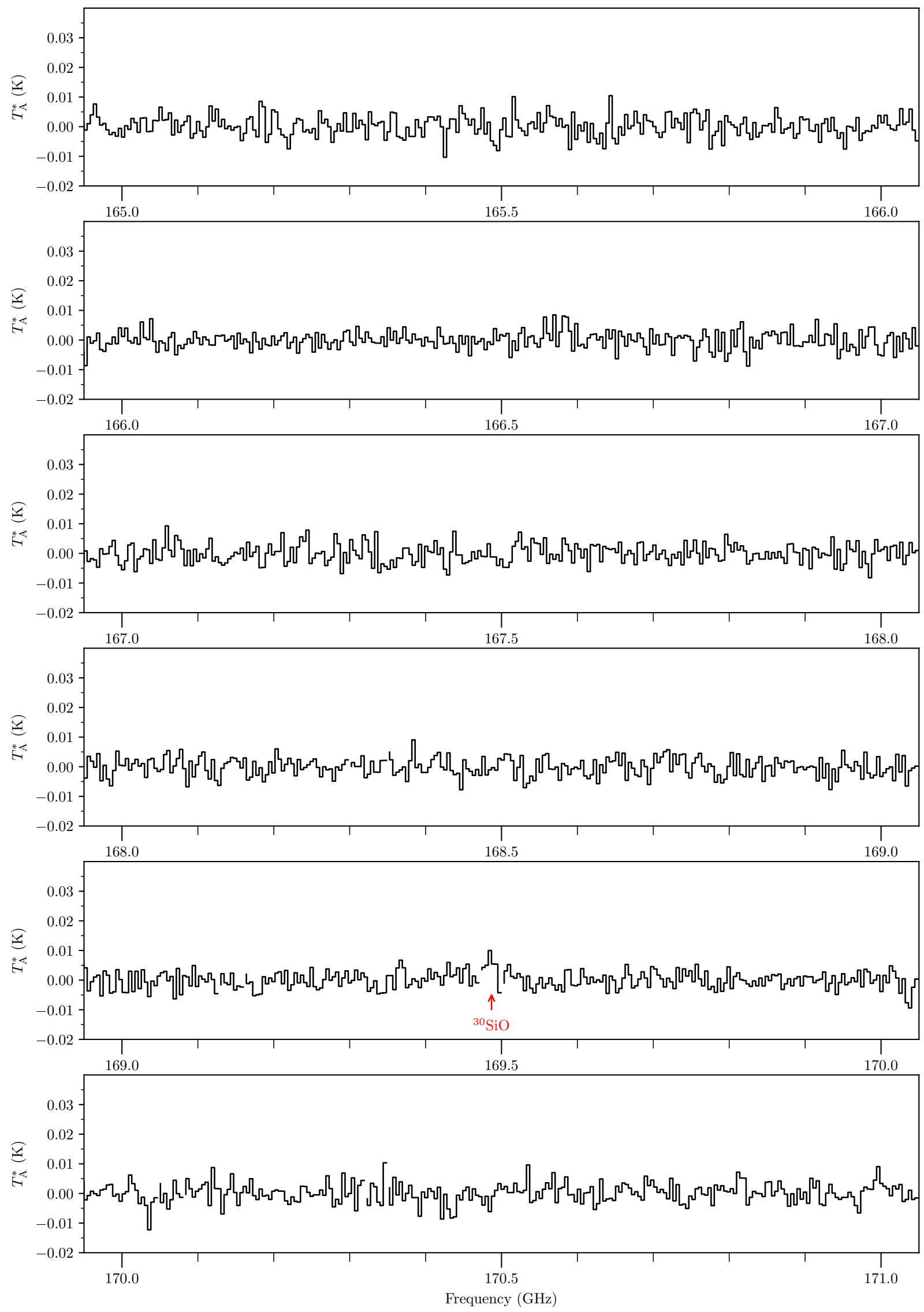

Fig. A.1. continued. 

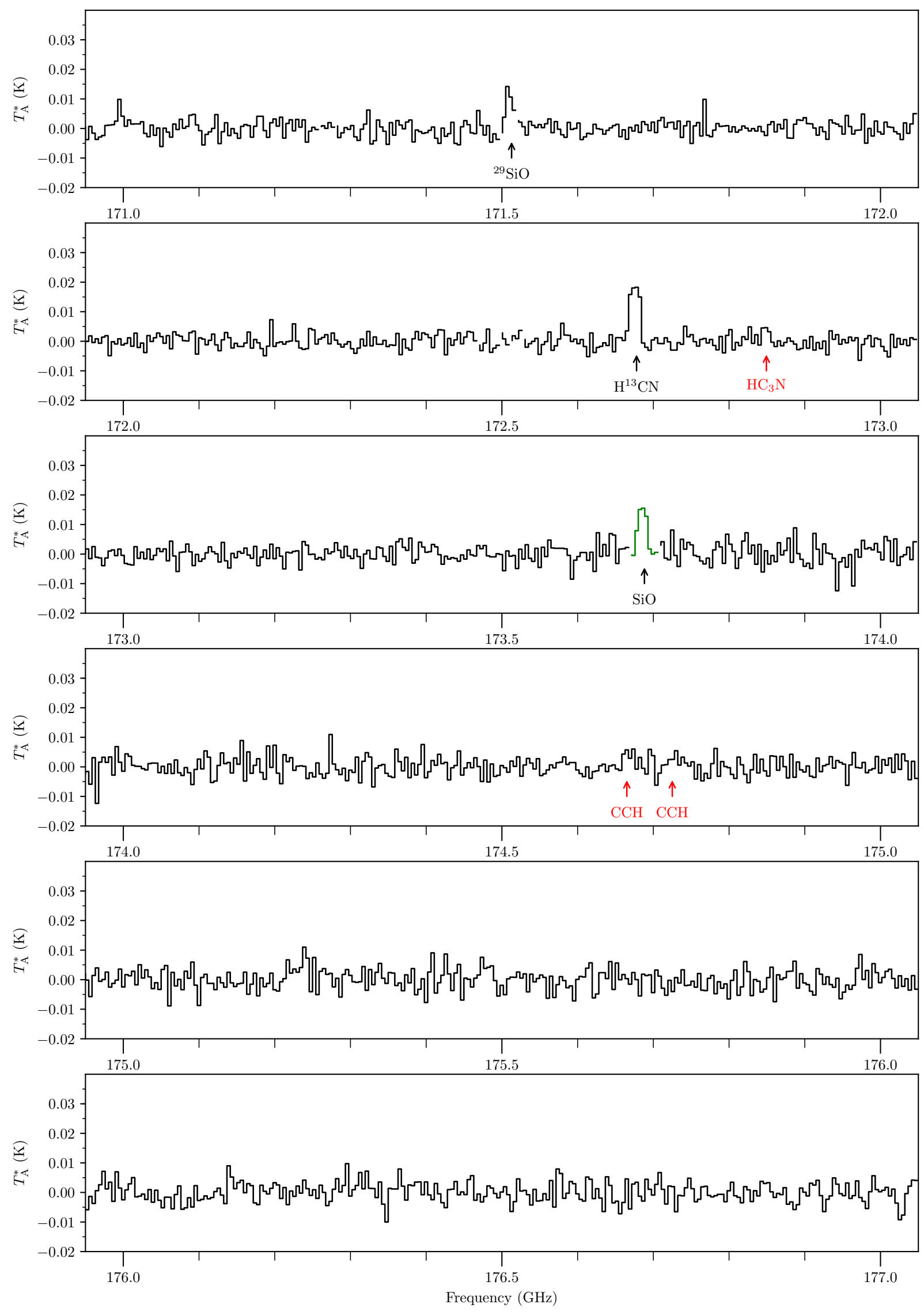

Fig. A.1. continued. 
E. De Beck and H. Olofsson: The surprisingly carbon-rich environment of the S-type star W Aq1
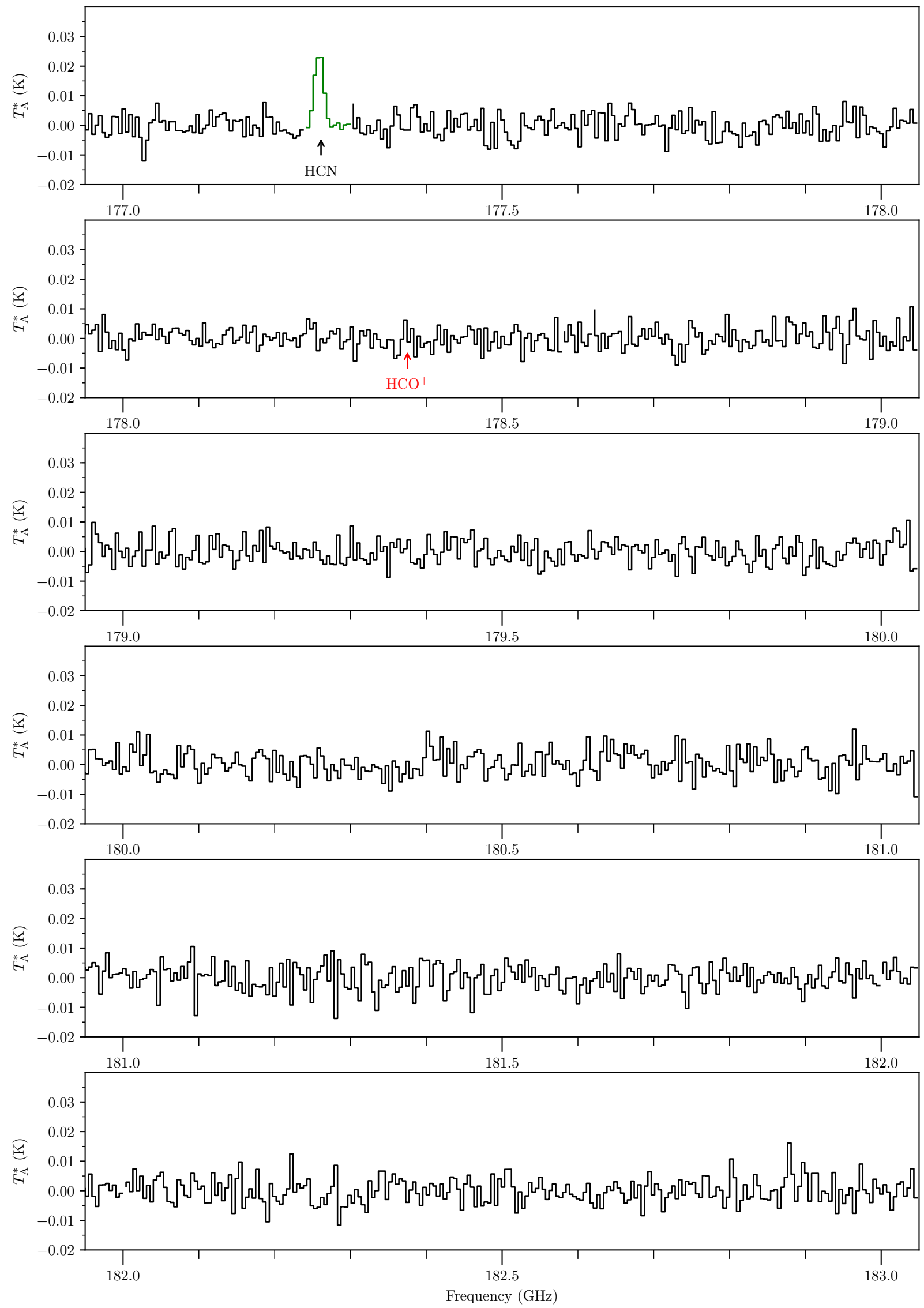

Fig. A.1. continued. 

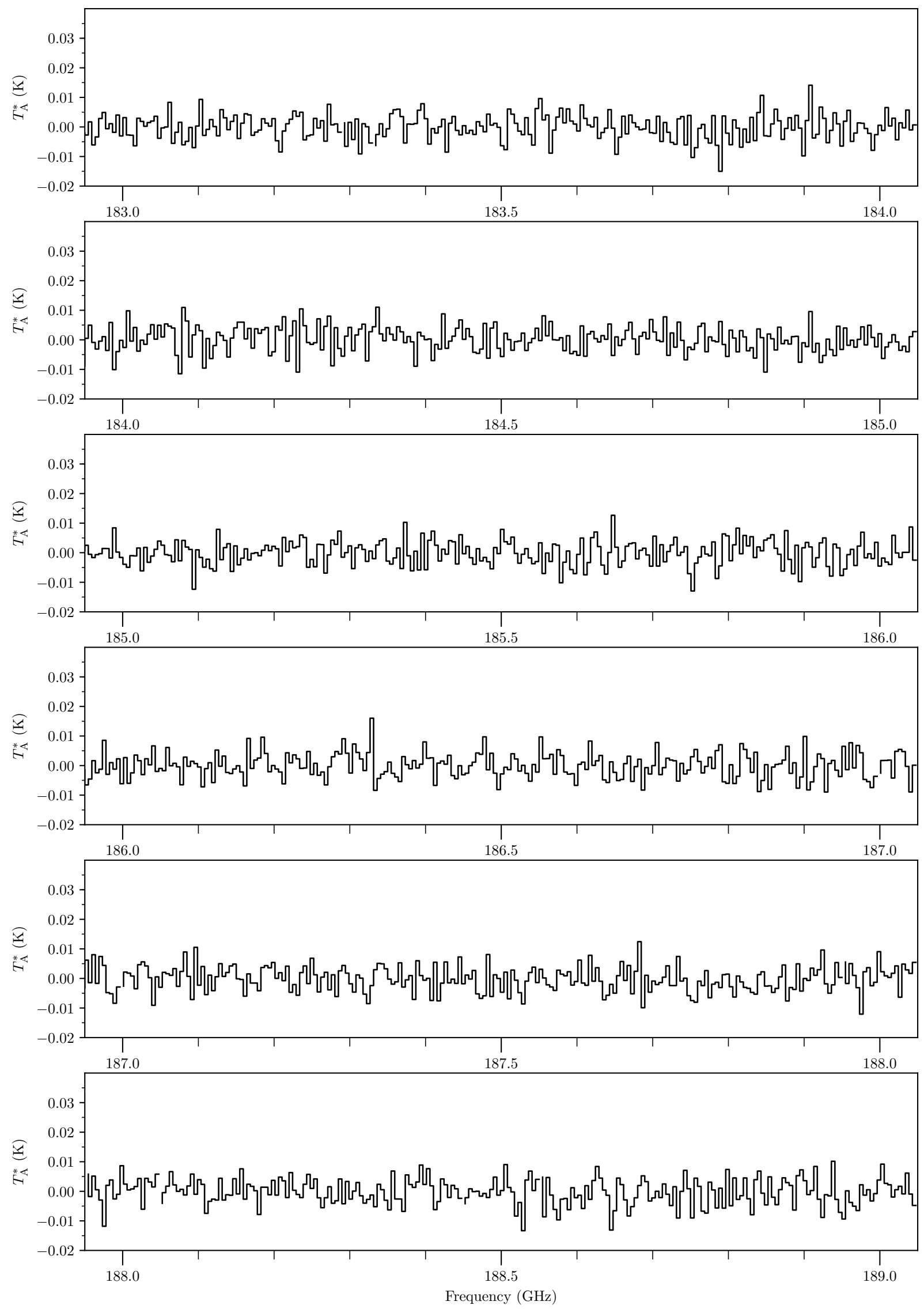

Fig. A.1. continued. 
E. De Beck and H. Olofsson: The surprisingly carbon-rich environment of the S-type star W Aq1
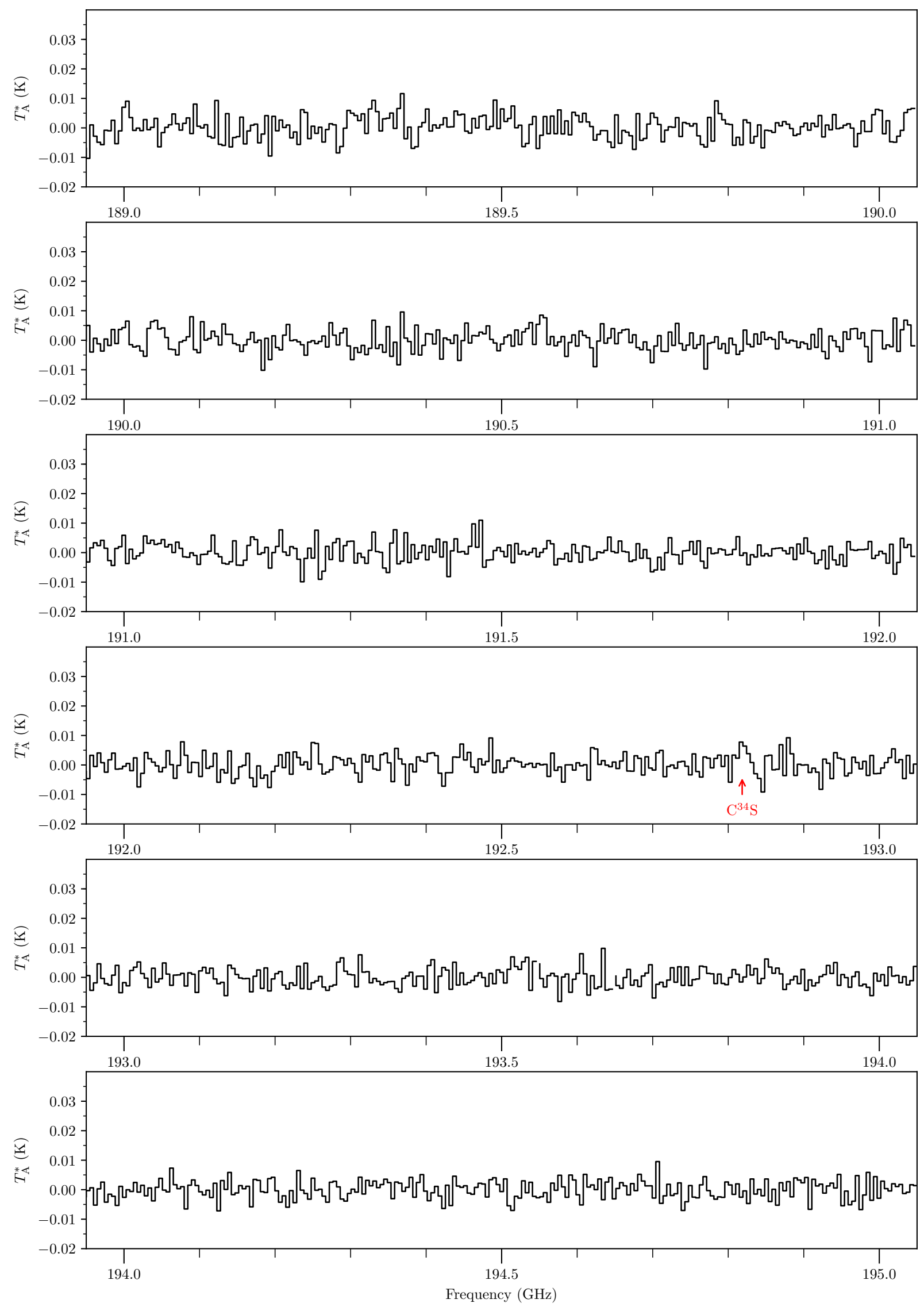

Fig. A.1. continued. 

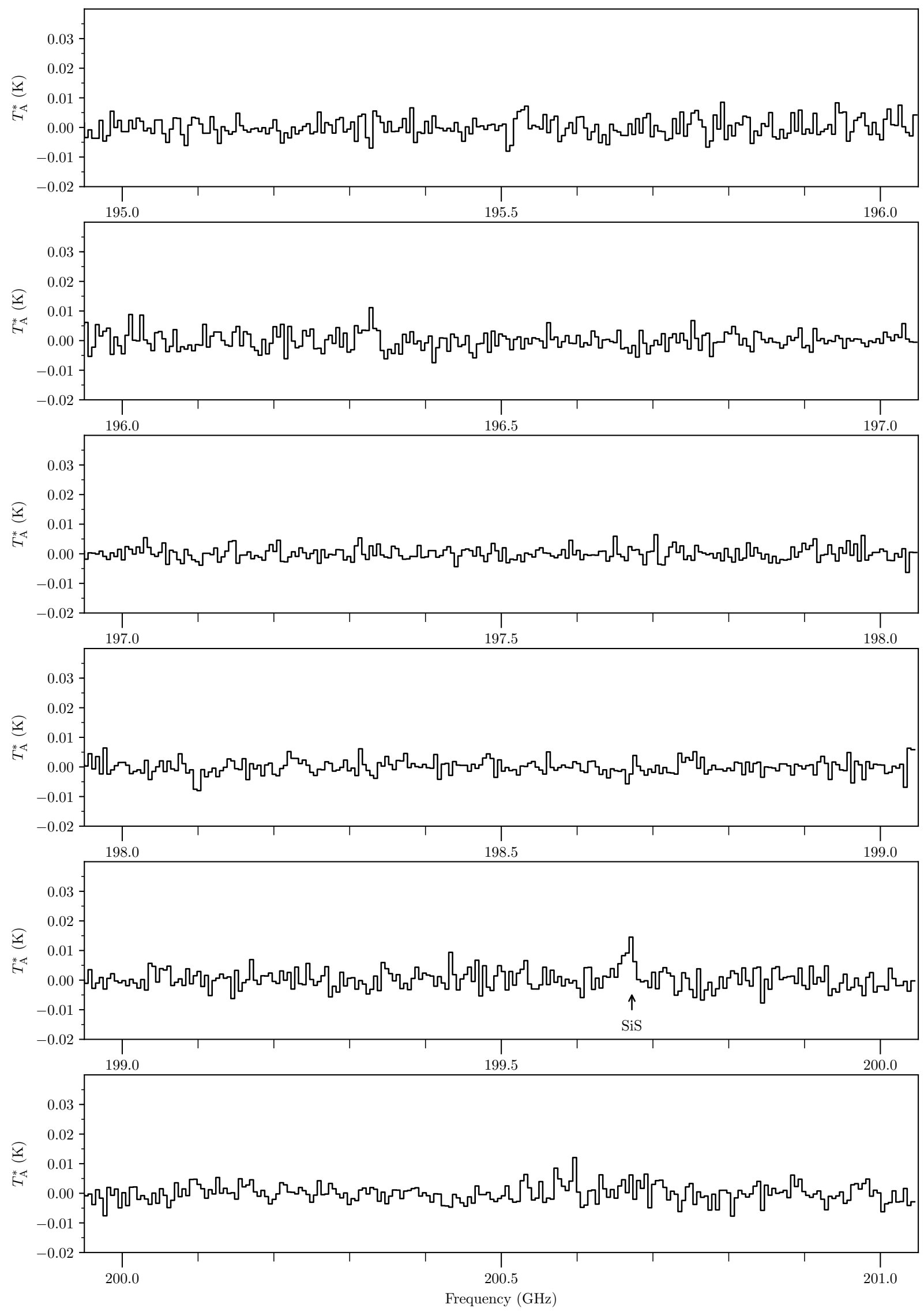

Fig. A.1. continued. 
E. De Beck and H. Olofsson: The surprisingly carbon-rich environment of the S-type star W Aq1
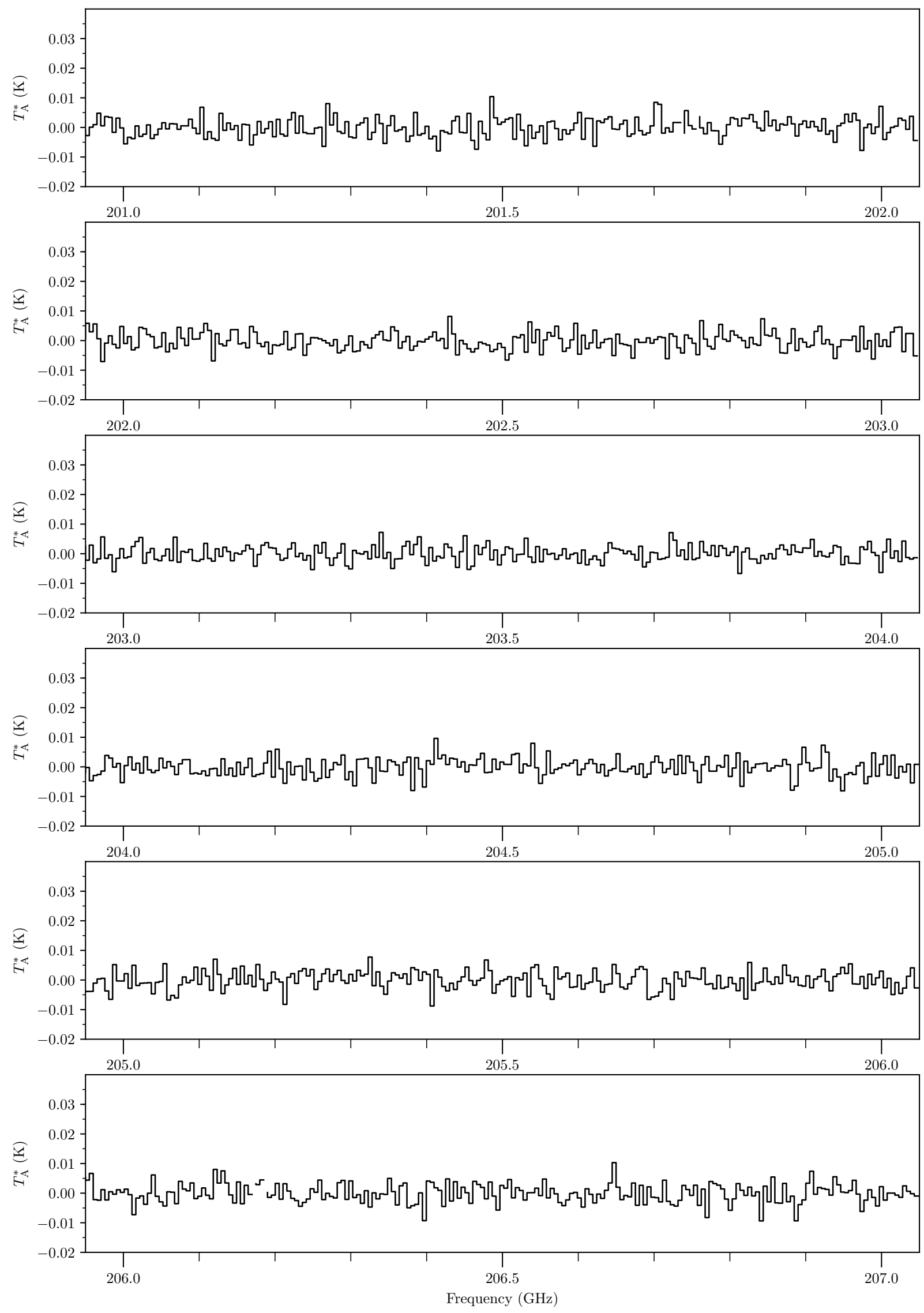

Fig. A.1. continued. 

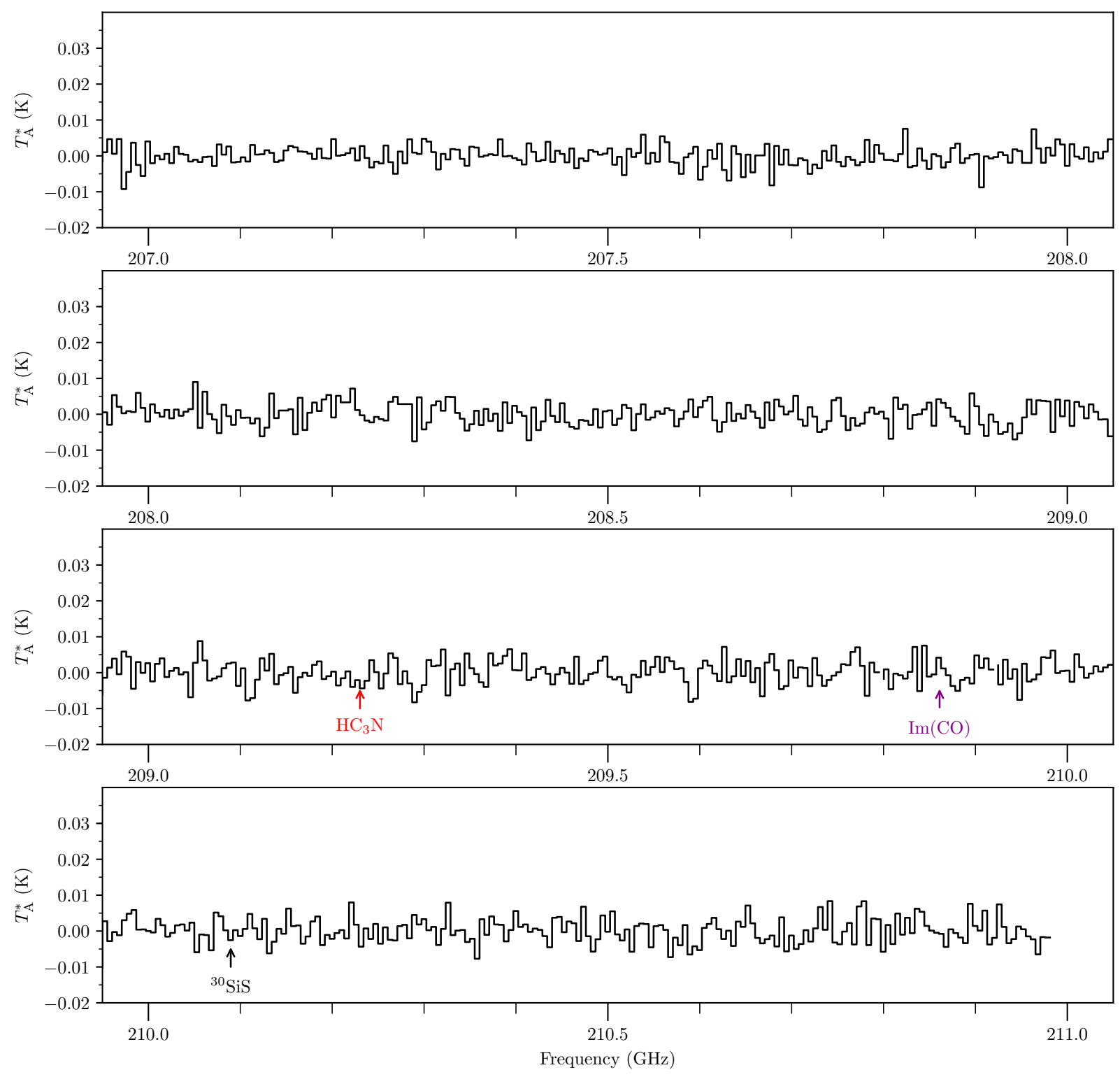

Fig. A.1. continued. 

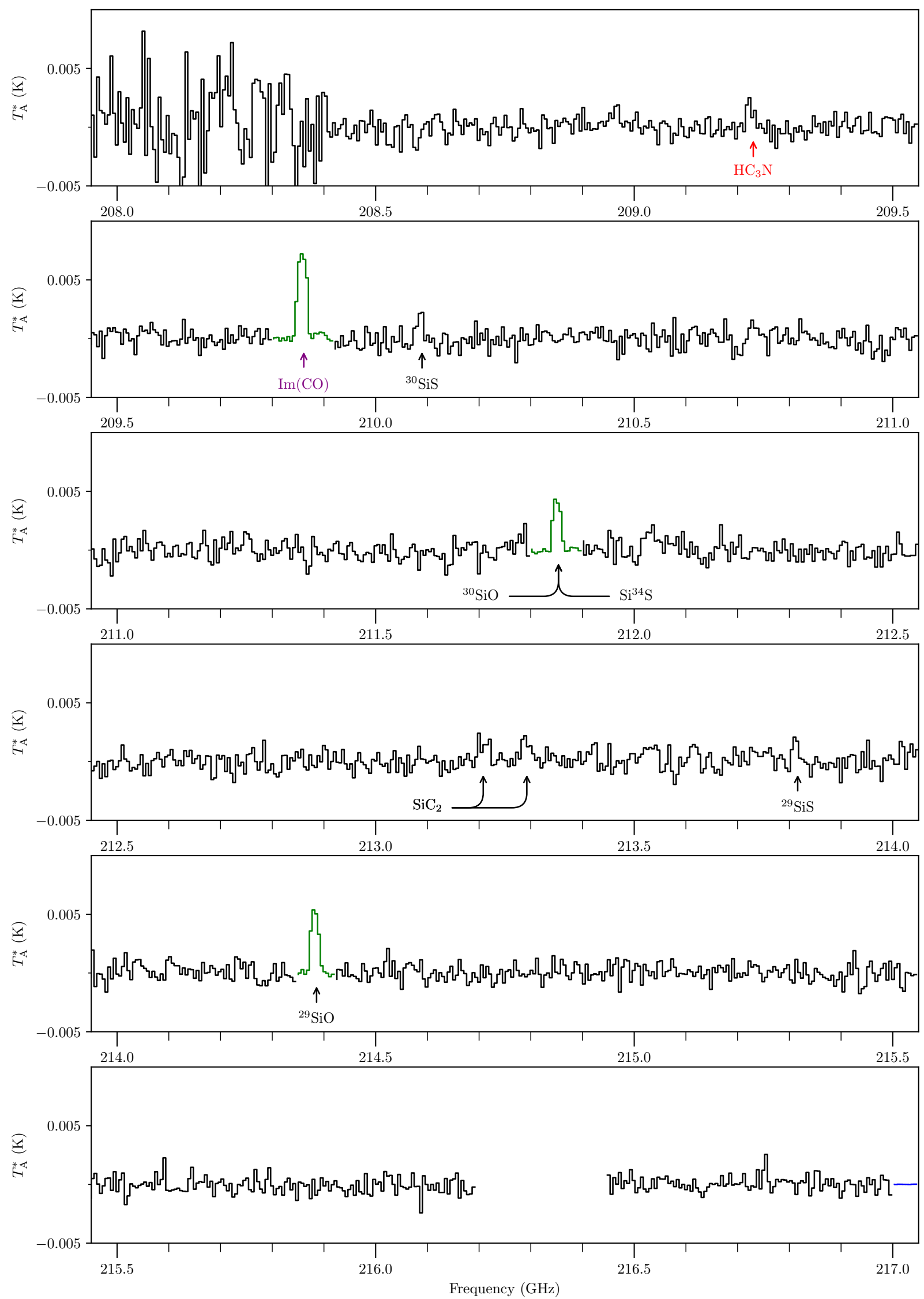

Fig. A.2. APEX line survey of W Aql. Labels show the carrier molecule of the indicated emission. Red labels indicate tentative or unidentified detections. Purple labels $(\operatorname{Im}(. .)$.$) pertain to emission contaminating the signal from the image sideband (see Sect. 2). For visibility, some parts of$ the survey were rescaled to fit the vertical scale. The colour coding of the spectrum corresponds to the following scale factors: (black) 1 ; (green) 1/5; (blue) 1/25; (magenta) 1/125; (orange) 1/250. 

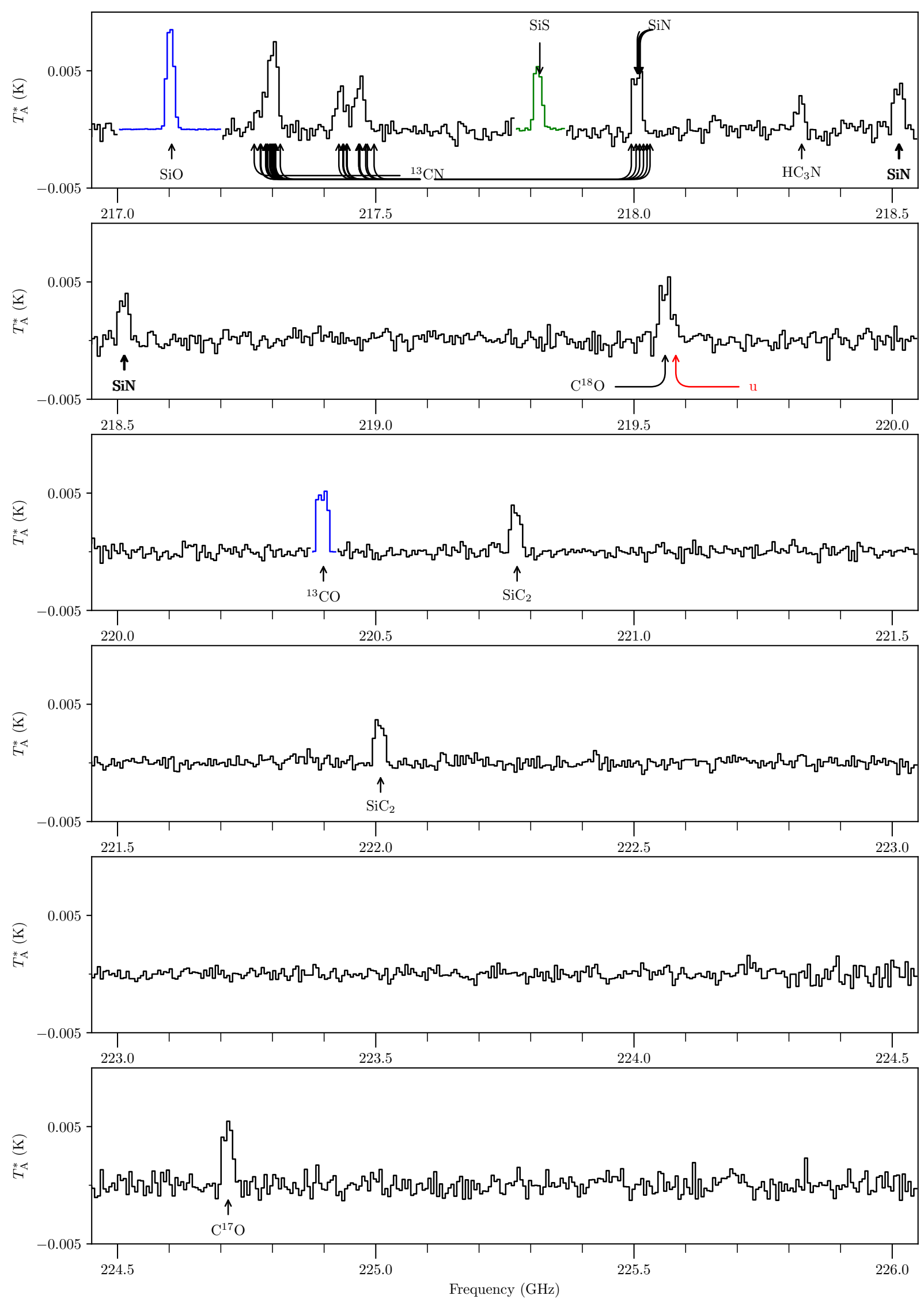

Fig. A.2. continued. 
E. De Beck and H. Olofsson: The surprisingly carbon-rich environment of the S-type star W Aq1
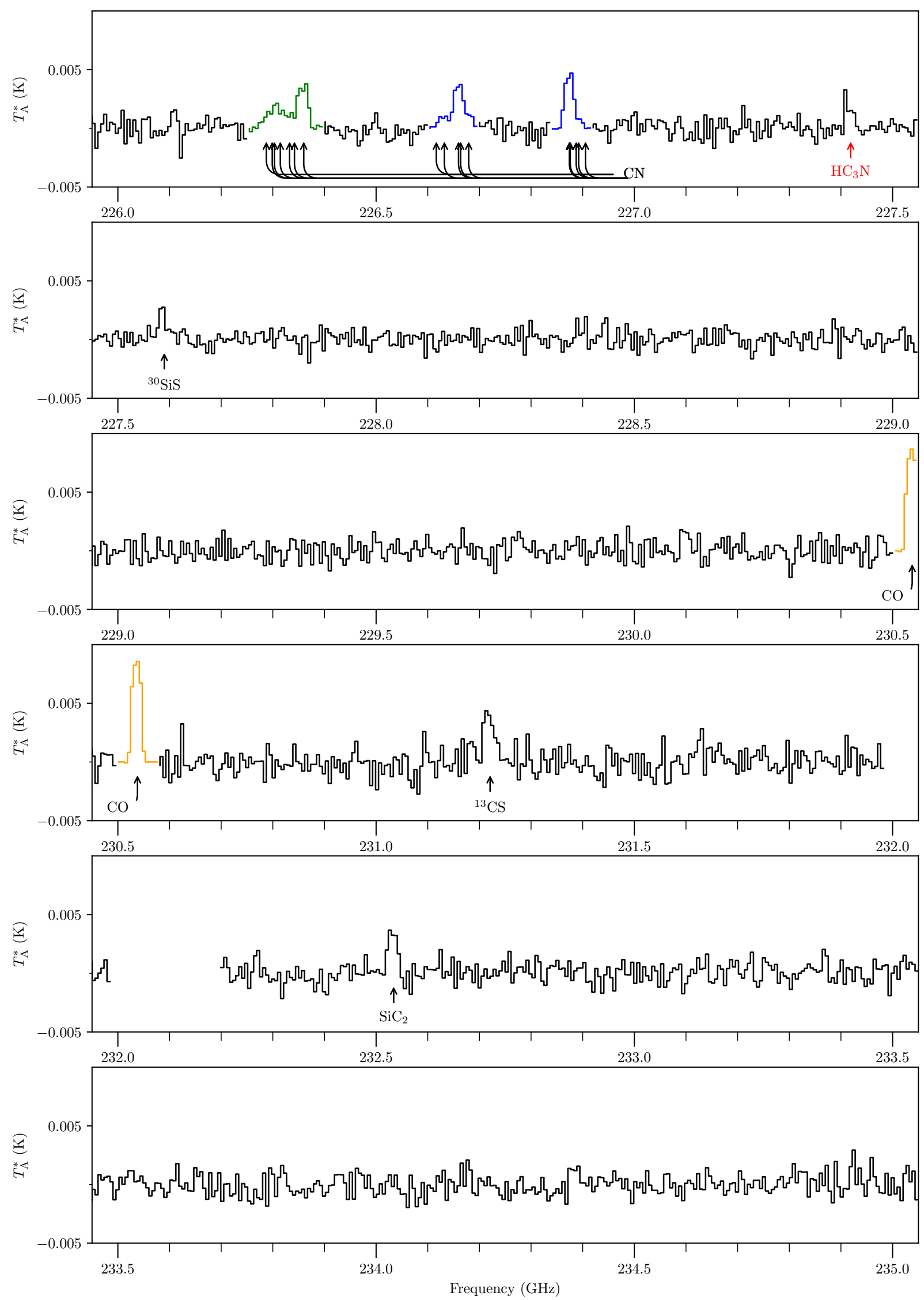

Fig. A.2. continued. 
A\&A 642, A20 (2020)
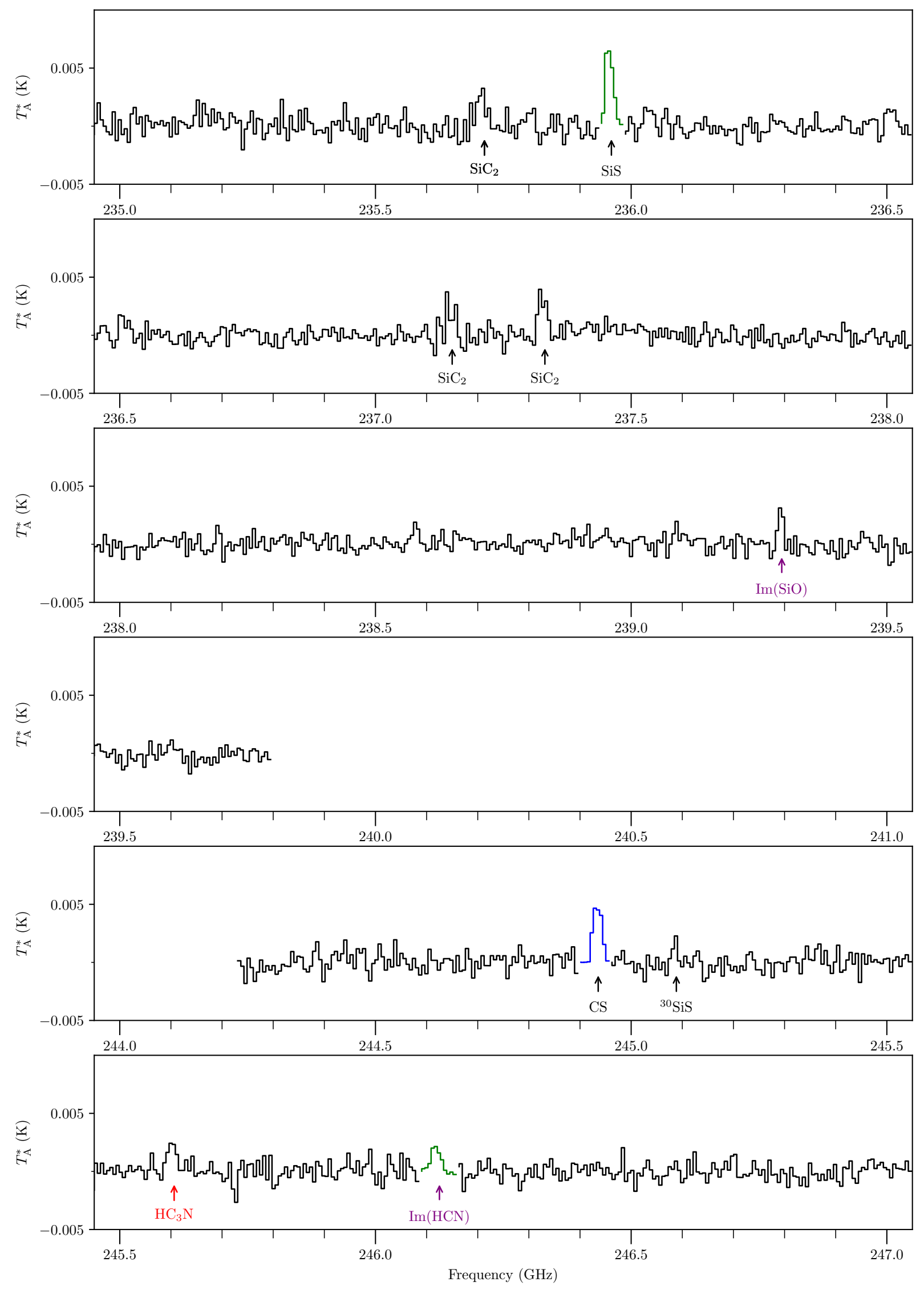

Fig. A.2. continued.

A20, page 26 of 34 
E. De Beck and H. Olofsson: The surprisingly carbon-rich environment of the S-type star W Aq1
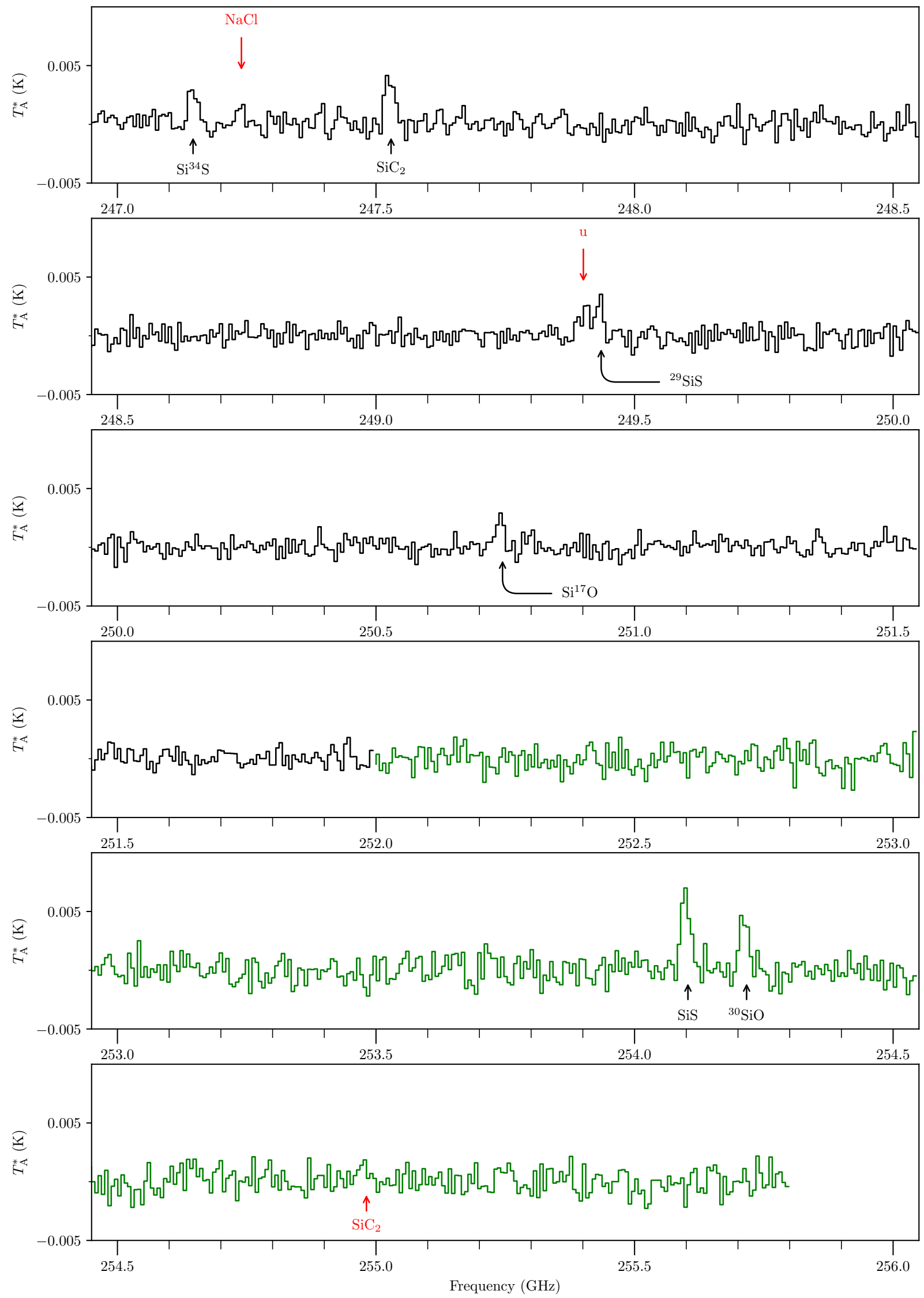

Fig. A.2. continued. 

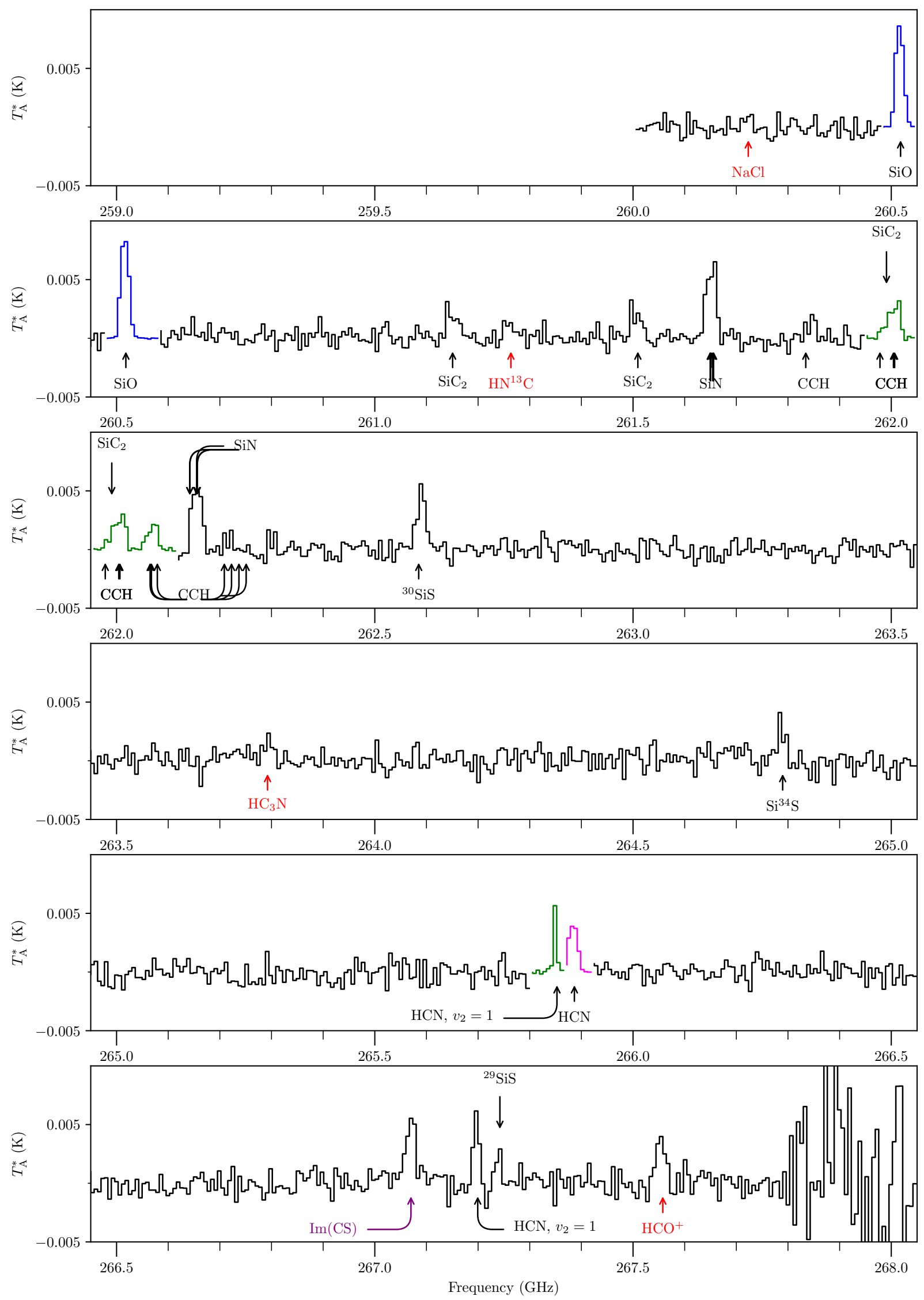

Fig. A.2. continued. 
E. De Beck and H. Olofsson: The surprisingly carbon-rich environment of the S-type star W Aq1

Table A.2. Molecular lines detected in our survey towards W Aql.

\begin{tabular}{|c|c|c|c|c|c|c|c|c|c|}
\hline No. & Molecule & Transition & $\begin{array}{r}E_{\mathrm{u}} / k \\
(\mathrm{~K}) \\
\end{array}$ & $\begin{array}{l}\text { Freq. } \\
(\mathrm{MHz})\end{array}$ & $\begin{array}{c}\text { Res. } \\
(\mathrm{MHz})\end{array}$ & $\begin{array}{c}v_{\min } \\
\left(\mathrm{km} \mathrm{s}^{-1}\right)\end{array}$ & $\begin{array}{c}v_{\max } \\
\left(\mathrm{km} \mathrm{s}^{-1}\right)\end{array}$ & $\begin{array}{r}I_{\text {int }} \\
\left(\mathrm{mK} \mathrm{km} \mathrm{s}^{-1}\right) \\
\end{array}$ & Comments \\
\hline 1 & $\operatorname{Im}(\mathrm{SiO})$ & l & - & 160292.000 & - & - & - & - & Image contamination (Table A.1). \\
\hline 2 & $\operatorname{SiS}(\mathrm{t})$ & $9-8$ & 39.2 & 163376.781 & 3.1 & -25.9 & 31.3 & $192.2 \pm 15.4$ & Tentative. \\
\hline 3 & ${ }^{30} \mathrm{SiO}(\mathrm{t})$ & $4-3$ & 20.3 & 169486.875 & 3.1 & -17.2 & 32.0 & $254.8 \pm 25.4$ & Tentative. \\
\hline 4 & ${ }^{29} \mathrm{SiO}$ & $4-3$ & 20.6 & 171512.797 & 3.1 & -38.5 & 25.0 & $295.2 \pm 45.8$ & \\
\hline 5 & $\mathrm{H}^{13} \mathrm{CN}$ & $2-1$ & 12.4 & 172677.844 & 1.5 & -22.7 & 22.8 & $543.9 \pm 75.6$ & \\
\hline 6 & $\mathrm{HC}_{3} \mathrm{~N}(\mathrm{t})$ & $19-18$ & 83.0 & 172849.297 & 2.3 & -16.7 & 7.5 & $72.6 \pm 11.9$ & Tentative. \\
\hline 7 & $\mathrm{SiO}$ & $4-3$ & 20.8 & 173688.234 & 1.5 & -19.3 & 19.2 & $2313.0 \pm 179.0$ & \\
\hline 8 & $\mathrm{C}_{2} \mathrm{H}(\mathrm{t})$ & $N=2-1, J=5 / 2-3 / 2$ & 12.6 & 174664.000 & 6.1 & -65.9 & 61.1 & $141.0 \pm 61.1$ & Hyperfine structure. Tentative. \\
\hline 9 & $\mathrm{C}_{2} \mathrm{H}(\mathrm{t})$ & $N=2-1, J=3 / 2-1 / 2$ & 12.6 & 174725.000 & 6.1 & -54.6 & 38.8 & $68.9 \pm 35.2$ & Hyperfine structure. Tentative. \\
\hline 10 & $\mathrm{HCN}$ & $2-1$ & 12.8 & 177261.109 & 0.8 & -31.1 & 21.2 & $3005.2 \pm 321.9$ & \\
\hline 11 & $\mathrm{HCO}^{+}(\mathrm{t})$ & $2-1$ & 12.8 & 178375.062 & 2.8 & -26.1 & 26.7 & $47.5 \pm 11.6$ & Tentative. \\
\hline 12 & $C^{34} S(t)$ & $4-3$ & 23.1 & 192818.453 & 3.8 & -18.1 & 17.0 & $163.8 \pm 30.4$ & Tentative. \\
\hline 13 & $\mathrm{SiS}$ & $11-10$ & 57.5 & 199672.234 & 3.1 & -14.4 & 28.6 & $344.1 \pm 48.0$ & Asymmetric. \\
\hline 14 & $\mathrm{HC}_{3} \mathrm{~N}(\mathrm{t})$ & $23-22$ & 120.5 & 209230.234 & 3.7 & -26.3 & 30.6 & $49.3 \pm 5.9$ & Tentative. \\
\hline 15 & $\operatorname{Im}(\mathrm{CO})$ & I & - & 209861.000 & - & - & - & - & Image contamination (Table A.1). \\
\hline 16 & ${ }^{30} \mathrm{SiS}$ & $12-11$ & 65.5 & 210089.625 & 4.5 & -15.2 & 17.9 & $41.9 \pm 4.2$ & \\
\hline 17 & ${ }^{30} \mathrm{SiO}$ & $5-4$ & 30.5 & 211853.469 & 1.5 & -23.7 & 16.8 & $561.1 \pm 60.5$ & Blend with $\mathrm{Si}^{34} \mathrm{~S}$. \\
\hline 18 & $\mathrm{Si}^{34} \mathrm{~S}$ & $12-11$ & 66.1 & 211853.734 & 1.5 & -23.3 & 17.2 & $557.1 \pm 64.1$ & Blend with ${ }^{30} \mathrm{SiO}$. \\
\hline 19 & $\mathrm{SiC}_{2}$ & $9_{4,6}-8_{4,5}$ & 82.3 & 213208.031 & 4.6 & -26.4 & 19.0 & $62.5 \pm 9.4$ & \\
\hline 20 & $\mathrm{SiC}_{2}$ & $9_{4,5}-84,4$ & 82.3 & 213292.344 & 4.6 & -36.3 & 29.2 & $65.1 \pm 5.4$ & \\
\hline 21 & ${ }^{29} \mathrm{SiS}$ & $12-11$ & 66.7 & 213816.141 & 3.1 & -16.3 & 13.6 & $31.3 \pm 10.1$ & \\
\hline 22 & ${ }^{29} \mathrm{SiO}$ & $5-4$ & 30.9 & 214385.750 & 1.5 & -35.7 & 20.8 & $680.1 \pm 49.7$ & \\
\hline 23 & $\mathrm{SiO}$ & $5-4$ & 31.3 & 217104.922 & 0.4 & -31.0 & 26.9 & $5226.6 \pm 124.5$ & \\
\hline 24 & ${ }^{13} \mathrm{CN}$ & $N=2-1, J=3 / 2-1 / 2$ & 15.7 & 217280.000 & 1.5 & -71.5 & 28.3 & $283.2 \pm 63.3$ & Hyperfine structure. \\
\hline 25 & ${ }^{13} \mathrm{CN}$ & $N=2-1, J=5 / 2-3 / 2$ & 15.7 & 217405.000 & 1.8 & -64.1 & -10.1 & $101.2 \pm 35.4$ & Hyperfine structure. \\
\hline 26 & ${ }^{13} \mathrm{CN}$ & $N=2-1, J=5 / 2-3 / 2$ & 15.7 & 217469.000 & 1.8 & -42.0 & 17.6 & $126.6 \pm 39.3$ & Hyperfine structure. \\
\hline 27 & $\mathrm{SiS}$ & $12-11$ & 68.0 & 217817.656 & 1.5 & -49.6 & 32.8 & $674.9 \pm 54.8$ & \\
\hline 28 & $\mathrm{SiN}$ & $N=5-4, J=9 / 2-7 / 2$ & 31.4 & 218008.000 & 1.5 & -20.5 & 16.9 & $134.9 \pm 35.5$ & \\
\hline 29 & $\mathrm{HC}_{3} \mathrm{~N}(\mathrm{t})$ & $24-23$ & 131.0 & 218324.719 & 1.8 & -17.7 & 18.3 & $62.4 \pm 12.6$ & Tentative. \\
\hline 30 & SiN & $N=5-4, J=11 / 2-9 / 2$ & 31.5 & 218513.000 & 1.5 & -20.7 & 16.5 & $112.6 \pm 29.7$ & \\
\hline 31 & $\mathrm{C}^{18} \mathrm{O}$ & $2-1$ & 15.8 & 219560.359 & 1.5 & -20.1 & 15.9 & $143.1 \pm 44.9$ & Blend with unidentified feature. \\
\hline 32 & $\mathrm{u}$ & 1 & - & 219581.000 & 1.8 & -18.2 & 43.6 & $186.3 \pm 89.5$ & No candidate. Blend with $\mathrm{C}^{18} \mathrm{O}$. \\
\hline 33 & ${ }^{13} \mathrm{CO}$ & $2-1$ & 15.9 & 220398.688 & 0.4 & -32.5 & 28.7 & $4182.7 \pm 114.8$ & \\
\hline 34 & $\mathrm{SiC}_{2}$ & $10_{0,10}-9_{0,9}$ & 59.8 & 220773.688 & 1.5 & -31.4 & 17.4 & $106.8 \pm 26.1$ & \\
\hline 35 & $\mathrm{SiC}_{2}$ & $9_{2,7}-82,6$ & 60.2 & 222009.391 & 1.5 & -25.7 & 14.6 & $106.6 \pm 28.1$ & \\
\hline 36 & $\mathrm{C}^{17} \mathrm{O}$ & $2-1$ & 16.2 & 224714.203 & 2.3 & -35.9 & 16.2 & $154.6 \pm 31.6$ & \\
\hline 37 & $\mathrm{CN}$ & $N=2-1, J=3 / 2-3 / 2$ & 16.3 & 226310.000 & 1.5 & -89.8 & 46.9 & $1145.8 \pm 208.0$ & Hyperfine structure. \\
\hline 38 & $\mathrm{CN}$ & $N=2-1, J=3 / 2-1 / 2$ & 16.3 & 226659.000 & 1.5 & -54.0 & 77.8 & $4099.5 \pm 182.0$ & Hyperfine structure. \\
\hline 39 & $\mathrm{CN}$ & $N=2-1, J=5 / 2-3 / 2$ & 16.3 & 226876.000 & 1.5 & -45.1 & 33.6 & $3513.1 \pm 117.0$ & Hyperfine structure. \\
\hline 40 & $\mathrm{HC}_{3} \mathrm{~N}(\mathrm{t})$ & $25-24$ & 141.9 & 227418.906 & 3.7 & -19.4 & 18.6 & $56.2 \pm 9.9$ & Tentative. \\
\hline 41 & ${ }^{30} \mathrm{SiS}$ & $13-12$ & 76.5 & 227589.859 & 3.1 & -26.0 & 14.6 & $60.8 \pm 12.8$ & \\
\hline 42 & $\mathrm{CO}$ & $2-1$ & 16.6 & 230538.000 & 0.4 & -37.6 & 24.2 & $58918.6 \pm 1654.7$ & \\
\hline 43 & ${ }^{13} \mathrm{CS}$ & $5-4$ & 33.3 & 231220.688 & 3.1 & -20.0 & 21.6 & $127.1 \pm 18.1$ & \\
\hline 44 & $\mathrm{SiC}_{2}$ & $10_{2,9}-9_{2,8}$ & 69.6 & 232534.062 & 1.9 & -28.7 & 16.7 & $100.8 \pm 32.6$ & \\
\hline 45 & $\mathrm{SiC}_{2}$ & $10_{6,5}-9_{6,4}$ & 132.5 & 235713.000 & 4.8 & -22.9 & 23.4 & $56.6 \pm 17.8$ & Doublet blend. \\
\hline 46 & $\mathrm{SiC}_{2}$ & $10_{6,4}-9_{6,3}$ & 132.5 & 235713.062 & 4.8 & -22.8 & 23.5 & $56.6 \pm 17.8$ & Doublet blend. \\
\hline 47 & $\mathrm{SiS}$ & $13-12$ & 79.3 & 235961.359 & 1.5 & -33.0 & 31.5 & $832.0 \pm 112.2$ & Irregular shape. \\
\hline 48 & $\mathrm{HC}_{3} \mathrm{~N}(\mathrm{t})$ & $26-25$ & 153.2 & 236512.781 & 2.3 & -22.6 & 24.7 & $41.2 \pm 24.1$ & Tentative. \\
\hline 49 & $\mathrm{SiC}_{2}$ & $10_{4,7}-9_{4,6}$ & 93.7 & 237150.016 & 4.2 & -24.6 & 19.1 & $50.5 \pm 18.2$ & \\
\hline 50 & $\mathrm{SiC}_{2}$ & $10_{4,6}-94,5$ & 93.7 & 237331.312 & 4.2 & -20.3 & 21.2 & $90.4 \pm 15.2$ & \\
\hline 51 & $\operatorname{Im}(\mathrm{SiO})$ & 1 & - & 239295.000 & - & - & - & - & Image contamination (Table A.1). \\
\hline 52 & $\mathrm{CS}$ & $5-4$ & 35.3 & 244935.562 & 0.8 & -33.6 & 19.9 & $3272.4 \pm 230.3$ & \\
\hline 53 & ${ }^{30} \mathrm{SiS}(\mathrm{t})$ & $14-13$ & 88.2 & 245088.391 & 4.6 & -12.6 & 15.9 & $28.2 \pm 5.3$ & Tentative. \\
\hline 54 & $\mathrm{HC}_{3} \mathrm{~N}(\mathrm{t})$ & $27-26$ & 165.0 & 245606.312 & 2.4 & -15.7 & 24.4 & $63.6 \pm 15.4$ & Tentative. \\
\hline 55 & $\operatorname{Im}(\mathrm{HCN})$ & l & - & 246125.000 & - & - & - & - & Image contamination (Table A.1). \\
\hline 56 & $\mathrm{Si}^{34} \mathrm{~S}$ & $14-13$ & 89.0 & 247146.234 & 3.1 & -32.4 & 17.0 & $77.3 \pm 9.3$ & \\
\hline 57 & $\mathrm{NaCl}(\mathrm{t})$ & $19-18$ & 118.7 & 247239.734 & 4.9 & -49.2 & 63.1 & $28.9 \pm 25.5$ & Tentative. \\
\hline 58 & $\mathrm{SiC}_{2}$ & $10_{2,8}-9_{2,7}$ & 72.1 & 247529.125 & 4.2 & -18.9 & 18.0 & $110.2 \pm 15.7$ & \\
\hline 59 & $\mathrm{u}$ & 1 & - & 249401.000 & 2.4 & -58.9 & 20.3 & $132.8 \pm 88.8$ & No candidate. \\
\hline 60 & ${ }^{29} \mathrm{SiS}$ & $14-13$ & 89.8 & 249435.406 & 2.4 & -15.4 & 14.7 & $65.7 \pm 7.6$ & \\
\hline 61 & $\mathrm{Si}^{17} \mathrm{O}$ & $6-5$ & 42.1 & 250744.688 & 4.9 & -13.4 & 23.1 & $58.9 \pm 2.7$ & \\
\hline 62 & $\mathrm{SiS}$ & $14-13$ & 91.5 & 254103.219 & 2.3 & -31.7 & 18.0 & $887.1 \pm 190.1$ & \\
\hline 63 & ${ }^{30} \mathrm{SiO}$ & $6-5$ & 42.7 & 254216.656 & 3.1 & -18.4 & 17.5 & $491.9 \pm 61.0$ & \\
\hline 64 & $\mathrm{SiC}_{2}(\mathrm{t})$ & $11_{2,10}-10_{2,9}$ & 81.9 & 254981.500 & 5.3 & -21.8 & 27.7 & $215.7 \pm 92.9$ & Tentative. \\
\hline 65 & $\mathrm{NaCl}(\mathrm{t})$ & $20-19$ & 131.2 & 260223.109 & 4.9 & -46.8 & 65.8 & $5.3 \pm 7.1$ & Tentative. \\
\hline
\end{tabular}


Table A.2. continued.

\begin{tabular}{|c|c|c|c|c|c|c|c|c|c|}
\hline No. & Molecule & Transition & $\begin{array}{r}E_{\mathrm{u}} / k \\
(\mathrm{~K})\end{array}$ & $\begin{array}{c}\text { Freq. } \\
(\mathrm{MHz})\end{array}$ & $\begin{array}{l}\text { Res. } \\
(\mathrm{MHz})\end{array}$ & $\begin{array}{c}v_{\min } \\
\left(\mathrm{km} \mathrm{s}^{-1}\right)\end{array}$ & $\begin{array}{c}v_{\max } \\
\left(\mathrm{km} \mathrm{s}^{-1}\right)\end{array}$ & $\left(\mathrm{mK} \mathrm{km} \mathrm{s}^{-1}\right)$ & Comments \\
\hline 66 & $\mathrm{SiO}$ & $6-5$ & 43.8 & 260518.016 & 1.5 & -28.1 & 28.7 & $4905.4 \pm 280.9$ & \\
\hline 67 & $\mathrm{SiC}_{2}$ & $11_{4,8}-10_{4,7}$ & 106.2 & 261150.688 & 5.3 & -24.8 & 24.3 & $67.0 \pm 10.3$ & \\
\hline 68 & $\mathrm{HN}^{13} \mathrm{C}$ & $3-2$ & 25.1 & 261263.516 & 4.9 & -39.5 & 21.4 & $39.2 \pm 12.6$ & Tentative. \\
\hline 69 & $\mathrm{SiC}_{2}$ & $11_{4,7}-10_{4,6}$ & 106.2 & 261509.328 & 3.8 & -23.0 & 21.7 & $59.7 \pm 13.7$ & \\
\hline 70 & $\mathrm{SiN}$ & $N=6-5, J=11 / 2-9 / 2$ & 43.9 & 261650.000 & 1.5 & -21.5 & 14.6 & $162.5 \pm 35.6$ & \\
\hline 71 & $\mathrm{SiC}_{2}$ & $12_{0,12}-11_{0,11}$ & 83.9 & 261990.750 & 2.3 & -16.3 & 18.5 & $200.5 \pm 29.7$ & Blend with $\mathrm{C}_{2} \mathrm{H}$. \\
\hline 72 & $\mathrm{C}_{2} \mathrm{H}$ & $N=3-2, J=7 / 2-5 / 2$ & 25.1 & 262005.000 & 2.3 & -27.9 & 37.2 & $490.4 \pm 47.2$ & Hyperfine structure. Blend with $\mathrm{SiC}_{2}$ \\
\hline 73 & $\mathrm{C}_{2} \mathrm{H}$ & $N=3-2, J=5 / 2-3 / 2$ & 25.2 & 262067.000 & 2.3 & -54.0 & 21.3 & $294.7 \pm 23.6$ & Hyperfine structure. \\
\hline 74 & $\mathrm{SiN}$ & $N=6-5, J=13 / 2-11 / 2$ & 44.1 & 262155.000 & 2.3 & -27.0 & 13.3 & $150.4 \pm 27.0$ & \\
\hline 75 & $\mathrm{C}_{2} \mathrm{H}(\mathrm{t})$ & $N=3-2, J=5 / 2-5 / 2$ & 25.2 & 262208.000 & 6.9 & -63.7 & 8.6 & $21.6 \pm 10.9$ & Hyperfine structure. Tentative. \\
\hline 76 & ${ }^{30} \mathrm{SiS}$ & $15-14$ & 100.8 & 262585.031 & 3.1 & -30.9 & 12.1 & $117.1 \pm 20.2$ & Asymmetric. \\
\hline 77 & $\mathrm{HC}_{3} \mathrm{~N}(\mathrm{t})$ & $29-28$ & 189.9 & 263792.312 & 4.9 & -22.9 & 18.0 & $41.3 \pm 8.3$ & Tentative. \\
\hline 78 & $\mathrm{Si}^{34} \mathrm{~S}$ & $15-14$ & 101.7 & 264789.719 & 3.1 & -15.7 & 9.0 & $60.2 \pm 13.2$ & \\
\hline 79 & $\mathrm{HCN}, v_{2}=1$ & $3_{1}-2_{-1}$ & 1049.9 & 265852.719 & 1.5 & -12.4 & 19.3 & $272.5 \pm 13.8$ & \\
\hline 80 & $\mathrm{HCN}$ & $3-2$ & 25.5 & 265886.438 & 1.5 & -33.8 & 25.7 & $12283.4 \pm 465.7$ & \\
\hline 81 & $\operatorname{Im}(\mathrm{CS})$ & / & - & 267070.000 & - & - & - & - & Image contamination (Table A.1). \\
\hline 82 & $\mathrm{HCN}, v_{2}=1$ & $3_{-1}-2_{1}$ & 1050.0 & 267199.281 & 3.1 & -17.0 & 15.4 & $97.5 \pm 9.9$ & \\
\hline 83 & ${ }^{29} \mathrm{SiS}$ & $15-14$ & 102.6 & 267242.219 & 3.1 & -8.0 & 15.8 & $41.6 \pm 4.6$ & \\
\hline 84 & $\mathrm{HCO}^{+}(\mathrm{t})$ & $3-2$ & 25.7 & 267557.625 & 2.4 & -26.0 & 28.7 & $95.4 \pm 29.0$ & Tentative. \\
\hline
\end{tabular}

\section{Appendix B: Detections}

This appendix features all line emission detected in the spectra in Appendix A other than the lines presented in the body of the paper. In all of these figures the grey-shaded area designates the spectral range specified in Table A.2 and used to calculate the listed integrated intensities.

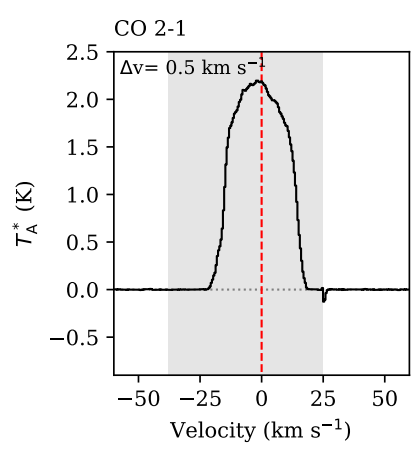

(a) $\mathrm{CO}$

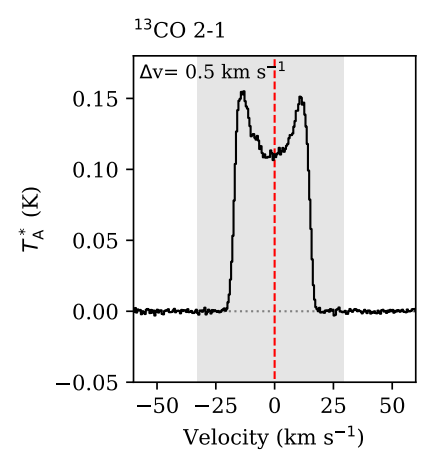

(b) ${ }^{13} \mathrm{CO}$

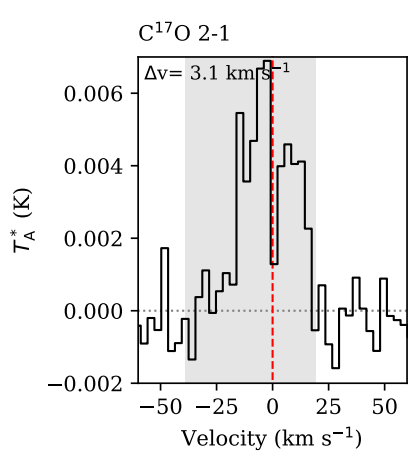

(c) $\mathrm{C}^{17} \mathrm{O}$

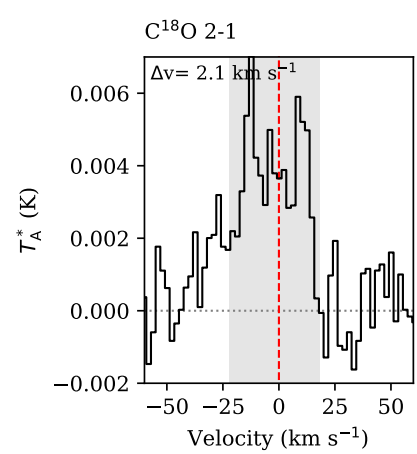

(d) $\mathrm{C}^{18} \mathrm{O}$

Fig. B.1. $\mathrm{CO}$ emission. 
E. De Beck and H. Olofsson: The surprisingly carbon-rich environment of the S-type star W Aq1
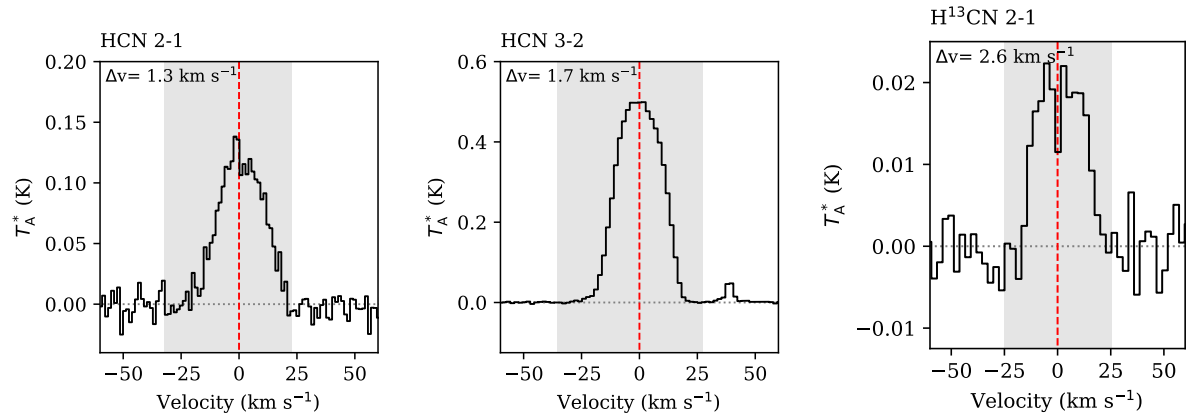

(a) $\mathrm{HCN}$

(b) $\mathrm{H}^{13} \mathrm{CN}$
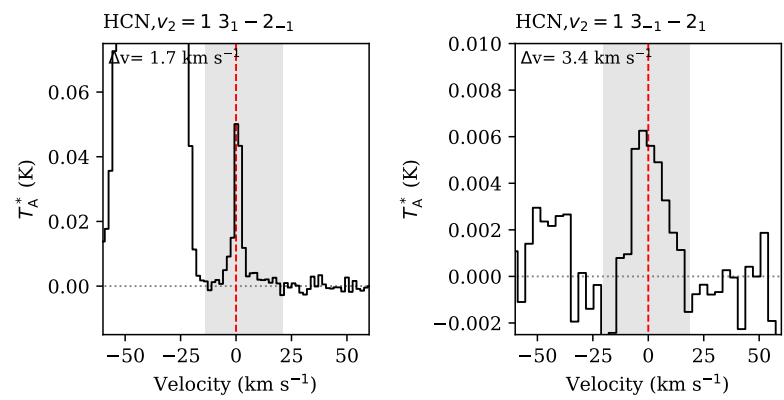

(c) $\mathrm{HCN}, v_{2}=1$

Fig. B.2. HCN emission.
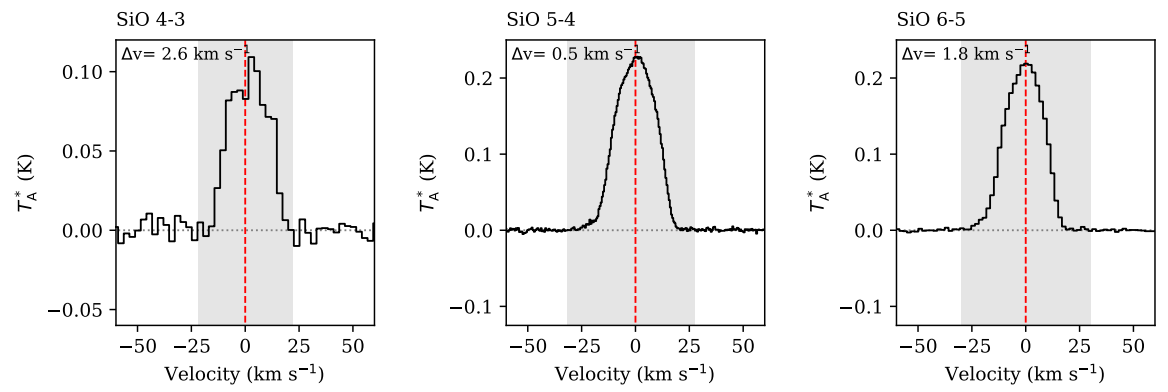

(a) $\mathrm{SiO}$
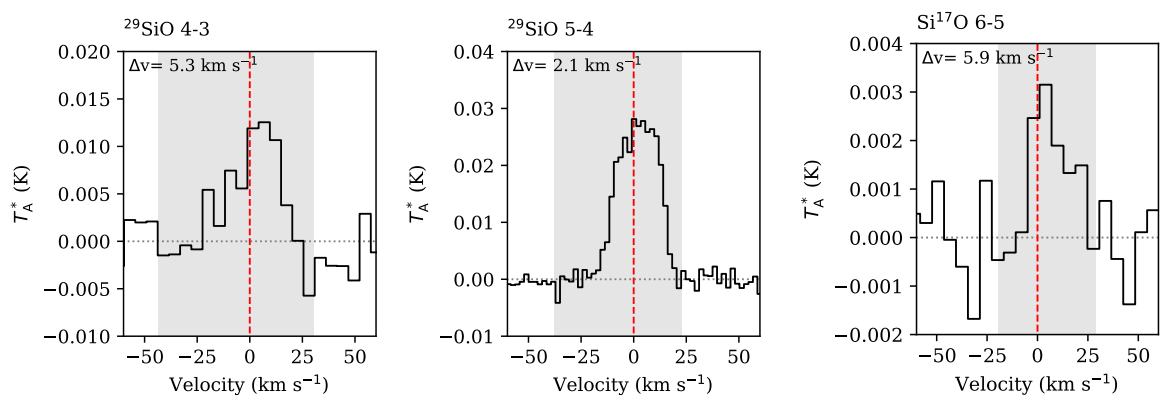

(b) ${ }^{29} \mathrm{SiO}$

(c) $\mathrm{Si}^{17} \mathrm{O}$
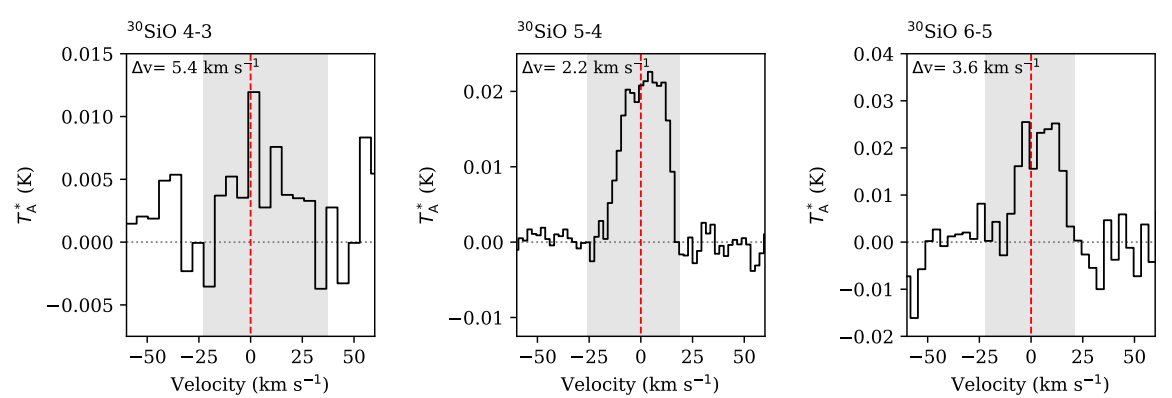

(d) ${ }^{30} \mathrm{SiO}$. Note that the $J=5-4$ emission is blended with emission from $\mathrm{Si}^{34} \mathrm{~S}(J=12-11)$.

Fig. B.3. $\mathrm{SiO}$ emission. 

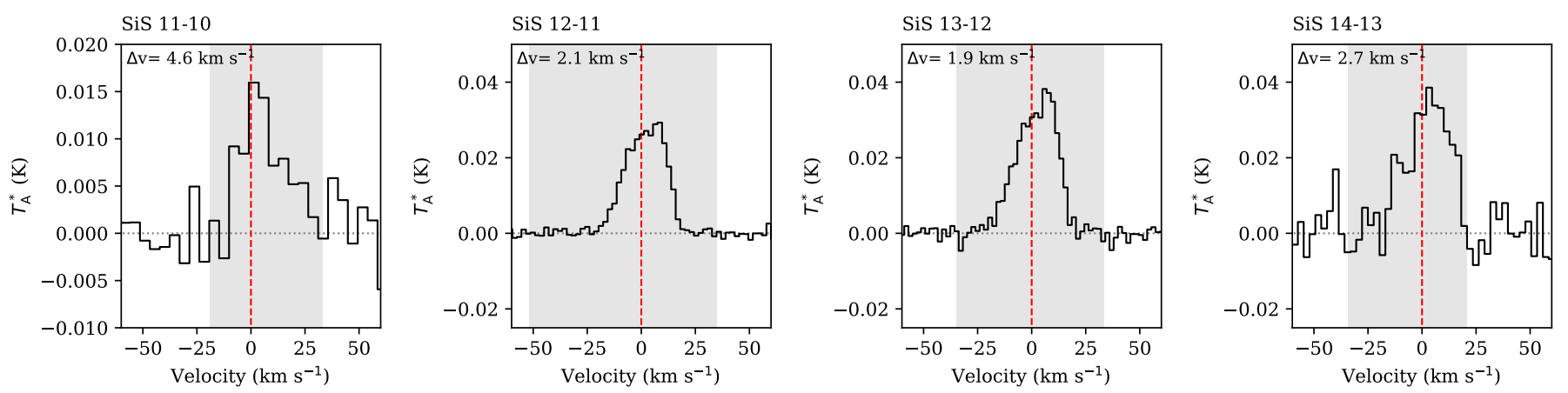

(a) $\mathrm{SiS}$
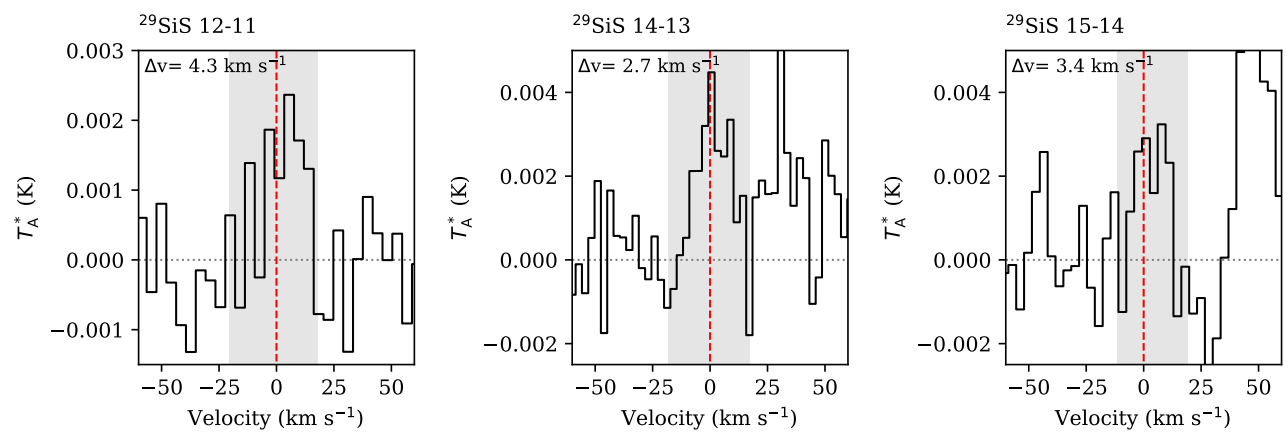

(b) ${ }^{29} \mathrm{SiS}$
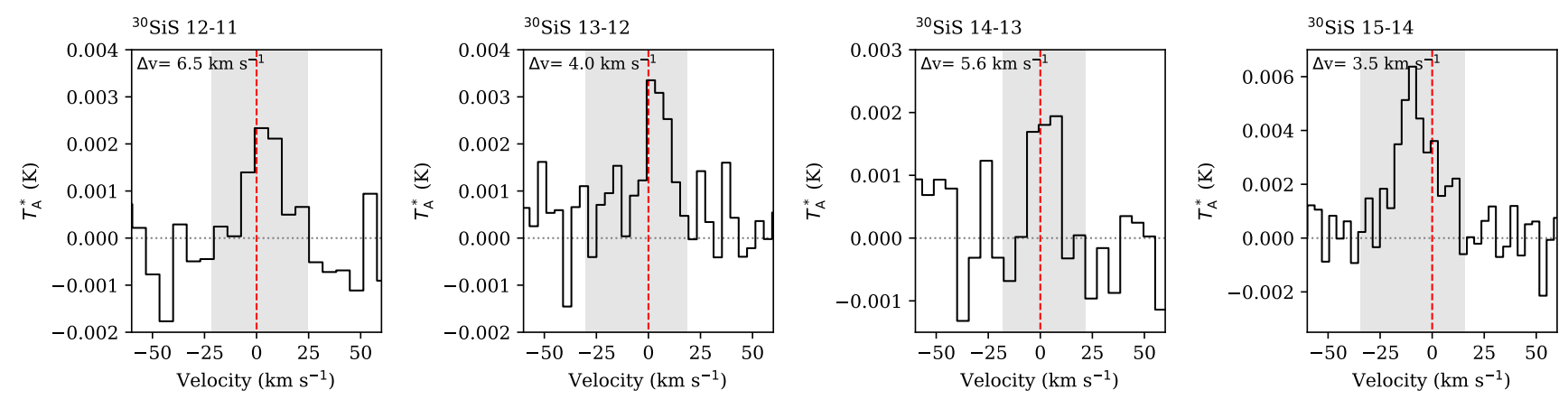

(c) ${ }^{30} \mathrm{SiS}$
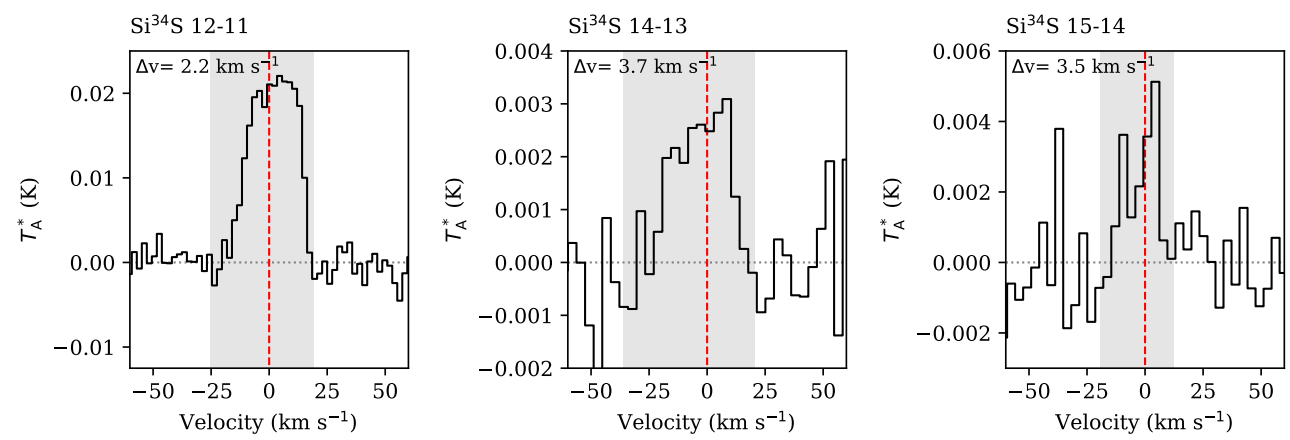

(d) $\mathrm{Si}^{34} \mathrm{~S}$. Note that the $J=12-11$ emission is blended with emission from ${ }^{30} \mathrm{SiO}(J=5-4)$

Fig. B.4. SiS emission 
E. De Beck and H. Olofsson: The surprisingly carbon-rich environment of the S-type star W Aq1

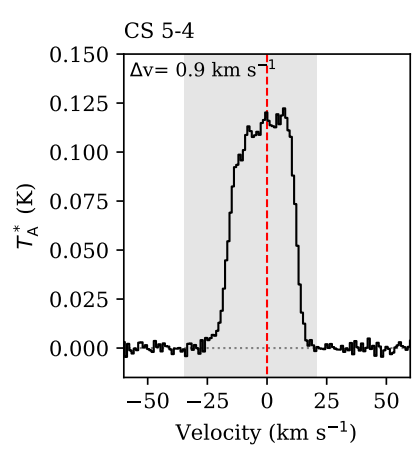

(a) $\mathrm{CS}$

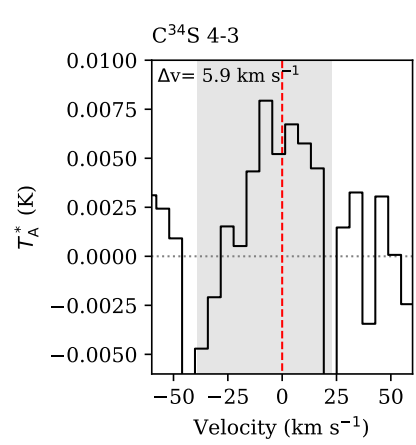

(b) $\mathrm{C}^{34} \mathrm{~S}$

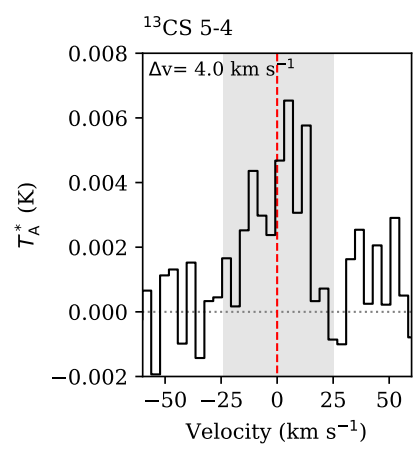

(c) ${ }^{13} \mathrm{CS}$

Fig. B.5. CS emission.
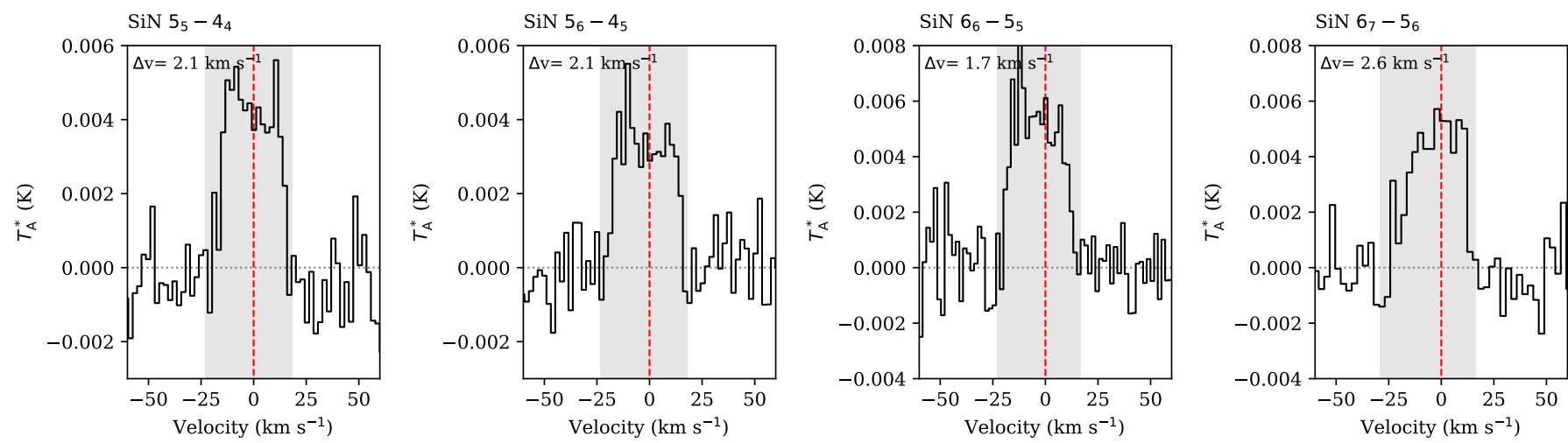

Fig. B.6. SiN emission. Rest frequencies were calculated by weighting the hyperfine component frequencies with the intrinsic component intensities.
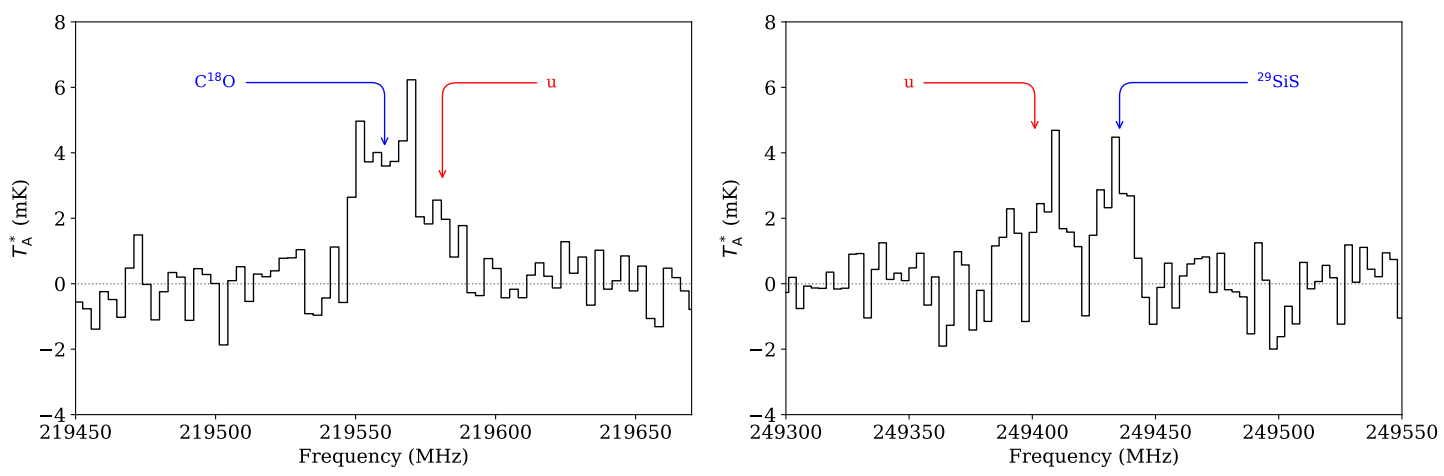

Fig. B.7. Unidentified emission features. 


\section{Appendix C: Fractional abundances for M-, S-, C-type CSEs}

The peak fractional abundances used to compile Fig. 8 are listed in Table C.1, along with the references to the relevant studies and a marker in case of upper limits. We refer to those papers for presentation of and discussion on the modelling procedures used to estimate the abundances and the target samples used to obtain median abundance values for M-type, S-type, and C-type stars.

Table C.1. Peak fractional abundances used in Fig. 8.

\begin{tabular}{|c|c|c|c|c|c|c|c|c|c|}
\hline Molecule & R Dor & IK Tau & M-type & $\chi$ Cyg & W Aql & S-type & V Aql & IRC +10 216 & C-type \\
\hline $\mathrm{HCN}$ & $9.0(-8)^{(1)}$ & $2.2(-7)^{(2)}$ & $1.2(-7)^{(1)}$ & $5.0(-6)^{(3)}$ & $3.1 \pm 0.1(-6)^{(4)}$ & $7.0(-7)^{(1)}$ & $1.3(-5)^{(1)}$ & $2.0(-5)^{(5)}$ & $2.5(-5)^{(1)}$ \\
\hline $\mathrm{CS}$ & - & $4.0(-8)^{(2)}$ & $8.0(-8)^{(6)}$ & $1.0(-7)^{(3)}$ & $1.2 \pm 0.05(-6)^{(7)}$ & $5.0(-7)^{(6)}$ & $1.0(-5)^{(8)}$ & $4.0(-6)^{(5)}$ & $7.0(-6)^{(8)}$ \\
\hline $\mathrm{SiS}$ & - & $5.5(-6)^{(2)}$ & $1.1(-6)^{(6)}$ & $1.8(-7)^{(3)}$ & $1.5 \pm 0.05(-6)^{(7)}$ & $6.0(-7)^{(6)}$ & $2.3(-6)^{(8)(\dagger)}$ & $3.0(-6)^{(5)}$ & $1.0(-5)^{(8)}$ \\
\hline $\mathrm{SiO}$ & $2.0(-5)^{(9)}$ & $2.0(-7)^{(2)}$ & $5.0(-6)^{(10)}$ & $1.3(-5)^{(3)}$ & $2.9 \pm 0.7(-6)^{(4)}$ & $6.0(-6)^{(11)}$ & $2.5(-6)^{(8)}$ & $1.8(-7)^{(5)}$ & $3.8(-6)^{(12)}$ \\
\hline $\mathrm{H}_{2} \mathrm{O}$ & $2.5(-4)^{(13)}$ & $4.2(-4)$ & - & $1.2(-5)^{(3)}$ & $1.5 \pm 0.5(-5)^{(4)}$ & - & - & $1.0(-7)^{(5)}$ & - \\
\hline $\mathrm{NH}_{3}$ & - & $6.0(-7)^{(14)}$ & - & $1.8(-8)$ & $1.7 \pm 1.0(-5)^{(4)}$ & - & - & $6.0(-8)^{(15)}$ & - \\
\hline $\mathrm{CN}$ & - & $3.0(-8)^{(2)}$ & - & $1.9(-5)^{(3)}$ & $5.7(-6)^{(4)}$ & - & - & $1.7(-6)^{(16)}$ & - \\
\hline $\mathrm{SiC}_{2}$ & - & - & - & - & $5.0 \pm 2.0(-7)$ & - & $2.0(-5)^{(17)}$ & $3.7(-7)^{(17)}$ & $6.3(-6)^{(17)}$ \\
\hline $\mathrm{C}_{2} \mathrm{H}$ & - & - & - & - & $1.0(-5)^{(\ddagger)}$ & - & - & $2.0(-5)^{(18)}$ & - \\
\hline $\mathrm{HNC}$ & - & $8.0(-9)^{(19)}$ & - & - & $3.1(-8)(\ddagger)(*)$ & - & - & $7.0(-8)^{(20)}$ & - \\
\hline $\mathrm{HC}_{3} \mathrm{~N}$ & - & - & - & - & $8.4(-7)^{(\dagger)}$ & - & - & $1.4(-6)^{(16)}$ & - \\
\hline $\mathrm{SiN}$ & - & - & - & - & $4.0(-8)^{(\ddagger)}$ & - & - & $8.0(-9)^{(16)}$ & - \\
\hline $\mathrm{H}_{2} \mathrm{~S}$ & - & $1.6(-6)^{(21)}$ & - & - & $5.0(-7)^{(\dagger)}$ & - & - & $4.0(-9)^{(5)}$ & - \\
\hline $\mathrm{PN}$ & - & $3.0(-7)^{(22)}$ & - & - & $3.0(-7)^{(\dagger)}$ & - & - & $1.0(-9)^{(16)}$ & - \\
\hline $\mathrm{HCO}^{+}$ & - & $1.0(-8)^{(19)}$ & - & - & $7.0(-8)^{(\dagger)}$ & - & - & $4.1(-9)^{(23)}$ & - \\
\hline $\mathrm{NaCl}$ & - & $3.1(-7)^{(19)}$ & - & - & $3.1(-8)^{(\dagger)}$ & - & - & $1.8(-9)^{(5)}$ & - \\
\hline SO & $6.7(-6)^{(24)}$ & $1.0(-6)^{(24)}$ & - & - & $1.0(-6)^{(\dagger)}$ & - & - & - & - \\
\hline $\mathrm{SO}_{2}$ & $5.0(-6)^{(24)}$ & $8.6(-7)^{(24)}$ & - & - & $5.0(-6)^{(\dagger)}$ & - & - & - & - \\
\hline
\end{tabular}

Notes. Fractional abundances $a \times 10^{b}$ are listed as $a(b),(a \pm e) \times 10^{b}$ as $a \pm e(b)$. Where no reference is given, the abundance is estimated in this work. ${ }^{(\dagger)}$ Upper limit. ${ }^{(*)}$ We propose an order of magnitude uncertainty, based on the uncertainties described in our analysis. ${ }^{(*)}$ The HNC abundance was derived assuming $\mathrm{HNC} / \mathrm{H}_{2}=0.01 \times \mathrm{HCN} / \mathrm{H}_{2}$.

References. ${ }^{(1)}$ Schöier et al. (2013), ${ }^{(2)}$ Decin et al. (2010), ${ }^{(3)}$ Schöier et al. (2011), ${ }^{(4)}$ Danilovich et al. (2014), ${ }^{(5)}$ Agúndez et al. (2012), ${ }^{(6)}$ Danilovich et al. (2018), ${ }^{(7)}$ Brunner et al. (2018), ${ }^{(8)}$ Massalkhi et al. (2019), ${ }^{(9)}$ De Beck \& Olofsson (2018), ${ }^{(10)}$ González Delgado et al. (2003), ${ }^{(11)}$ Ramstedt et al. (2009), ${ }^{(12)}$ Schöier et al. (2006), ${ }^{(13)}$ Maercker et al. (2016), ${ }^{(14)}$ Wong et al. (2018), ${ }^{(15)}$ Schmidt et al. (2016), ${ }^{(16)}$ Agúndez (2009), ${ }^{(17)}$ Massalkhi et al. (2018), ${ }^{(18)}$ De Beck et al. (2012), ${ }^{(19)}$ Velilla Prieto et al. (2017), ${ }^{(20)}$ Daniel et al. (2012), ${ }^{(21)}$ Danilovich et al. (2017), ${ }^{(22)}$ De Beck et al. (2013), ${ }^{(23)}$ Pulliam et al. (2011), ${ }^{(24)}$ Danilovich et al. (2016). 